ANALYSIS \& PDE Volume $2 \quad$ No. $3 \quad 2009$

OLIVIER DRUET AND EMMANUEL HEBEY

STABILITY FOR STRONGLY COUPLED CRITICAL ELLIPTIC SYSTEMS

IN A FULLY INHOMOGENEOUS MEDIUM

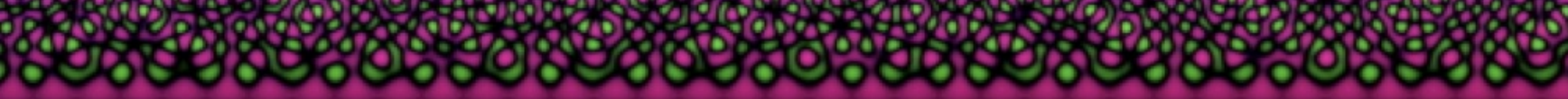

mathematical sciences publishers 


\title{
STABILITY FOR STRONGLY COUPLED CRITICAL ELLIPTIC SYSTEMS IN A FULLY INHOMOGENEOUS MEDIUM
}

\author{
Olivier DRUet AND EMmANUEl HeBEy
}

We investigate and prove analytic stability for strongly coupled critical elliptic systems in the inhomogeneous context of a compact Riemannian manifold.

Coupled systems of nonlinear Schrödinger equations are now a part of several important branches of mathematical physics. They appear in the Hartree-Fock theory for Bose-Einstein double condensates, in fiber-optic theory, in the theory of Langmuir waves in plasma physics, and in the behavior of deep water waves and freak waves in the ocean. A general reference book on such systems and their role in physics has been written by Ablowitz et al. [2004]. We focus here on coupled Gross-Pitaevskii type equations. These systems of equations are strongly related to two branches of mathematical physics. They arise [Burke et al. 1997] in the Hartree-Fock theory for double condensates, which are binary mixtures of Bose-Einstein condensates in two different hyperfine states. They also arise in the study of incoherent solitons in nonlinear optics, as describe in [Akhmediev and Ankiewicz 1998; Christodoulides et al. 1997; Hioe 1999; Hioe and Salter 2002; Kanna and Lakshmanan 2001]. Looking for standing wave solutions for these time evolution systems gives rise to their elliptic analogues that we investigate here. We consider these elliptic systems of equations in arbitrary dimensions $n \geq 3$, in the critical energy regime, and in a fully inhomogeneous medium that we model by an arbitrary compact Riemannian manifold, thus breaking the various symmetries that these systems enjoy in the Euclidean setting.

In what follows we let $(M, g)$ be a smooth compact Riemannian manifold of dimension $n \geq 3$. For $p \geq 1$ an integer, we let $M_{p}^{s}(\mathbb{R})$ denote the vector space of symmetrical $p \times p$ real matrices, and $A$ be a $C^{1}$ map from $M$ to $M_{p}^{s}(\mathbb{R})$. We can write $A=\left(A_{i j}\right)_{i, j}$, where the $A_{i j}$ 's are $C^{1}$ real valued functions in $M$. Let $\Delta_{g}=-\operatorname{div}_{g} \nabla$ be the Laplace-Beltrami operator on $M$. Let also $H^{1}(M)$ be the Sobolev space of functions in $L^{2}(M)$ with one derivative in $L^{2}(M)$. A $p$-map $U=\left(u_{1}, \ldots, u_{p}\right)$ from $M$ to $\mathbb{R}^{p}$ is said to be nonnegative if $u_{i} \geq 0$ for all $i$. The coupled system of nonlinear Schrödinger equations we consider here is written as

$$
\Delta_{g} u_{i}+\sum_{j=1}^{p} A_{i j}(x) u_{j}=|\mathcal{u}|^{2^{\star}-2} u_{i}
$$

in $M$ for all $i$, where $|u|^{2}=\sum_{i=1}^{p} u_{i}^{2}$, and $2^{\star}=2 n /(n-2)$ is the critical Sobolev exponent for the embeddings of the Sobolev space $H^{1}(M)$ into Lebesgue's spaces. The systems (0-1) are weakly coupled by the linear matrix $A$, and strongly coupled by the Gross-Pitaevskii type nonlinearity in the right hand side of (0-1). Besides, (0-1) is critical for Sobolev embeddings. From the viewpoint of conformal

MSC2000: primary 35C20, 58J37; secondary 35Q51, 35Q55, 35Q60.

Keywords: Critical equations, elliptic systems, Riemannian manifolds, stability, strong coupling.

The authors were partially supported by the ANR grant ANR-08-BLAN-0335-01. 
geometry, our systems are pure extensions of Yamabe type equations in the strongly coupled regime. As a by-product (0-1) inherits a conformal structure.

Our aim in this paper is to discuss stability for systems like (0-1). Contrary to time evolution equations, where perturbations of the initial data together with perturbations of the equations are used to measure stability, stability for elliptic equations has to do solely with perturbations of the equations. In the framework of systems such as (0-1), stability is naturally measured with respect to perturbations of the map $A$. In what follows, a system like (0-1) is said to be analytically stable if for any sequence $\left(A_{\alpha}\right)_{\alpha}$ of maps from $M$ to $M_{p}^{s}(\mathbb{R}), \alpha \in \mathbb{N}$, and for any bounded sequence in $H^{1}$ of nonnegative nontrivial solutions $u_{\alpha}$ of the associated systems (0-1), if $A_{\alpha} \rightarrow A$ in $C^{1}$ as $\alpha \rightarrow+\infty$, then, up to a subsequence, $u_{\alpha} \rightarrow U$ in $C^{2}$ as $\alpha \rightarrow+\infty$ for some nonnegative nontrivial solution $u$ of (0-1). When the strong convergence in $C^{2}$ is replaced by a weak convergence $u_{\alpha} \rightarrow U$ in $H^{1}$, the system (0-1) is said to be weakly stable. We refer to Section 1 for more precise definitions.

Before stating our theorem we need to introduce two assumptions. Let $\boldsymbol{\Delta}_{g}$ be the Laplace-Beltrami operator acting on $p$-maps by acting on each of the components of the map, and let $\operatorname{Vect}_{+}\left(\mathbb{R}^{p}\right)$ be the set of vectors in $\mathbb{R}^{p}$ with nonnegative components. The first assumption we may impose is

$$
\operatorname{Ker}\left(\boldsymbol{\Delta}_{g}+A\right) \cap L^{2}\left(M, \operatorname{Vect}_{+}\left(\mathbb{R}^{p}\right)\right)=\{0\},
$$

where $\operatorname{Ker}\left(\boldsymbol{\Delta}_{g}+A\right)$ is the kernel of $\boldsymbol{\Delta}_{g}+A$, and $L^{2}\left(M, \operatorname{Vect}+\left(\mathbb{R}^{p}\right)\right)$ stands for the set of $L^{2}$ maps from $M$ to $\operatorname{Vect}_{+}\left(\mathbb{R}^{p}\right)$. In order to introduce our second assumption we let $A_{n}=A_{n}(A)$ be given by

$$
A_{n}=A-\frac{n-2}{4(n-1)} S_{g} \operatorname{Id}_{p},
$$

where $S_{g}$ is the scalar curvature of $g$, and $\operatorname{Id}_{p}$ is the identity $p \times p$ matrix. For $x \in M$, let also $\operatorname{Is}_{A_{n}(x)}$ be the set consisting of the isotropic vectors for $A_{n}(x)$, namely of the vectors $X \in \mathbb{R}^{p}$ which are such that $\left\langle A_{n}(x) . X, X\right\rangle_{\mathbb{R}^{p}}=0$, where $\langle\cdot, \cdot\rangle_{\mathbb{R}^{p}}$ is the Euclidean scalar product in $\mathbb{R}^{p}$. The second assumption we introduce is that for any $x \in M, A_{n}(x)$ should not possess stable subspaces with an orthonormal basis consisting of isotropic nonnegative vectors. More precisely, it is this:

For any $x \in M$ and any $k \in\{1, \ldots, p\}$, there does not exist an orthonormal family $\left(e_{1}, \ldots, e_{k}\right)$ of vectors in $\operatorname{Is}_{A_{n}(x)} \cap \operatorname{Vect}_{+}\left(\mathbb{R}^{p}\right)$ such that $A_{n}(x) V \subset V$, where $V$ is the $k$-dimensional subspace of $\mathbb{R}^{p}$ with basis $\left(e_{1}, \ldots, e_{k}\right)$.

The case $k=1$ in $\left(\mathrm{H}^{\prime}\right)$ reduces to the nonexistence of a nontrivial vector in $\operatorname{Vect}_{+}\left(\mathbb{R}^{p}\right) \cap \operatorname{Ker} A_{n}(x)$, where $\operatorname{Ker} A_{n}(x)$ is the kernel of $A_{n}(x)$. An assumption like $\left(\mathrm{H}^{\prime}\right)$ is automatically satisfied in several simple situations. This is the case if we prevent the existence of isotropic vectors for $A_{n}$. In particular, $\left(\mathrm{H}^{\prime}\right)$ holds true if $A_{n}(x)>0$ or $A_{n}(x)<0$ for all $x$ in the sense of bilinear forms. Clearly there are other cases where $\left(\mathrm{H}^{\prime}\right)$ holds true. Assumption $(\mathrm{H})$ is analytic in nature. Assumption $\left(\mathrm{H}^{\prime}\right)$ is algebraic in nature and related to the underlying geometric conformal structure of the equations. Our main result, establishing analytic stability for (0-1), is stated as follows.

Theorem 0.1. Let $(M, g)$ be a smooth compact Riemannian manifold of dimension $n \geq 4$ and $p \geq 1$ be an integer. For any $C^{1}$-map $A: M \rightarrow M_{p}^{s}(\mathbb{R})$ satisfying $(\mathrm{H})$ and $\left(\mathrm{H}^{\prime}\right)$, the system $(0-1)$ is analytically stable when $n \neq 6$, and weakly stable when $n=6$. Besides, there are examples of six-dimensional manifolds and $C^{1}$-maps A satisfying $(\mathrm{H})$ and $\left(\mathrm{H}^{\prime}\right)$ for which $(0-1)$ is analytically unstable. 
A closely related notion to stability is that of compactness. A system like (0-1) is said to be compact if bounded sequences in $H^{1}$ of nonnegative solutions of (0-1) converge, up to a subsequence, in the $C^{2}$ topology. As is easily seen, analytic stability implies compactness. In particular, as a direct consequence of the analytic stability part in our theorem, we get that systems like (0-1) are compact when $n \neq 6$ as soon as $(\mathrm{H})$ and $\left(\mathrm{H}^{\prime}\right)$ are satisfied. Assumptions $(\mathrm{H})$ and $\left(\mathrm{H}^{\prime}\right)$ in Theorem 0.1 are sharp, as discussed in Section 1 below.

Most of the asymptotic analysis developed in this paper carries over to the $n=3$ case. However, the concluding argument needs to be changed when $n=3$. In this dimension the mass of the Green's expansion of the Schrödinger operator $\Delta_{g}+A$ leads over $A_{n}$. We can conclude when the mass is positive. The analysis is developed in [Druet et al. 2009].

The paper is organized as follows. We discuss general properties of stability and compactness in Section 1. We prove the $n=6$ part of Theorem 0.1 in Section 2. We provide a complete classification of $H^{1}$-nonnegative solutions of the strongly coupled critical limit Euclidean system $\Delta u_{i}=|u|^{2^{\star}-2} u_{i}$, $i=1, \ldots, p$, in Section 3. We prove Theorem 0.1 in its $n \neq 6$ part in Sections 4 to 10. In the process we establish in Sections 5, 6, and 8 the full $C^{0}$-theory for the blow-up of arbitrary sequences of solutions of strongly coupled systems like (0-1).

\section{General considerations on stability and compactness}

We start with the precise definition of elliptic stability we use for our systems (0-1). As already mentioned stability is here measured with respect to perturbations of the parameter $A$ in (0-1). In doing so we preserve the conformal structure of the equation. Historically speaking such type of perturbations were first considered in the early work of Aubin [1976] on the Yamabe equation. Given $\left(A_{\alpha}\right)_{\alpha}$ a sequence of $C^{1}$ maps from $M$ to $M_{p}^{s}(\mathbb{R})$, with $A_{\alpha}=\left(A_{i j}^{\alpha}\right)_{i, j}$ for all $\alpha$ integer, we consider the systems

$$
\Delta_{g} u_{i}+\sum_{j=1}^{p} A_{i j}^{\alpha}(x) u_{j}=|u|^{2^{\star}-2} u_{i} .
$$

A sequence $\left(U_{\alpha}\right)_{\alpha}$ of $C^{2}$ maps from $M$ to $\mathbb{R}^{p}$ is said to be a sequence of nonnegative solutions of (1-1) if for any $\alpha \in \mathbb{N}, u_{\alpha}=\left(u_{1}^{\alpha}, \ldots, u_{p}^{\alpha}\right)$ solves (0-1) and $u_{i}^{\alpha} \geq 0$ for all $i$. The sequence is said to be bounded in $H^{1}(M)$, or to have finite energy, if its components $u_{\alpha}^{i}$ are all bounded in $H^{1}(M)$ with respect to $\alpha$. Given $\Lambda>0$, we define the slice $\mathscr{S}_{A}^{\Lambda}$ to be the set of $p$-maps $U \in H^{1}$ such that $U$ solves (0-1), $U$ is nonnegative and the $H^{1}$-norm of $U$ is less than or equal to $\Lambda$. By standard regularity, adapting classical arguments from Trudinger [1968], weak solutions in $H^{1}$ of systems like (0-1) are always of class $C^{2}$. In particular, $\mathscr{Y}_{A}^{\Lambda} \subset C^{2}$ for all $\Lambda>0$. For $X, Y \subset C^{2}$ we let $d_{C^{2}}^{\hookrightarrow}(X ; Y)$ be the $C^{2}$-pointed distance from $X$ to $Y$ defined by

$$
d_{C^{2}}^{\hookrightarrow}(X ; Y)=\sup _{U \in X} \inf _{\mathscr{V} \in Y}\|\mathscr{V}-U\|_{C^{2}},
$$

where $\|\mathscr{V}-\mathscr{U}\|_{C^{2}}=\sum_{i}\left\|v_{i}-u_{i}\right\|_{C^{2}}$ and the $u_{i}$ 's and $v_{i}$ 's are the components of $\mathcal{U}$ and $\mathscr{V}$. Stability in the elliptic regime is defined in Definition 1.1 below. The $C^{1}$ convergence $A_{\alpha} \rightarrow A$ in Definition 1.1 refers to the $C^{1}$ convergence of the components $A_{i j}^{\alpha}$ of $A_{\alpha}$ to the components $A_{i j}$ of $A$. Similarly, the $C^{2}$ convergences, and the weak convergences in $H^{1}$, of the $U_{\alpha}$ 's in Definition 1.1 refer to the convergences of the components of the maps. 
Definition 1.1. Let $(M, g)$ be a smooth compact Riemannian manifold, let $p \geq 1$ be an integer, and let $A: M \rightarrow M_{p}^{s}(\mathbb{R})$ be a $C^{1}$ map. The system (0-1) is said to be

(i) analytically stable if for any sequence $\left(A_{\alpha}\right)_{\alpha}$ of $C^{1}$ maps from $M$ to $M_{p}^{s}(\mathbb{R})$ such that $A_{\alpha} \rightarrow A$ in $C^{1}(M)$ as $\alpha \rightarrow+\infty$, and for any bounded sequence $\left(\vartheta_{\alpha}\right)_{\alpha}$ in $H^{1}(M)$ of nonnegative nontrivial solutions of (1-1), there exists a nonnegative nontrivial solution $\mathcal{U}$ of $(0-1)$ such that, up to a subsequence, the $u_{\alpha}$ 's converge strongly to $u$ in $C^{2}(M)$ as $\alpha \rightarrow+\infty$, and

(ii) weakly stable if for any sequence $\left(A_{\alpha}\right)_{\alpha}$ of $C^{1}$ maps from $M$ to $M_{p}^{s}(\mathbb{R})$ such that $A_{\alpha} \rightarrow A$ in $C^{1}(M)$ as $\alpha \rightarrow+\infty$ and for any bounded sequence $\left(U_{\alpha}\right)_{\alpha}$ in $H^{1}(M)$ of nonnegative nontrivial solutions of (1-1), there exists a nonnegative nontrivial solution $U$ of $(0-1)$ such that, up to a subsequence, the $u_{\alpha}$ 's converge weakly to $u$ in $H^{1}(M)$ as $\alpha \rightarrow+\infty$.

The system is said to be geometrically stable, if the slices $\mathscr{S}_{A}^{\Lambda}$ are stable for all $\Lambda>0$, where $\mathscr{S}_{A}^{\Lambda}$ is said to be stable, if for any $\varepsilon>0$, there exists $\delta>0$ such that for any $C^{1}$ map $A^{\prime}$ from $M$ to $M_{p}^{s}(\mathbb{R})$, we have $d_{C^{2}}^{\hookrightarrow}\left(\mathscr{Y}_{A^{\prime}}^{\Lambda} ; \mathscr{Y}_{A}^{\Lambda}\right)<\varepsilon$ when $\left\|A^{\prime}-A\right\|_{C^{1}}<\delta$.

As already mentioned, a classical notion in the study of critical elliptic equations is that of compactness. A system like (0-1) is said to be compact if any bounded sequence $\left(\vartheta_{\alpha}\right)_{\alpha}$ in $H^{1}(M)$ of nonnegative nontrivial solutions of (0-1) converges in $C^{2}(M)$ as $\alpha \rightarrow+\infty$ to a nonnegative nontrivial solution $U$ of $(0-1)$. This corresponds to the particular situation where $A_{\alpha}=A$ for all $\alpha$ in (i). Analytic stability as defined in (i) implies weak stability, geometric stability, and compactness. More precisely:

Proposition 1.2. Assume (H). If the system (0-1) is analytically stable, it is weakly stable, geometrically stable, and compact. A compact system is analytically stable if and only if it is geometrically stable.

Proof. It is obvious that analytic stability implies weak stability, geometric stability, and compactness. The only assertion, which deserves to be proved, is that a compact geometrically stable system like (0-1) is analytically stable. Let $\left(A_{\alpha}\right)_{\alpha}$ be a sequence of $C^{1}$ maps from $M$ to $M_{p}^{s}(\mathbb{R})$ such that $A_{\alpha} \rightarrow A$ in $C^{1}(M)$ as $\alpha \rightarrow+\infty$, and let $\left(U_{\alpha}\right)_{\alpha}$ be a bounded sequence in $H^{1}$ of nonnegative nontrivial solutions of (1-1). Since (0-1) is geometrically stable there exists $\left(\mathscr{V}_{\alpha}\right)_{\alpha}$, a bounded sequence in $H^{1}$ of nonnegative solutions of (0-1), such that, up to a subsequence, $\mathcal{U}_{\alpha}-\mathscr{V}_{\alpha}$ converges to zero in $C^{2}$ as $\alpha \rightarrow+\infty$. Since (0-1) is compact, up to a subsequence, $\mathscr{V}_{\alpha} \rightarrow \mathscr{V}$ in $C^{2}$ as $\alpha \rightarrow+\infty$, where $\mathscr{V}$ is a nonnegative solution of (0-1). In particular, up to a subsequence, $\mathcal{U}_{\alpha} \rightarrow \mathscr{V}$ in $C^{2}$ as $\alpha \rightarrow+\infty$. It remains to prove that $\mathscr{V}$ is nontrivial, and this is given by Lemma 1.3 below. Proposition 1.2 is proved.

The following lemma, which we derive as a direct consequence of $(\mathrm{H})$, was used in the proof of Proposition 1.2. By standard elliptic theory, moreover, when $A$ satisfies (H), we have $u_{\alpha} \not \nrightarrow 0$ in $H^{1}$ as $\alpha \rightarrow+\infty$.

Lemma 1.3. Let $(M, g)$ be a smooth compact Riemannian manifold, let $p \geq 1$ be an integer, and let $A: M \rightarrow M_{p}^{s}(\mathbb{R})$ be a $C^{1}$ map satisfying $(\mathrm{H})$. Let $\left(A_{\alpha}\right)_{\alpha}$ be a sequence of $C^{1}$ maps from $M$ to $M_{p}^{s}(\mathbb{R})$ such that $A_{\alpha} \rightarrow A$ in $C^{1}(M)$ as $\alpha \rightarrow+\infty$, and let $\left(\cup_{\alpha}\right)_{\alpha}$ be a bounded sequence in $H^{1}$ of nonnegative nontrivial solutions of (1-1). Then $u_{\alpha} \not>0$ in $L^{\infty}(M)$ as $\alpha \rightarrow+\infty$.

Proof. By contradiction we assume that there exists $\left(u_{\alpha}\right)_{\alpha}$, a bounded sequence in $H^{1}$ of nonnegative nontrivial solutions of (1-1), such that $\max _{M}\left|u_{\alpha}\right|_{\Sigma} \rightarrow 0$ as $\alpha \rightarrow+\infty$, where $\left|u_{\alpha}\right|_{\Sigma}=\sum_{i} u_{i, \alpha}$ is the sum of the components of the $u_{\alpha}$ 's. Let $\varepsilon_{\alpha}=\left|u_{\alpha}\right|_{\Sigma}$ and define $v_{i, \alpha}$ by $v_{i, \alpha}=\varepsilon_{\alpha}^{-1} u_{i, \alpha}$ for all $i$ and $\alpha$. Then 


$$
\Delta_{g} v_{i, \alpha}+\sum_{j=1}^{p} A_{i j}^{\alpha}(x) v_{j, \alpha}=\varepsilon_{\alpha}^{4 /(n-2)}\left|\mathscr{V}_{\alpha}\right|^{2^{\star}-2} v_{i, \alpha}
$$

for all $i$ and $\alpha$, where $\mathscr{V}_{\alpha}$ is the $p$-map whose components are the $v_{i, \alpha}$ 's for $i=1, \ldots, p$. By construction the $v_{i, \alpha}$ 's are bounded in $L^{\infty}(M)$. By standard elliptic theory it follows that, up to a subsequence, they converge in $C^{2}(M)$ to $v_{i}$ 's as $\alpha \rightarrow+\infty$. Let $\mathscr{V}$ be the $p$-map with components $v_{i}$ for $i=1, \ldots, p$. By construction $\mathscr{V}$ is nonnegative and nontrivial, since there is one point, where $|\mathscr{V}|_{\Sigma}$ equals one. Letting $\alpha \rightarrow+\infty$ in (1-3) it follows that $\mathscr{V} \in \operatorname{Ker}\left(\boldsymbol{\Delta}_{g}+A\right)$, and we get a contradiction with (H).

Proposition 1.2 leaves open the question of whether or not there exist geometrically stable noncompact systems like (0-1). However, we can have noncompact systems with geometrically stable specific slices as discussed below. The most well-known example of a noncompact critical system like (0-1) is given by the Yamabe equation on the sphere. The Yamabe equation on the $n$-sphere possesses a $(n+1)$-parameter noncompact family of solutions and it turns out that it is also geometrically unstable. This is a direct consequence of the constructions in [Druet and Hebey 2005a], where arbitrarily high energy solutions of approximated equations are constructed, together with the property that all nonnegative nontrivial solutions of the Yamabe equation on the sphere have the same energy. On the other hand, the first blowup slice for this equation is geometrically $H^{1}$-stable in the sense of Definition 1.1 when we replace the $C^{2}$-pointed distance and the $C^{2}$-norm in (1-2) by a $H^{1}$-pointed distance and a $H^{1}$-norm, where the first blow-up slice is given by $\Lambda=K_{n}^{2}$, and $K_{n}$ is as in (3-8). This geometric $H^{1}$-stability of the first blowup slice follows from $H^{1}$-decompositions as in Proposition 4.2. As a direct consequence, noncompact equations may have stable slices.

In the subcritical regime, compactness goes back to [Gidas and Spruck 1981]. In the more involved critical regime, it goes back to Schoen's conjecture [Schoen 1989; 1991] that compactness holds true for the geometric Yamabe equation as soon as the background manifold is distinct from the sphere. His conjecture has been a source of motivations for several years. The conjecture was proved to be true for conformally flat manifolds by Schoen [1989; 1991]. The nonconformally flat case turned out to be more intricate. The case of low-dimensional manifolds was recently addressed in [Druet 2004; Marques 2005; Li and Zhu 1999; Li and Zhang 2004; 2005], and compactness up to dimension 24 was finally proved recently [Khuri et al. 2009]. On the other hand, Brendle [2008a] and Brendle and Marques [2009] exhibited counterexamples to the conjecture in dimensions $n \geq 25$. For any $n \geq 25$ they constructed examples of nonconformally flat $n$-manifolds with the striking property that their associated Yamabe equations possess sequences of solutions with minimal type energy and unbounded $L^{\infty}$-norms. In particular, they proved the very surprising result that the compactness conjecture is false for nonconformally flat manifolds in any dimension $n \geq 25$. A very interesting survey on the subject is [Brendle 2008b]. We refer also to [Druet and Hebey 2005b].

An easy remark is that if $u$ is a solution of a scalar Yamabe type equation with linear term $h$, that is, an equation of the form

$$
\Delta_{g} u+h(x) u=u^{2^{\star}-1},
$$

then $u=\left(\frac{1}{\sqrt{p}} u, \ldots, \frac{1}{\sqrt{p}} u\right)$ is a solution of (0-1) when $A_{i j}=h a_{i j}$ for all $i, j$, and $\sum_{j=1}^{p} a_{i j}=1$ for all $i$. In what follows we let $\left(a_{i j}\right)_{i, j}$ be a symmetrical matrix of $C^{1}$ functions $a_{i j}: M \rightarrow \mathbb{R}$ such that $\sum_{j=1}^{p} a_{i j}(x)=1$ for all $i=1, \ldots, p$ and all $x \in M$. A possible choice is $a_{i j}=\delta_{i j}$ for all $i, j$. Then we 
define $A(g)$ and $A^{\prime}(g)$ to be the $C^{1}$ maps from $M$ to $M_{p}^{s}(\mathbb{R})$ given by

$$
A(g)_{i j}=\frac{n-2}{4(n-1)} S_{g} a_{i j} \quad \text { and } \quad A^{\prime}(g)_{i j}=\frac{n-2}{4(n-1)}\left(\max _{M} S_{g}\right) a_{i j}
$$

for all $i, j=1, \ldots, p$, where $S_{g}$ is the scalar curvature of $g$. By combining results in [Brendle 2008a; Brendle and Marques 2009], where noncompactness of the Yamabe equation in the nonconformally flat case is investigated, and those in [Druet and Hebey 2005a; Hebey and Vaugon 2001], where unstability of Yamabe type equations in the conformally flat case is investigated, we obtain the following theorem, in view of the remark above.

Theorem 1.4. The system (0-1) associated with $A(g)$ is analytically unstable when posed on spherical spaces forms in any dimension $n \geq 6$, and even noncompact when posed on the sphere in any dimension $n \geq 3$. For any conformally flat manifold $(M, g)$ of dimension $n \geq 4$ there exists a conformal metric $\tilde{g}$ to $g$ of nonconstant scalar curvature having one and only one maximum point such that the system (0-1) associated with $A^{\prime}(\tilde{g})$ is analytically unstable. In any dimension $n \geq 25$ there are examples of nonconformally flat manifolds such that the system (0-1) associated with A(g) is noncompact, and thus also analytically unstable.

The examples in Theorem 1.4 do not satisfy $\left(\mathrm{H}^{\prime}\right)$. This can be checked by noting that $(1, \ldots, 1) \in$ $\operatorname{Ker} A_{n}(x)$ for all $x$, where $A_{n}$ is as in (0-2). Theorem 0.1 and Theorem 1.4 complement each other. As a remark, the Yamabe equation on quotients of the sphere is obviously compact since it possesses a unique solution. In particular, there are compact equations which are neither analytically nor geometrically stable. Compactness does not imply stability. We concentrate in the rest of this section on the subcritical regime for systems like (0-1) and prove that analytic stability holds true in the subcritical regime without assuming $\left(\mathrm{H}^{\prime}\right)$. Let $q \in\left(2,2^{\star}\right)$ and let us consider the subcritical system

$$
\Delta_{g} u_{i}+\sum_{j=1}^{p} A_{i j}(x) u_{j}=|q|^{q-2} u_{i}
$$

in $M$ for all $i$, where $A=\left(A_{i j}\right)_{i, j}$ is a $C^{1}$ map from $M$ to $M_{p}^{s}(\mathbb{R})$. We define the notions of analytic stability, weak stability, and geometric stability for (1-5) as in Definition 1.1.

Proposition 1.5. Let $(M, g)$ be a smooth compact Riemannian manifold of dimension $n \geq 3, p \geq 1$ be an integer, and $A: M \rightarrow M_{p}^{s}(\mathbb{R})$ be a $C^{1}$ map satisfying $(\mathrm{H})$. For any $q \in\left(2,2^{\star}\right)$ the subcritical system (1-5) is analytically stable.

Proof. Let $\left(A_{\alpha}\right)_{\alpha}$ be a sequence of $C^{1}$ maps from $M$ to $M_{p}^{s}(\mathbb{R})$ such that $A_{\alpha} \rightarrow A$ in $C^{1}(M)$ as $\alpha \rightarrow+\infty$, and let $\left(u_{\alpha}\right)_{\alpha}$ be an arbitrary bounded sequence in $H^{1}$ of nonnegative nontrivial solutions of

$$
\Delta_{g} u_{i, \alpha}+\sum_{j=1}^{p} A_{i j}^{\alpha}(x) u_{j, \alpha}=\left|u_{\alpha}\right|^{q-2} u_{i, \alpha}
$$

for all $i$ and all $\alpha$. We aim in proving that a subsequence of $\left(u_{\alpha}\right)_{\alpha}$ converges in $C^{2}$ to a nonnegative nontrivial solution of (1-6). The nontriviality of any strong limit follows from $(\mathrm{H})$ mimicking the proof of Lemma 1.3. Then, as is easily checked, it suffices to prove that the $u_{\alpha}$ 's are $L^{\infty}$-bounded in $M$. By contradiction we assume that there exists a sequence $\left(x_{\alpha}\right)_{\alpha}$ of points, where the $\left|u_{\alpha}\right|$ 's are maximum 
such that, up to a subsequence, $\left|u_{\alpha}\left(x_{\alpha}\right)\right| \rightarrow+\infty$ as $\alpha \rightarrow+\infty$. Let $\mu_{\alpha}=\left|u_{\alpha}\left(x_{\alpha}\right)\right|^{-(q-2) / 2}$. Then $\mu_{\alpha} \rightarrow 0$ as $\alpha \rightarrow+\infty$. Let $\tilde{u}_{\alpha}$ be given for $x \in \mathbb{R}^{n}$ by

$$
\tilde{u}_{\alpha}(x)=\mu_{\alpha}^{2 /(q-2)} u_{\alpha}\left(\exp _{x_{\alpha}}\left(\mu_{\alpha} x\right)\right)
$$

and let $g_{\alpha}$ be the metric given by $g_{\alpha}(x)=\left(\exp _{x_{\alpha}}^{\star} g\right)\left(\mu_{\alpha} x\right)$. We have $g_{\alpha} \rightarrow \xi$ in $C_{\text {loc }}^{2}\left(\mathbb{R}^{n}\right)$ as $\alpha \rightarrow+\infty$, where $\xi$ is the Euclidean metric. Noting that

$$
\Delta_{g_{\alpha}} \tilde{u}_{i, \alpha}+\mu_{\alpha}^{2} \sum_{j=1}^{p} A_{i j}^{\alpha}\left(\exp _{x_{\alpha}}\left(\mu_{\alpha} x\right)\right) \tilde{u}_{j, \alpha}=\left|\tilde{u}_{\alpha}\right|^{q-2} \tilde{u}_{i, \alpha}
$$

for all $i$ and $\alpha$, since $\left|\tilde{u}_{\alpha}\right| \leq 1$ for all $\alpha$ by construction, it follows from standard elliptic theory that there exists $\tilde{u} \in C^{2}\left(\mathbb{R}^{n}\right)$ such that, up to a subsequence, $\tilde{U}_{\alpha} \rightarrow \tilde{u}$ in $C_{\text {loc }}^{2}\left(\mathbb{R}^{n}\right)$. We have $\left|\tilde{U}_{\alpha}(0)\right|=1$ for all $\alpha$. Hence, $|\tilde{u}(0)|=1$. Moreover, for any $R>0$, and for $\alpha$ sufficiently large,

$$
\begin{aligned}
\int_{B_{0}(R)}|\tilde{u}|^{q} d x & \leq C \int_{B_{0}(R)}\left|\tilde{u}_{\alpha}\right|^{q} d v_{g_{\alpha}} \\
& \leq C \int_{B_{0}\left(1 / \mu_{\alpha}\right)}\left|\tilde{u}_{\alpha}\right|^{q} d v_{g_{\alpha}}=C \mu_{\alpha}^{2 q /(q-2)-n} \int_{B_{x_{\alpha}}(1)}\left|u_{\alpha}\right|^{q} d v_{g} \leq C \mu_{\alpha}^{2 q /(q-2)-n},
\end{aligned}
$$

since the $\boldsymbol{U}_{\alpha}$ 's have bounded energy. Noting that $2 q /(q-2)>n$ as soon as $q<2^{\star}$ and letting $\alpha \rightarrow+\infty$ in the inequality above, we get $\int_{B_{0}(R)}|\tilde{u}|^{q} d x=0$. This is in contradiction with $|\tilde{u}(0)|=1$. The proposition is proved.

Analytic stability for critical equations like (1-4) has been investigated in [Druet 2003]. The case $p=1$ in Theorem 0.1 , in its $n \neq 6$ part and when considering $C^{2, \theta}$-perturbations of $h$, was proved in the same paper. The proof we propose here extends to the case of systems, allows us to consider $C^{0, \eta_{-}}$ perturbations of $h$, see the remark at the end of Section 10, and is more direct. At the time of [Druet 2003], analytic stability was still referred to as compactness. The confusion in the terminology has been the source of several misunderstandings.

\section{The six-dimensional case}

We discuss and prove the six-dimensional last assertion in Theorem 0.1 concerning the existence of systems like (0-1) in dimension $n=6$, which satisfy $(\mathrm{H})$ and $\left(\mathrm{H}^{\prime}\right)$, but which, contrary to what happens when $n \neq 6$, are not analytically stable. We restrict ourselves to a very explicit construction in the case of the unit sphere $\left(S^{6}, g_{0}\right)$. A more general discussion could have been developed. We let $\left(a_{i j}\right)_{i, j}$ be a symmetrical matrix of $C^{1}$ functions $a_{i j}: S^{6} \rightarrow \mathbb{R}$ such that $\sum_{j=1}^{p} a_{i j}(x)=1$ for all $i=1, \ldots, p$ and all $x \in S^{6}$. If $h: S^{6} \rightarrow \mathbb{R}$ is of class $C^{1}$, we define $A(h)$ to be the $C^{1}$ map from $S^{6}$ to $M_{p}^{s}(\mathbb{R})$ with components $A(h)_{i j}$ given by $A(h)_{i j}=h a_{i j}$ for all $i, j=1, \ldots, p$. When $n=6$, we have $2^{\star}=3$. For the unit sphere $\left(S^{6}, g_{0}\right)$, we also have

$$
\frac{n-2}{4(n-1)} S_{g_{0}}=6 \text {. }
$$

Proposition 2.1. Let $\left(S^{6}, g_{0}\right)$ be the unit six-dimensional sphere in $\mathbb{R}^{7}$. There exists $h: S^{6} \rightarrow \mathbb{R}, h>6$ everywhere and of class $C^{1}$, such that the system (0-1) associated with $A=A(h)$ is analytically unstable. 
Proof. We fix $x_{0} \in S^{6}$ and let $r$ be the distance to $x_{0}$. We let also $\theta$ be given by $\theta=\cos r$. First we claim that there exist smooth positive functions $h$ and $u$ in $S^{6}$, which we write into the form $h(x)=\hat{h}(\theta)$ and $u(x)=\hat{u}(\theta)$, such that

$$
\Delta_{g_{0}} u+h u=u^{2} \quad \text { and } \quad h>6
$$

in $S^{6}$, and such that

$$
\hat{h}(1)=3 \hat{u}(1), \quad \hat{u}(1)=6 \quad \text { and } \quad \hat{h}^{\prime}(1)=2 \hat{u}^{\prime}(1) .
$$

To prove the claim we let $\hat{u}$ be given by

$$
\hat{u}(\theta)=6\left(1-2(\theta-1)+3(\theta-1)^{2}\right) .
$$

Clearly, $\hat{u}(1)=6$ and $\hat{u}^{\prime}(1)=-12$. Since $\Delta_{g_{0}} \theta=6 \theta$ and $|\nabla \theta|^{2}=1-\theta^{2}$, we get

$$
\frac{1}{6} \Delta_{g_{0}} u=6\left(7 \theta^{2}-8 \theta-1\right) .
$$

In particular, the first equation in (2-1) is satisfied if we let $\hat{h}$ be given by

$$
\frac{1}{6} \hat{h}(\theta)=3 \theta^{2}-8 \theta+6-\frac{7 \theta^{2}-8 \theta-1}{3 \theta^{2}-8 \theta+6} .
$$

As is easily checked from (2-4), $\hat{h}(1)=3 \hat{u}(1)$ and $\hat{h}^{\prime}(1)=2 \hat{u}^{\prime}(1)$. In particular, (2-2) holds true. Noting that $\hat{h}>6$ for all $\theta \in[-1,+1]$, we get two explicit smooth positive functions $h$ and $u$ in $S^{6}$, given by (2-3) and (2-4), such that (2-1) and (2-2) hold true. This proves the above claim. Now, for $\beta>1$, we define $B_{\beta}$ by $B_{\beta}(x)=\hat{B}_{\beta}(\theta)$, where

$$
\hat{B}_{\beta}(\theta)=6\left(\beta^{2}-1\right)(\beta-\theta)^{-2} .
$$

We have $\Delta_{g_{0}} B_{\beta}+6 B_{\beta}=B_{\beta}^{2}$ in $S^{6}$. Let

$$
u_{\beta}=u+B_{\beta}
$$

and $\hat{u}_{\beta}=\hat{u}+\hat{B}_{\beta}$, where $u$ and $\hat{u}$ are as in (2-1) and (2-2). As is easily checked from (2-1) and the equation satisfied by $B_{\beta}$, we have

$$
\Delta_{g_{0}} u_{\beta}+h_{\beta} u_{\beta}=u_{\beta}^{2}
$$

in $S^{6}$ for all $\beta>1$, where $h_{\beta}=\hat{h}_{\beta}(\theta)$ is given by

$$
\hat{h}_{\beta}=\hat{h}-\frac{(12 \hat{u}+6-\hat{h}) \hat{B}_{\beta}}{\hat{u}+\hat{B}_{\beta}} .
$$

Noting, thanks to (2-2), that $h_{\beta} \rightarrow h$ in $C_{\text {loc }}^{0}\left(S^{6}\right)$ as $\beta \rightarrow 1$, while $\hat{h}_{\beta}^{\prime} \rightarrow \hat{h}^{\prime}$ in $L^{\infty}([-1,+1])$ as $\beta \rightarrow 1$, we conclude that $h_{\beta} \rightarrow h$ in $C^{1}\left(S^{6}\right)$. Now we let $\left(\beta_{\alpha}\right)_{\alpha}$ be a sequence of positive real numbers such that $\beta_{\alpha}>1$ for all $\alpha$ and $\beta_{\alpha} \rightarrow 1$ as $\alpha \rightarrow+\infty$. We let $u_{\alpha}=p^{-1 / 2}\left(u_{\beta_{\alpha}}, \ldots, u_{\beta_{\alpha}}\right), A_{\alpha}=A\left(h_{\beta_{\alpha}}\right)$, and $A=A(h)$ where $u_{\beta}$ is given by (2-5), $h_{\beta}=\hat{h}_{\beta}(\theta)$ is given by (2-7), and $h=\hat{h}(\theta)$ is given by (2-4). The $u_{\alpha}$ 's solve (1-1), they have bounded energy, and $A_{\alpha} \rightarrow A$ in $C^{1}$. Noting that $\left\|u_{\alpha}\right\|_{\infty} \rightarrow+\infty$ as $\alpha \rightarrow+\infty$, this proves the proposition.

It is easily checked that $A=A(h)$ satisfies $(\mathrm{H})$. If $U \in L^{2}\left(M, \operatorname{Vect}_{+}\left(\mathbb{R}^{p}\right)\right)$ is in the kernel of the vector Schrödinger operator associated with $A(h)$, we conclude by summing over the components that $|u|_{\Sigma}=\sum_{i=1}^{p} u_{i}$ belongs to $\operatorname{Ker}\left(\Delta_{g_{0}}+h\right)$. This is impossible unless $U \equiv 0$ since $h>0$. It is also easily seen that, at least for small perturbations $a_{i j}$ of $\delta_{i j}$, the map $A=A(h)$ satisfies $\left(\mathrm{H}^{\prime}\right)$. 


\section{The limit system}

Of importance in blow-up theory, when discussing critical equations, is the classification of the solutions of the critical limit Euclidean system we get by blowing up the original equations. In our case, we need to classify the $\dot{H}^{1}$-nonnegative solutions of the limit system

$$
\Delta u_{i}=|u|^{2^{\star}-2} u_{i}
$$

where $|u|^{2}=\sum_{i=1}^{p} u_{i}^{2}$, and $\Delta=-\sum_{i=1}^{n} \partial^{2} / \partial x_{i}^{2}$ is the Euclidean Laplace-Beltrami operator . Depending on the context, we let $\dot{H}^{1}\left(\mathbb{R}^{n}\right)$ be the homogeneous Sobolev space defined as the completion of functions with compact supports, or of $p$-maps with compact supports, with respect to the $L^{2}$-norm of their gradient. The classification result we prove here is stated as follows.

Proposition 3.1. Let $p \geq 1$ and $U \in \dot{H}^{1}\left(\mathbb{R}^{n}\right)$ be a nonnegative solution of (3-1). Then there exist $a \in \mathbb{R}^{n}$, $\lambda>0$, and $\Lambda \in S_{+}^{p-1}$, such that

$$
u(x)=\left(\frac{\lambda}{\lambda^{2}+\frac{|x-a|^{2}}{n(n-2)}}\right)^{(n-2) / 2} \Lambda
$$

for all $x \in \mathbb{R}^{n}$, where $S_{+}^{p-1}$ consists of those elements $\left(\Lambda_{1}, \ldots, \Lambda_{p}\right)$ in the unit sphere $S^{p-1}$ (in $\left.\mathbb{R}^{p}\right)$ that satisfy $\Lambda_{i} \geq 0$ for all $i$.

We prove Proposition 3.1 in several steps. Let $u \in \dot{H}^{1}\left(\mathbb{R}^{n}\right)$ be a nonnegative solution of (3-1). Regularity theory and the maximum principle apply to (3-1). In particular, $U$ is necessarily smooth with the property that for any $i$, either $u_{i} \equiv 0$ or $u_{i}>0$ in $\mathbb{R}^{n}$. We may therefore assume that there exists $p^{\prime} \leq p$ such that $u_{i}>0$ in $\mathbb{R}^{n}$ for all $i=1, \ldots, p^{\prime}$. A first step in the proof of Proposition 3.1 is as follows.

Step 1. Let $u \in \dot{H}^{1}\left(\mathbb{R}^{n}\right)$ be a nonnegative solution of (3-1) such that $u_{i}>0$ in $\mathbb{R}^{n}$ for all $i=1, \ldots, p^{\prime}$, $p^{\prime} \leq p$. Then, for any $R>0$,

$$
\min _{\partial B_{0}(R)} \frac{u_{i}}{u_{j}} \leq \frac{u_{i}}{u_{j}} \leq \max _{\partial B_{0}(R)} \frac{u_{i}}{u_{j}}
$$

in $B_{0}(R)$ for all $i, j \in\left\{1, \ldots, p^{\prime}\right\}$.

Proof of Step 1. By (3-1),

$$
\Delta\left(\frac{u_{i}}{u_{j}}\right)=2\left(\nabla\left(\frac{u_{i}}{u_{j}}\right), \nabla u_{j}\right) u_{j}^{-1} .
$$

Applying the maximum principle we get (3-3).

The main objective now is to prove that

$$
\min _{\partial B_{0}(R)} \frac{u_{i}}{u_{j}} \rightarrow \lambda_{i, j} \quad \text { and } \quad \max _{\partial B_{0}(R)} \frac{u_{i}}{u_{j}} \rightarrow \lambda_{i, j}
$$

as $R \rightarrow+\infty$ for some $\lambda_{i, j}>0$ so that, together with Step 1 , we obtain $u_{i}=\lambda_{i, j} u_{j}$ in $\mathbb{R}^{n}$ for all $i, j=1, \ldots p^{\prime}$. To prove (3-4) we first observe that

$$
|x|^{(n-2) / 2} u_{i}(x) \rightarrow 0
$$


as $|x| \rightarrow+\infty$ for all $i \in\left\{1, \ldots, p^{\prime}\right\}$. Indeed, let $r>0$, and $V_{r}=r^{(n-2) / 2} U(r x)$. We have $\Delta V_{r}=\left|V_{r}\right|^{2^{\star}-2} V_{r}$ and

$$
\int_{B_{0}(2) \backslash B_{0}(1 / 2)}\left|V_{r}\right|^{2^{\star}} d x \rightarrow 0 \quad \text { as } r \rightarrow+\infty,
$$

since $u_{i} \in L^{2^{\star}}(\mathbb{R})$ for all $i$. Then $v_{r}^{i} \rightarrow 0$ in $C_{\text {loc }}^{0}\left(B_{0}\left(\frac{3}{2}\right) \backslash B_{0}\left(\frac{3}{4}\right)\right)$ as $r \rightarrow+\infty$ for all $i$, where the $v_{r}^{i}$, s are the components of $V_{r}$. This proves (3-5). Now, in order to prove (3-4), we prove that the following step holds true.

Step 2. Let $U \in \dot{H}^{1}\left(\mathbb{R}^{n}\right)$ be a nonnegative solution of (3-1) such that $u_{i}>0$ in $\mathbb{R}^{n}$ for all $i=1, \ldots, p^{\prime}$, $p^{\prime} \leq p$. For any $0<\varepsilon<\frac{1}{2}$, there exists $C_{\varepsilon}>0$ such that

$$
u_{i}(x) \leq C_{\varepsilon}|x|^{(2-n)(1-\varepsilon)}
$$

for all $x \in \mathbb{R}^{n}$ and all $i \in\left\{1, \ldots, p^{\prime}\right\}$.

Proof of Step 2. Let $0<\varepsilon<\frac{1}{2}$ and $R_{\varepsilon}>0$ be such that

$$
\sup _{x \in \mathbb{R}^{n} \backslash B_{0}\left(R_{\varepsilon}\right)}|x|^{2}|U(x)|^{2^{\star}-2} \leq \frac{(n-2)^{2}}{2} \varepsilon(1-\varepsilon) .
$$

It is always possible to find such a $R_{\varepsilon}$ thanks to (3-5). For $R \geq R_{\varepsilon}$, we let

$$
\eta(R)=\max _{i=1, \ldots, p^{\prime}} \max _{\partial B_{0}(R)} u_{i}
$$

and

$$
G_{\varepsilon}(x)=\eta\left(R_{\varepsilon}\right)\left(\frac{|x|}{R_{\varepsilon}}\right)^{(2-n)(1-\varepsilon)}+\eta(R)\left(\frac{|x|}{R}\right)^{(2-n) \varepsilon} .
$$

It is clear that $u_{i} \leq G_{\varepsilon}$ on $\partial B_{0}\left(R_{\varepsilon}\right) \bigcup \partial B_{0}(R)$. Let us assume that $\frac{u_{i}}{G_{\varepsilon}}$ possesses a local maximum at $x \in B_{0}(R) \backslash \overline{B_{0}\left(R_{\varepsilon}\right)}$. Then

Since

$$
\frac{\Delta u_{i}(x)}{u_{i}(x)} \geq \frac{\Delta G_{\varepsilon}(x)}{\left.G_{\varepsilon}(x)\right)} .
$$

$$
\frac{\Delta G_{\varepsilon}(x)}{G_{\varepsilon}(x)}=\varepsilon(1-\varepsilon)(n-2)^{2}|x|^{-2},
$$

we get

$$
|x|^{2}|U(x)|^{2^{\star}-2} \geq \varepsilon(1-\varepsilon)(n-2)^{2} .
$$

But this is absurd by the choice of $R_{\varepsilon}$ we made. Thus we can write, for any $R>R_{\varepsilon}$ and any $i \in\left\{1, \ldots, p^{\prime}\right\}$,

$$
u_{i}(x) \leq \eta\left(R_{\varepsilon}\right)\left(\frac{|x|}{R_{\varepsilon}}\right)^{(2-n)(1-\varepsilon)}+\eta(R)\left(\frac{|x|}{R}\right)^{(2-n) \varepsilon}
$$

in $B_{0}(R) \backslash B_{0}\left(R_{\varepsilon}\right)$. Fix $x \in \mathbb{R}^{n} \backslash B_{0}\left(R_{\varepsilon}\right)$. Passing to the limit as $R \rightarrow+\infty$ in (3-6), since, by (3-5), $R^{(n-2) / 2} \eta(R) \rightarrow 0$ as $R \rightarrow+\infty$, we get

$$
u_{i}(x) \leq \eta\left(R_{\varepsilon}\right)\left(\frac{|x|}{R_{\varepsilon}}\right)^{(2-n)(1-\varepsilon)} .
$$

This ends the proof of Step 2. 
Step 3. Let $u \in \dot{H}^{1}\left(\mathbb{R}^{n}\right)$ be a nonnegative solution of (3-1) such that $u_{i}>0$ in $\mathbb{R}^{n}$ for all $i=1, \ldots, p^{\prime}$, $p^{\prime} \leq p$. Then $u_{i} \in L^{2^{\star}-1}\left(\mathbb{R}^{n}\right)$ and

$$
\lim _{|x| \rightarrow+\infty}|x|^{n-2} u_{i}(x)=\frac{1}{(n-2) \omega_{n-1}} \int_{\mathbb{R}^{n}}|U|^{2^{\star}-2} u_{i} d x
$$

for all $i \in\left\{1, \ldots, p^{\prime}\right\}$.

Proof of Step 3. We apply Green's representation formula in $B_{x}(R)$ and get

$$
u_{i}(x)=\frac{1}{(n-2) \omega_{n-1}} \int_{B_{x}(R)}\left(|x-y|^{2-n}-R^{2-n}\right)|U(y)|^{2^{\star}-2} u_{i}(y) d y+\frac{1}{\omega_{n-1} R^{n-1}} \int_{\partial B_{x}(R)} u_{i} d \sigma .
$$

Thanks to the estimate of Step 2 with $0<\varepsilon<2 /(n+2)$, we have $u_{i} \in L^{2^{\star}-1}\left(\mathbb{R}^{n}\right)$ for all $i$. Passing to the limit as $R \rightarrow+\infty$ we obtain

$$
u_{i}(x)=\frac{1}{(n-2) \omega_{n-1}} \int_{\mathbb{R}^{n}}|x-y|^{2-n}|U(y)|^{2^{\star}-2} u_{i}(y) d y .
$$

Thus

$$
\begin{aligned}
|x|^{n-2} u_{i} & (x) \\
\quad & =\frac{1}{(n-2) \omega_{n-1}} \int_{\mathbb{R}^{n}} \frac{|x|^{n-2}}{|x-y|^{n-2}}|U(y)|^{2^{\star}-2} u_{i}(y) d y \\
& =\frac{1}{(n-2) \omega_{n-1}}\left(\int_{B_{0}(R)}|U(y)|^{2^{\star}-2} u_{i}(y) d y+o_{R}(1)+\int_{\mathbb{R}^{n} \backslash B_{0}(R)} \frac{|x|^{n-2}}{|x-y|^{n-2}}|U(y)|^{2^{\star}-2} u_{i}(y) d y\right),
\end{aligned}
$$

where $o_{R}(1) \rightarrow 0$ as $|x| \rightarrow+\infty$. Now, using Step 2, we write

$$
\begin{aligned}
& \int_{\mathbb{R}^{n} \backslash B_{0}(R)} \frac{|x|^{n-2}}{|x-y|^{n-2}}|U(y)|^{2^{\star}-2} u_{i}(y) d y \\
& \quad \leq N^{\left(2^{\star}-2\right) / 2} C_{\varepsilon}^{2^{\star}-1} \int_{B_{x}(|x| / 2)} \frac{|x|^{n-2}}{|x-y|^{n-2}} d y\left(\frac{|x|}{2}\right)^{-(n+2)(1-\varepsilon)}+2^{n-2} \int_{\mathbb{R}^{n} \backslash B_{0}(R)}|U(y)|^{2^{\star}-2} u_{i}(y) d y \\
& \quad \leq N^{\left(2^{\star}-2\right) / 2} C_{\varepsilon}^{2^{\star}-1} 2^{(n+2)(1-\varepsilon)-2} \omega_{n-1}|x|^{n-(n+2)(1-\varepsilon)}+2^{n-2} \int_{\mathbb{R}^{n} \backslash B_{0}(R)}|U(y)|^{2^{\star}-2} u_{i}(y) d y .
\end{aligned}
$$

Choosing $0<\varepsilon<\frac{2}{n+2}$, we thus obtain that

$$
\lim _{R \rightarrow+\infty} \limsup _{|x| \rightarrow+\infty} \int_{\mathbb{R}^{n} \backslash B_{0}(R)} \frac{|x|^{n-2}}{|x-y|^{n-2}}|U(y)|^{2^{\star}-2} u_{i}(y) d y=0 .
$$

This ends the proof of Step 3.

Using Steps 1 and 3 we are now in a position to prove (3-4), and then Proposition 3.1.

Proof of Proposition 3.1. Let $U \in \dot{H}^{1}\left(\mathbb{R}^{n}\right)$ be a nonnegative solution of (3-1) such that $u_{i}>0$ in $\mathbb{R}^{n}$ for all $i=1, \ldots, p^{\prime}, p^{\prime} \leq p$. Since the $u_{i}$ 's are all positive for $i=1, \ldots, p^{\prime}$, we get from Step 3 that

$$
\min _{\partial B_{0}(R)} \frac{u_{i}}{u_{j}}, \max _{\partial B_{0}(R)} \frac{u_{i}}{u_{j}} \rightarrow \lambda_{i, j}
$$


as $R \rightarrow+\infty$, where $\lambda_{i, j}>0$ is given by

$$
\lambda_{i, j}=\frac{\int_{\mathbb{R}^{n}}|U|^{2^{\star}-2} u_{i} d x}{\int_{\mathbb{R}^{n}}|U|^{2^{\star}-2} u_{j} d x} .
$$

In particular, (3-4) holds true. Thanks to Step 1, we thus get

$$
u_{i}=\lambda_{i} u_{1}
$$

for all $i \in\left\{1, \ldots, p^{\prime}\right\}$ where $\lambda_{i}=\lambda_{i, 1}$. By (3-1) we then get

$$
\Delta u_{1}=\left|\Lambda^{\prime}\right|^{2^{\star}-2} u_{1}^{2^{\star}-1}
$$

in $\mathbb{R}^{n}$ where $\Lambda^{\prime}=\left(\lambda_{i}\right)_{i=1, \ldots, p^{\prime}}$. By [Caffarelli et al. 1989] we can write

$$
u_{1}(x)=\left|\Lambda^{\prime}\right|^{-1}\left(\frac{\mu}{\mu^{2}+\frac{\left|x-x_{0}\right|^{2}}{n(n-2)}}\right)^{(n-2) / 2}
$$

for some $x_{0} \in \mathbb{R}^{n}$ and some $\mu>0$. In particular, since $u_{i}=\lambda_{i} u_{1}$, we get with (3-7) that (3-2) holds true with $\Lambda=\left(\Lambda_{i}\right)_{i}$, where $\Lambda_{i}=\left|\Lambda^{\prime}\right|^{-1} \lambda_{i}$ for all $i=1, \ldots, p^{\prime}$, and $\lambda_{i}=0$ for all $i>p^{\prime}$. Clearly, $|\Lambda|=1$. This ends the proof of Proposition 3.1.

Let $K_{n}$ be the sharp constant for the Sobolev inequality $\|u\|_{2^{\star}} \leq K\|\nabla u\|_{2}$ corresponding to the embedding $\dot{H}^{1}\left(\mathbb{R}^{n}\right) \subset L^{2^{\star}}\left(\mathbb{R}^{n}\right)$. Then, as is well known,

$$
K_{n}=\sqrt{\frac{4}{n(n-2) \omega_{n}^{2 / n}}},
$$

where $\omega_{n}$ is the volume of the unit sphere. The multipliers in (3-2), which we get by taking the Euclidean norm $|u|$ of $u$ in (3-2), turn out to be extremal functions for the sharp Euclidean Sobolev inequality $\|u\|_{2^{\star}} \leq K_{n}\|\nabla u\|_{2}$. As a direct consequence of Proposition 3.1 we then get

$$
\int_{\mathbb{R}^{n}}|u|^{2^{\star}} d x=K_{n}^{-n}
$$

for all nonnegative solutions $u \in \dot{H}^{1}\left(\mathbb{R}^{n}\right)$ of (3-1), where $K_{n}$ is as in (3-8). Proposition 3.1, combined with the moving sphere approach, gives the full classification of nonnegative solutions of (3-1), namely without the requirement that $\boldsymbol{U} \in \dot{H}^{1}$. This is carried out in [Druet et al. 2009].

\section{Weak pointwise estimates}

Let $(M, g)$ be a smooth compact Riemannian manifold of dimension $n \geq 3, p \geq 1$ be an integer, and $\left(x_{\alpha}\right)_{\alpha}$ be a converging sequence of points in $M$. Let also $\left(\lambda_{\alpha}\right)_{\alpha}$ be a sequence of positive real numbers. For $\mathcal{U}: M \rightarrow \mathbb{R}^{p}$ and $\mathscr{V}: \mathbb{R}^{n} \rightarrow \mathbb{R}^{p}$, we define the direct $\hat{R}_{x_{\alpha}}^{\lambda_{\alpha}}$-rescalings and the inverse $\check{R}_{x_{\alpha}}^{\lambda_{\alpha}}$-rescalings by

$$
\left(\hat{R}_{x_{\alpha}}^{\lambda_{\alpha}} \mathcal{U}\right)(x)=\lambda_{\alpha}^{(n-2) / 2} \cup\left(\exp _{x_{\alpha}}\left(\lambda_{\alpha} x\right)\right) \quad \text { and } \quad\left(\check{R}_{x_{\alpha}}^{\lambda_{\alpha} \mathscr{V}}\right)(x)=\lambda_{\alpha}^{(n-2) / 2} \mathscr{V}\left(\lambda_{\alpha} \exp _{x_{\alpha}}^{-1}(x)\right),
$$

where $x$ in the first equation is a variable in $\mathbb{R}^{n}, x$ in the second equation is a variable in $M$, localized around the limit of the $x_{\alpha}$ 's, and $\exp _{x_{\alpha}}$ is the exponential map at $x_{\alpha}$. 
Definition 4.1. Let $(M, g)$ be a smooth compact Riemannian manifold of dimension $n \geq 3$ and $p \geq 1$ be an integer. A $p$-vector bubble is a sequence $\left(\mathscr{B}_{\alpha}\right)_{\alpha}$ of $p$-maps from $M$ to $\mathbb{R}^{p}$ given by

$$
\mathscr{B}_{\alpha}(x)=\left(\frac{\mu_{\alpha}}{\mu_{\alpha}^{2}+\frac{d_{g}\left(x_{\alpha}, x\right)^{2}}{n(n-2)}}\right)^{(n-2) / 2} \Lambda
$$

for all $x \in M$ and all $\alpha$, where $\left(x_{\alpha}\right)_{\alpha}$ is a converging sequence of points in $M,\left(\mu_{\alpha}\right)_{\alpha}$ is a sequence of positive real numbers converging to 0 , and $\Lambda \in S_{+}^{p-1}$. The $x_{\alpha}$ 's are the centers of the bubble, the $\mu_{\alpha}$ 's are the weights of the bubble, and $\Lambda$ is the $S^{p-1}$-projection of the bubble.

The right-hand side in (4-2) can be seen as the Riemannian extension of the right-hand side in (3-2). At last we let $u_{0}: \mathbb{R}^{n} \rightarrow \mathbb{R}$ be the function given by

$$
u_{0}(x)=\left(1+\frac{|x|^{2}}{n(n-2)}\right)^{-(n-2) / 2}
$$

for all $x \in \mathbb{R}^{n}$. Another possible definition of $u_{0}$ is that it is the unique nonnegative solution of $\Delta u=u^{2^{\star}-1}$ which achieves its maximum at 0 and which is such that $u_{0}(0)=1$. The result we prove in this section provides a complete description of the blow-up in Sobolev spaces and very useful pointwise estimates.

Proposition 4.2. Let $(M, g)$ be a smooth compact Riemannian manifold of dimension $n \geq 3, p \geq 1$ be an integer, and $\left(A_{\alpha}\right)_{\alpha}$ be a sequence of $C^{1}$ maps from $M$ to $M_{p}^{s}(\mathbb{R})$ such that $A_{\alpha} \rightarrow A$ in $C^{1}(M)$ as $\alpha \rightarrow+\infty$ for some $C^{1}$ map $A$ from $M$ to $M_{p}^{s}(\mathbb{R})$. Let also $\left(u_{\alpha}\right)_{\alpha}$ be an arbitrary bounded sequence in $H^{1}(M)$ of nonnegative solutions of (1-1) such that $\left\|u_{\alpha}\right\|_{\infty} \rightarrow+\infty$ as $\alpha \rightarrow+\infty$. Then there exist $N \in \mathbb{N}^{\star}$, a nonnegative solution $U_{\infty}$ of $(0-1)$, and vector bubbles $\left(\mathscr{B}_{\alpha}^{i}\right)_{\alpha}$ as in (4-2) for $i=1, \ldots, N$, such that, up to a subsequence,

$$
\begin{aligned}
& u_{\alpha}=u_{\infty}+\sum_{i=1}^{N} \mathscr{M}_{\alpha}^{i}+\mathscr{R}_{\alpha} \quad \text { for all } \alpha, \\
& \int_{M}\left|u_{\alpha}\right|^{2^{\star}} d v_{g}=\int_{M}\left|u_{\infty}\right|^{2^{\star}} d v_{g}+N K_{n}^{-n}+o(1) \quad \text { for all } \alpha, \text { and } \\
& \mathscr{D}_{\alpha}^{(n-2) / 2}\left|u_{\alpha}-u_{\infty}-\sum_{i=1}^{N} \mathscr{B}_{\alpha}^{i}\right| \rightarrow 0 \quad \text { in } L^{\infty}(M) \text { as } \alpha \rightarrow+\infty \text {, }
\end{aligned}
$$

where $\mathscr{R}_{\alpha} \rightarrow 0$ in $H^{1}(M)$ as $\alpha \rightarrow+\infty, o(1) \rightarrow 0$ as $\alpha \rightarrow+\infty, K_{n}$ is as in (3-8), $\mathscr{D}_{\alpha}: M \rightarrow \mathbb{R}^{+}$is given by

$$
\mathscr{D}_{\alpha}(x)=\min _{i=1, \ldots, N}\left(d_{g}\left(x_{i, \alpha}, x\right)+\mu_{i, \alpha}\right),
$$

and the $x_{i, \alpha}$ 's and $\mu_{i, \alpha}$ 's are the centers and weights of the vector bubbles $\left(\mathscr{S}_{\alpha}^{i}\right)_{\alpha}$. Moreover, as $\alpha \rightarrow+\infty$,

$$
\begin{array}{ll}
\frac{d_{g}\left(x_{i, \alpha}, x_{j, \alpha}\right)^{2}}{\mu_{i, \alpha} \mu_{j, \alpha}}+\frac{\mu_{i, \alpha}}{\mu_{j, \alpha}}+\frac{\mu_{j, \alpha}}{\mu_{i, \alpha}} \rightarrow+\infty & \text { for all } i \neq j \quad \text { and } \\
\hat{R}_{x_{i, \alpha}}^{\mu_{i, \alpha}} u_{\alpha} \rightarrow u_{0} \Lambda_{i} \text { in } C_{\mathrm{loc}}^{2}\left(\mathbb{R}^{n} \backslash \mathscr{S}_{i}\right) & \text { for all } i,
\end{array}
$$


where the $\hat{R}_{x_{\alpha}}^{\mu_{\alpha}}$-rescaling procedure is defined in (4-1), $u_{0}$ is as in (4-3), the $\Lambda_{i}$ 's are the $S^{p-1}$-projections of the $\left(\mathscr{B}_{\alpha}^{i}\right)_{\alpha}$ 's,

$$
\mathscr{S}_{i}=\left\{\lim _{\alpha \rightarrow+\infty} \mu_{i, \alpha}^{-1} \exp _{x_{i, \alpha}}^{-1}\left(x_{j, \alpha}\right), j \in I_{i}\right\},
$$

the limits in the definition of $\mathscr{Y}_{i}$ are as $\alpha \rightarrow+\infty$, and $I_{i}$ consists of the $j$ 's such that $d_{g}\left(x_{i, \alpha}, x_{j, \alpha}\right)=$ $O\left(\mu_{i, \alpha}\right)$ and $\mu_{j, \alpha}=o\left(\mu_{i, \alpha}\right)$ for all $\alpha$.

Proof. Let $I_{\alpha}$ be the free functionals associated with (0-1). They are defined for $U \in H^{1}(M)$ by

$$
I_{\alpha}(u)=\frac{1}{2} \int_{M}\left(|\nabla \cup|^{2}+A_{\alpha}(u, \mathcal{u})\right) d v_{g}-\frac{1}{2^{\star}} \int_{M}|u|^{2^{\star}} d v_{g} .
$$

The $u_{\alpha}$ 's in Proposition 4.2 solve $(0-1)$ and are bounded in $H^{1}$. In particular, $\left(u_{\alpha}\right)_{\alpha}$ is a Palais-Smale sequence for the $I_{\alpha}$ 's in the sense that the sequence $\left(I_{\alpha}\left(U_{\alpha}\right)\right)_{\alpha}$ is bounded and $D I_{\alpha}\left(U_{\alpha}\right) \rightarrow 0$ in $H^{1}(M)^{\prime}$ as $\alpha \rightarrow+\infty$. Let $\eta$ be a smooth cutoff function in $\mathbb{R}^{n}$ with small support around 0 . Mimicking the proof in [Struwe 1984] (see also [Druet et al. 2004] for its Riemannian analogue), we get that there exist $N \in \mathbb{N}^{\star}$, a nonnegative solution $U_{\infty}$ of (0-1), converging sequences $\left(x_{i, \alpha}\right)_{\alpha}$ in $M$, sequences $\left(\mu_{i, \alpha}\right)_{\alpha}$ of positive real numbers converging to 0 , and nonnegative solutions $u_{i} \in \dot{H}^{1}\left(\mathbb{R}^{n}\right)$ of (3-1) in $\mathbb{R}^{n}, i=1, \ldots, N$, such that, up to a subsequence, the first equation in (4-5) holds true, such that

$$
u_{\alpha}=u_{\infty}+\sum_{i=1}^{N} \eta_{\alpha}^{i} \check{R}_{x_{i, \alpha}}^{1 / \mu_{i, \alpha}} u_{i}+\mathscr{R}_{\alpha}
$$

for all $\alpha$, and such that

$$
\int_{M}\left|u_{\alpha}\right|^{2^{\star}} d v_{g}=\int_{M}\left|u_{\infty}\right|^{2^{\star}} d v_{g}+\sum_{i=1}^{N} \int_{\mathbb{R}^{n}}\left|u_{i}\right|^{2^{\star}} d x+o(1)
$$

for all $\alpha$, where $\eta_{\alpha}^{i}(x)=\eta\left(\exp _{x_{i, \alpha}}^{-1}(x)\right)$, the $\check{R}_{x_{i, \alpha}}^{1 / \mu_{i, \alpha}}$-rescalings are defined in (4-1), $\mathscr{R}_{\alpha} \rightarrow 0$ in $H^{1}(M)$ as $\alpha \rightarrow+\infty$, and $o(1) \rightarrow 0$ as $\alpha \rightarrow+\infty$. By Proposition 3.1,

$$
u_{i}(x)=\left(\frac{\lambda_{i}}{\lambda_{i}^{2}+\frac{\left|x-a_{i}\right|}{n(n-2)}}\right)^{(n-2) / 2} \Lambda_{i}
$$

for some $a_{i} \in \mathbb{R}^{n}, \lambda_{i}>0, \Lambda_{i} \in S_{+}^{p-1}$, and all $x \in \mathbb{R}^{n}$. Up to changing the $x_{i, \alpha}$ 's and $\mu_{i, \alpha}$ 's, letting $\tilde{x}_{i, \alpha}=\exp _{x_{i, \alpha}}\left(\mu_{i, \alpha} a_{i}\right)$ and $\tilde{\mu}_{i, \alpha}=\lambda_{i} \mu_{i, \alpha}$, we can write, as in [Druet and Hebey 2005b], that

$$
\eta_{\alpha}^{i} \check{R}_{x_{i, \alpha}}^{1 / \mu_{i, \alpha}} u_{i}=\mathscr{S}_{\alpha}^{i}+\mathscr{R}_{\alpha}
$$

for all $\alpha$, where $U_{i}$ is as in (4-8), $\mathscr{R}_{\alpha} \rightarrow 0$ in $H^{1}(M)$ as $\alpha \rightarrow+\infty$, and $\left(\mathscr{B}_{\alpha}^{i}\right)_{\alpha}$ is the vector bubble with center $\tilde{x}_{i, \alpha}$, weight $\tilde{\mu}_{i, \alpha}$, and $S^{p-1}$-projection $\Lambda_{i}$. Noting that the changes $x_{i, \alpha} \rightarrow \tilde{x}_{i, \alpha}$ and $\mu_{i, \alpha} \rightarrow \tilde{\mu}_{i, \alpha}$ do not affect the first equation in (4-5), it follows from the above discussion, from (3-9), and from (4-6), (4-7), and (4-9), that the two first equations in (4-4) and the first equation in (4-5) hold true. Now we forget about the tilde notation for the centers and weights of the bubbles and, for $i=1, \ldots, N$, we let 
$\mathscr{Y}_{i}$ be as in Proposition 4.2. As one can check from the first equations in (4-4) and (4-5), for any $i$,

$$
\hat{R}_{x_{i, \alpha}}^{\mu_{i, \alpha}} \sum_{j=1}^{N} \mathscr{B}_{\alpha}^{j} \rightarrow u_{0} \Lambda_{i} \text { in } L_{\mathrm{loc}}^{\infty}\left(\mathbb{R}^{n} \backslash \mathscr{S}_{i}\right) \quad \text { and } \quad \hat{R}_{x_{i, \alpha}}^{\mu_{i, \alpha}} u_{\alpha}-\hat{R}_{x_{i, \alpha}}^{\mu_{i, \alpha}} \sum_{j=1}^{N} \mathscr{B}_{\alpha}^{j} \rightarrow 0 \text { in } L_{\mathrm{loc}}^{2^{\star}}\left(\mathbb{R}^{n}\right)
$$

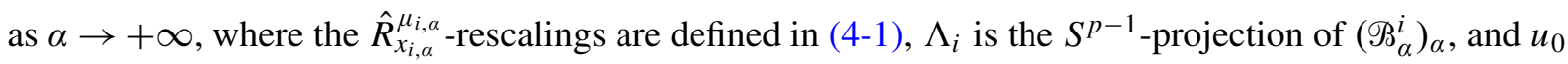
is as in (4-3). Moreover, in any compact subset of $\mathbb{R}^{n}$, and for $\alpha$ sufficiently large,

$$
\Delta_{g_{\alpha}} \tilde{u}_{i, \alpha}+\mu_{i, \alpha}^{2} \sum_{j=1}^{p} \tilde{A}_{i j}^{\alpha}(x) \tilde{u}_{j, \alpha}=\left|\tilde{u}_{\alpha}\right|^{2^{\star}-2} \tilde{u}_{i, \alpha}
$$

for all $\alpha$ and all $i$, where the $\tilde{u}_{i, \alpha}$ 's are the components of $\tilde{u}_{\alpha}=\hat{R}_{x_{i, \alpha}}^{\mu_{i, \alpha}} u_{\alpha}$,

$$
\tilde{A}_{i j}^{\alpha}(x)=A_{i j}^{\alpha}\left(\exp _{x_{i, \alpha}}\left(\mu_{i, \alpha} x\right)\right),
$$

and $g_{\alpha}$ is the Riemannian metric in $\mathbb{R}^{n}$ given by $g_{\alpha}(x)=\left(\exp _{x_{i, \alpha}}^{\star} g\right)\left(\mu_{i, \alpha} x\right)$. Since $\mu_{i, \alpha} \rightarrow 0$ as $\alpha \rightarrow+\infty$, we get that $g_{\alpha} \rightarrow \xi$ in $C_{\mathrm{loc}}^{2}\left(\mathbb{R}^{n}\right)$ as $\alpha \rightarrow+\infty$, where $\xi$ is the Euclidean metric. By (4-10), for any $x \in \mathbb{R}^{n} \backslash \mathscr{Y}_{i}$,

$$
\lim _{\delta \rightarrow 0} \limsup _{\alpha \rightarrow+\infty} \int_{B_{x}(\delta)}\left|\hat{R}_{x_{i, \alpha}}^{\mu_{i, \alpha}} u_{\alpha}\right|^{2^{\star}} d x=0 .
$$

In particular, the $L^{2^{\star}}$-norm of $\hat{R}_{x_{i, \alpha}}^{\mu_{i, \alpha}} U_{\alpha}$ can be made uniformly arbitrarily small in small regions of $\mathbb{R}^{n} \backslash \mathscr{S}_{i}$, and by adapting and transposing the classical regularity argument [Trudinger 1968] to the present situation (see also [Struwe 1990]) we get from (4-11) and (4-12) that the $\tilde{U}_{\alpha}$ 's are uniformly bounded in $C_{\text {loc }}^{2, \theta}\left(\mathbb{R}^{n} \backslash \mathscr{S}_{i}\right)$. It easily follows that, up to a subsequence, the second equation in (4-5) also holds true. Now it remains to prove that the third equation in (4-4) holds true. We proceed by contradiction and assume that there exists $\varepsilon_{0}>0$ and a sequence $\left(x_{\alpha}\right)_{\alpha}$ in $M$ such that, up to a subsequence,

$$
\mathscr{D}_{\alpha}\left(x_{\alpha}\right)^{2}\left|u_{\alpha}\left(x_{\alpha}\right)-u_{\infty}\left(x_{\alpha}\right)-\sum_{i=1}^{N} \mathscr{P}_{\alpha}^{i}\left(x_{\alpha}\right)\right|^{2^{\star}-2}=\max _{M} \mathscr{D}_{\alpha}^{2}\left|u_{\alpha}-u_{\infty}-\sum_{i=1}^{N} \mathscr{P}_{\alpha}^{i}\right|^{2^{\star}-2} \geq 4 \varepsilon_{0}
$$

for all $\alpha$. First we claim that

$$
\mathscr{D}_{\alpha}\left(x_{\alpha}\right)^{2}\left|\mathscr{R}_{\alpha}^{i}\left(x_{\alpha}\right)\right|^{2^{\star}-2} \rightarrow 0
$$

as $\alpha \rightarrow+\infty$, for all $i=1, \ldots, N$. In order to prove (4-14) we proceed by contradiction and assume that there exists $i=1, \ldots, N$ and $\varepsilon_{1}>0$ such that, up to a subsequence,

$$
\mathscr{D}_{\alpha}\left(x_{\alpha}\right)^{2}\left|\Re_{\alpha}^{i}\left(x_{\alpha}\right)\right|^{2^{\star}-2} \geq \varepsilon_{1}
$$

for all $\alpha$. Up to passing to another subsequence we may then assume that there is $\lambda \in[0,+\infty)$ such that

$$
\frac{d_{g}\left(x_{i, \alpha}, x_{\alpha}\right)}{\mu_{i, \alpha}} \rightarrow \lambda \text { as } \alpha \rightarrow+\infty, \quad \text { and } \quad \frac{\mu_{j, \alpha}}{\mu_{i, \alpha}}+\frac{d_{g}\left(x_{j, \alpha}, x_{\alpha}\right)}{\mu_{i, \alpha}} \geq \sqrt{\varepsilon_{1}} \text { for all } \alpha \text { and } j .
$$

Then, letting $y_{\alpha}=\mu_{i, \alpha}^{-1} \exp _{x_{i, \alpha}}^{-1}\left(x_{\alpha}\right)$, we get from the second equation in (4-16) that there exists $\varepsilon>0$ such that $d\left(y_{\alpha}, \mathscr{S}_{i}\right) \geq \varepsilon$ for all $\alpha$, and it follows from the second equation in (4-5) that

$$
\mathscr{D}_{\alpha}\left(x_{\alpha}\right)^{2}\left|u_{\alpha}\left(x_{\alpha}\right)-u_{\infty}\left(x_{\alpha}\right)-\mathscr{B}_{\alpha}^{i}\left(x_{\alpha}\right)\right|^{2^{\star}-2} \rightarrow 0 \quad \text { as } \alpha \rightarrow+\infty \text {. }
$$


By the first equation in (4-5), and by (4-16), we can also write

$$
\mathscr{D}_{\alpha}\left(x_{\alpha}\right)^{2}\left|\mathscr{B}_{\alpha}^{j}\left(x_{\alpha}\right)\right|^{2^{\star}-2} \rightarrow 0
$$

as $\alpha \rightarrow+\infty$, for all $j \neq i$. Combining (4-17) and (4-18) we get a contradiction with (4-13). It follows that (4-14) holds true. Next we claim that

$$
\left|u_{\alpha}\left(x_{\alpha}\right)\right| \rightarrow+\infty \quad \text { as } \alpha \rightarrow+\infty \text {. }
$$

By (4-13) and (4-14), we see that (4-19) holds if $\mathscr{D}_{\alpha}\left(x_{\alpha}\right) \rightarrow 0$ as $\alpha \rightarrow+\infty$. Suppose on the contrary that, up to a subsequence, $\mathscr{D}_{\alpha}\left(x_{\alpha}\right) \rightarrow \delta$ as $\alpha \rightarrow+\infty$ for some $\delta>0$. Then, by (4-13) and (4-14),

$$
\left|u_{\alpha}(x)-u_{\infty}(x)\right|^{2^{\star}-2}+o(1) \leq 8\left|u_{\alpha}\left(x_{\alpha}\right)-u_{\infty}\left(x_{\alpha}\right)\right|^{2^{\star}-2}+o(1)
$$

for all $x \in B_{x_{\alpha}}(\delta / 2)$ and all $\alpha$ sufficiently large. Now, if we assume that (4-19) is false, then we get from (4-20) that the $u_{\alpha}$ 's are bounded in a neighbourhood of the $x_{\alpha}$ 's, and it follows from standard elliptic theory that $u_{\alpha}\left(x_{\alpha}\right)-u_{\infty}\left(x_{\alpha}\right) \rightarrow 0$ as $\alpha \rightarrow+\infty$. Noting that this convergence of the $\left(u_{\alpha}-u_{\infty}\right)\left(x_{\alpha}\right)$ 's is in contradiction with (4-13) and (4-14), we obtain (4-19).

Now let the $\mu_{\alpha}$ 's be given by $\mu_{\alpha}^{1-(n / 2)}=\left|\mathcal{U}_{\alpha}\left(x_{\alpha}\right)\right|$ for all $\alpha$, and define the $\mathscr{V}_{\alpha}$ 's by $\mathscr{V}_{\alpha}=\hat{R}_{x_{\alpha}}^{\mu_{\alpha}}{U_{\alpha}}_{\alpha}$, where the $\hat{R}_{x_{\alpha}}^{\mu_{\alpha}}$-rescalings are defined in (4-1). Then,

$$
\Delta_{g_{\alpha}} v_{i, \alpha}+\mu_{\alpha}^{2} \sum_{j=1}^{p} \hat{A}_{i j}^{\alpha}(x) v_{j, \alpha}=\left|\mathscr{V}_{\alpha}\right|^{2^{\star}-2} v_{i, \alpha}
$$

in $B_{0}\left(\delta / \mu_{\alpha}\right)$ for all $\alpha$, where the $v_{i, \alpha}$ 's are the components of $\mathscr{V}_{\alpha}$, the $\hat{A}_{i j}^{\alpha}$ 's are given by $\hat{A}_{i j}^{\alpha}(x)=$ $A_{i j}^{\alpha}\left(\exp _{x_{\alpha}}\left(\mu_{\alpha} x\right)\right)$, and $g_{\alpha}$ is given by $g_{\alpha}(x)=\left(\exp _{x_{\alpha}}^{\star} g\right)\left(\mu_{\alpha} x\right)$. From (4-19) we have $\mu_{\alpha} \rightarrow 0$ as $\alpha \rightarrow+\infty$. In particular, $g_{\alpha} \rightarrow \xi$ in $C_{\text {loc }}^{2}\left(\mathbb{R}^{n}\right)$ as $\alpha \rightarrow+\infty$. We also have $\left|\mathscr{V}_{\alpha}(0)\right|=1$ for all $\alpha$. Noting that the $\mathscr{V}_{\alpha}$ 's are bounded in $\dot{H}^{1}\left(\mathbb{R}^{n}\right)$, we may assume that, up to a subsequence, $\mathscr{V}_{\alpha} \rightarrow \mathscr{V}_{\infty}$ weakly in $H_{\text {loc }}^{1}\left(\mathbb{R}^{n}\right)$ as $\alpha \rightarrow+\infty$ for some $\mathscr{V}_{\infty} \in H^{1}\left(\mathbb{R}^{n}\right)$ that solves (3-1). Let $\tilde{\mathscr{S}}$ be given by

$$
\tilde{\mathscr{S}}=\left\{\lim _{\alpha \rightarrow+\infty} \frac{1}{\mu_{\alpha}} \exp _{x_{\alpha}}^{-1}\left(x_{i, \alpha}\right): i \in J\right\}
$$

where $J$ consists of the $i=1, \ldots, N$ which are such that $d_{g}\left(x_{i, \alpha}, x_{\alpha}\right)=O\left(\mu_{\alpha}\right)$ and $\mu_{i, \alpha}=o\left(\mu_{\alpha}\right)$ for all $\alpha$. In what follows we let $K \Subset \mathbb{R}^{n} \backslash \tilde{\mathscr{S}}$ be a compact subset of $\mathbb{R}^{n} \backslash \tilde{\mathscr{Y}}$, and let $x \in K$. By (4-13) and (4-14) we have

$$
\left|\mathscr{V}_{\alpha}(x)-\mu_{\alpha}^{(n-2) / 2} u_{\infty}\left(y_{\alpha}\right)-\mu_{\alpha}^{(n-2) / 2} \sum_{i=1}^{N} \Lambda_{i} B_{i, \alpha}\left(y_{\alpha}\right)\right|^{2^{\star}-2} \leq\left(\frac{\mathscr{D}_{\alpha}\left(x_{\alpha}\right)}{\mathscr{D}_{\alpha}\left(y_{\alpha}\right)}\right)^{2}(1+o(1))+o(1),
$$

where $y_{\alpha}=\exp _{x_{\alpha}}\left(\mu_{\alpha} x\right)$ for all $\alpha, \Lambda_{i}$ is the $S^{p-1}$-projection of $\left(\mathscr{B}_{\alpha}^{i}\right)_{\alpha}$ for all $i$, and $B_{i, \alpha}=\left|\mathscr{B}_{\alpha}^{i}\right|$ for all $\alpha$ and $i$. Now we claim that

$$
\mu_{\alpha}^{(n-2) / 2}\left|B_{i, \alpha}\left(y_{\alpha}\right)\right| \rightarrow 0
$$

as $\alpha \rightarrow+\infty$, for all $i=1, \ldots, N$. Equation (4-23) is obvious if $\mu_{\alpha}=o\left(\mu_{i, \alpha}\right)$. On the other hand, if we assume that $\mu_{i, \alpha}=o\left(\mu_{\alpha}\right)$, then, since $d_{\xi}(x, \tilde{\mathscr{S}})>0$, we get $\mu_{\alpha}=O\left(d_{g}\left(x_{i, \alpha}, y_{\alpha}\right)\right)$. Here again, (4-23) holds true. At last we may assume that there exists $C>0$ such that $C^{-1} \mu_{\alpha} \leq \mu_{i, \alpha} \leq C \mu_{\alpha}$ for all $\alpha$. 
Then (4-23) holds true unless $d_{g}\left(x_{i, \alpha}, y_{\alpha}\right)=O\left(\mu_{i, \alpha}\right)$. In this case we have $d_{g}\left(x_{i, \alpha}, x_{\alpha}\right)=O\left(\mu_{i, \alpha}\right)$, and it follows that $\left|B_{i, \alpha}\left(x_{\alpha}\right)\right| /\left|u_{\alpha}\left(x_{\alpha}\right)\right| \nrightarrow \supset 0$ as $\alpha \rightarrow+\infty$. Combining (4-13) and (4-14) we get a contradiction, and it follows that (4-23) holds true. In particular, by (4-19), (4-22), and (4-23), we can write

$$
\left|\mathscr{V}_{\alpha}(x)\right|^{2^{\star}-2} \leq\left(\frac{\mathscr{D}_{\alpha}\left(x_{\alpha}\right)}{\mathscr{D}_{\alpha}\left(y_{\alpha}\right)}\right)^{2}(1+o(1))+o(1) \text {. }
$$

At this point we claim that

$$
\mathscr{D}_{\alpha}\left(x_{\alpha}\right)=O\left(\mathscr{D}_{\alpha}\left(y_{\alpha}\right)\right) \text {. }
$$

We prove (4-25) by contradiction and assume that

$$
d_{g}\left(x_{i, \alpha}, y_{\alpha}\right)+\mu_{i, \alpha}=o\left(\mathscr{D}_{\alpha}\left(x_{\alpha}\right)\right) \text {. }
$$

If $d_{g}\left(x_{i, \alpha}, x_{\alpha}\right) / \mu_{\alpha} \rightarrow+\infty$ as $\alpha \rightarrow+\infty$, then

$$
d_{g}\left(x_{i, \alpha}, y_{\alpha}\right)+\mu_{i, \alpha} \geq(1+o(1)) d_{g}\left(x_{i, \alpha}, x_{\alpha}\right)+\mu_{i, \alpha} \geq(1+o(1)) \mathscr{D}_{\alpha}\left(x_{\alpha}\right),
$$

and this contradicts (4-26). Hence, $d_{g}\left(x_{i, \alpha}, x_{\alpha}\right)=O\left(\mu_{\alpha}\right)$. Then, by (4-26),

$$
d_{g}\left(x_{i, \alpha}, y_{\alpha}\right)+\mu_{i, \alpha}=o\left(\mu_{\alpha}\right)+o\left(\mu_{i, \alpha}\right) .
$$

In particular, $d_{g}\left(x_{i, \alpha}, y_{\alpha}\right)=o\left(\mu_{\alpha}\right)$. Since $x \in K$, this implies in turn that $\mu_{\alpha}=O\left(\mu_{i, \alpha}\right)$, and we get with (4-27) that $\mu_{i, \alpha}+o\left(\mu_{i, \alpha}\right)=0$, another contradiction. This proves (4-25). By (4-24) and (4-25), for any compact subset $K \Subset \mathbb{R}^{n} \backslash \tilde{\mathscr{S}}$, there exists $C_{K}>0$ such that $\left|\mathscr{V}_{\alpha}\right| \leq C_{K}$ in $K$. In particular, by standard elliptic theory and (4-21), we get

$$
\mathscr{V}_{\alpha} \rightarrow \mathscr{V}_{\infty} \text { in } C_{\mathrm{loc}}^{2}\left(\mathbb{R}^{n} \backslash \tilde{\mathscr{S}}\right) \quad \text { as } \alpha \rightarrow+\infty
$$

Clearly $0 \notin \tilde{\mathscr{S}}$ since, if not the case, $\mathscr{D}_{\alpha}\left(x_{\alpha}\right)=o\left(\mu_{\alpha}\right)$ and we get a contradiction with (4-13). Thus, since $\left|\mathscr{V}_{\alpha}(0)\right|=1$ for all $\alpha$, we see that $\left|\mathscr{V}_{\infty}(0)\right|=1$ and $\mathscr{V}_{\infty} \not \equiv 0$ is not identically zero. By Proposition 3.1 it follows that there exists $a \in \mathbb{R}^{n}, \lambda>0$, and $\Lambda \in S_{+}^{p-1}$, such that

$$
\mathscr{V}_{\infty}(x)=\left(\frac{\lambda}{\lambda^{2}+\frac{|x-a|}{n(n-2)}}\right)^{(n-2) / 2} \Lambda
$$

for all $x \in \mathbb{R}^{n}$. Let $K \Subset \mathbb{R}^{n} \backslash \tilde{\mathscr{S}}$ be a nonempty compact subset of $\mathbb{R}^{n} \backslash \tilde{\mathscr{T}}$. By the first equation in (4-4) and by (4-23), we can write $\mathscr{V}_{\alpha} \rightarrow 0$ in $L^{2^{\star}}(K)$ as $\alpha \rightarrow+\infty$. Then, by (4-28), we get $\int_{K}\left|\mathscr{V}_{\infty}\right|^{2^{\star}} d x=0$, a contradiction with (4-29). Proposition 4.2 is proved.

\section{A first strong pointwise estimate}

We prove pointwise estimates on the $u_{\alpha}$ 's which we use as the initial step in the induction argument we develop in the next section. First we fix some notations. We let $\left(\mathcal{U}_{\alpha}\right)_{\alpha}$ be an arbitrary bounded sequence in $H^{1}(M)$ of nonnegative solutions of (1-1) such that $\left\|u_{\alpha}\right\|_{\infty} \rightarrow+\infty$ as $\alpha \rightarrow+\infty$. Proposition 4.2 applies to the $\boldsymbol{U}_{\alpha}$ 's. We let $\mathscr{S}$ be the set of the geometrical points of the $\boldsymbol{u}_{\alpha}$ 's. Then,

$$
\mathscr{S}=\left\{\lim _{\alpha \rightarrow+\infty} x_{i, \alpha}: i=1, \ldots, N\right\},
$$


where all the limits do exist, up to a subsequence. For $\delta>0$ small enough, we let

$$
\eta_{\alpha}(\delta)=\max _{M \backslash \bigcup_{i=1}^{N} B_{x_{i, \alpha}}(\delta)}\left|\mathcal{U}_{\alpha}\right| .
$$

Thanks to the last equation in (4-4) of Proposition 4.2

$$
\limsup _{\alpha \rightarrow+\infty} \eta_{\alpha}(\delta) \leq\left\|u_{\infty}\right\|_{\infty} .
$$

Moreover, by standard elliptic theory, for any $\delta^{\prime}>\delta$,

$$
\max _{M \backslash \bigcup_{i=1}^{N} B_{x_{i, \alpha}}\left(\delta^{\prime}\right)}\left|\nabla \mathcal{U}_{\alpha}\right|_{g}=O\left(\eta_{\alpha}(\delta)\right) .
$$

In what follows we let $R_{0}>0$ be such that for any $i=1, \ldots, N$,

$$
|x| \leq \frac{R_{0}}{2}
$$

for all $x \in \mathscr{Y}_{i}$, where $\mathscr{S}_{i}$ is as in Proposition 4.2. We also set

$$
\mu_{\alpha}=\max _{i \in\{1, \ldots, N\}} \mu_{i, \alpha}, \quad \text { and } \quad r_{\alpha}(x)=\min _{i \in\{1, \ldots, N\}} d_{g}\left(x_{i, \alpha}, x\right) .
$$

The pointwise estimate we prove in this section is stated as follows.

Proposition 5.1. Let $(M, g)$ be a smooth compact Riemannian manifold of dimension $n \geq 3, p \geq 1$ be an integer, and $\left(A_{\alpha}\right)_{\alpha}$ be a sequence of $C^{1}$ maps from $M$ to $M_{p}^{s}(\mathbb{R})$ such that $A_{\alpha} \rightarrow A$ in $C^{1}(M)$ as $\alpha \rightarrow+\infty$ for some $C^{1}$ map A from $M$ to $M_{p}^{s}(\mathbb{R})$ satisfying $(\mathrm{H})$. Let also $\left(\cup_{\alpha}\right)_{\alpha}$ be an arbitrary bounded sequence in $H^{1}(M)$ of nonnegative solutions of (1-1) such that $\left\|u_{\alpha}\right\|_{\infty} \rightarrow+\infty$ as $\alpha \rightarrow+\infty$. There exists $C_{1}>0$ such that, up to passing to a subsequence on the $u_{\alpha}$ 's, there holds that for any sequence $\left(x_{\alpha}\right)_{\alpha}$ of points in $M$,

$$
\left|u_{\alpha}\left(x_{\alpha}\right)-u_{\infty}\left(x_{\alpha}\right)\right| \leq C_{1} \mu_{\alpha}^{(n-2) / 2} \mathscr{D}_{\alpha}\left(x_{\alpha}\right)^{2-n}+\varepsilon_{\alpha}\left\|u_{\infty}\right\|_{\infty},
$$

where $\mathscr{D}_{\alpha}$ and $\boldsymbol{U}_{\infty}$ are as in Proposition 4.2, $\mu_{\alpha}$ is as in (5-6), and $\varepsilon_{\alpha} \rightarrow 0$ as $\alpha \rightarrow+\infty$.

We divide the proof of Proposition 5.1 into two steps.

Step 1. For any $0<\varepsilon<\frac{1}{2}$, there exist $R_{\varepsilon}>0, \delta_{\varepsilon}>0$ and $C_{\varepsilon}>0$ such that

$$
\left|u_{\alpha}(x)\right| \leq C_{\varepsilon}\left(\mu_{\alpha}^{(1-2 \varepsilon)(n-2) / 2} r_{\alpha}(x)^{(2-n)(1-\varepsilon)}+\eta_{\alpha}\left(\delta_{\varepsilon}\right) r_{\alpha}(x)^{(2-n) \varepsilon}\right)
$$

for all $\alpha$ and all $x \in M \backslash \bigcup_{i=1}^{N} B_{x_{i, \alpha}}\left(R_{\varepsilon} \mu_{i, \alpha}\right)$.

Proof of Step 1. Let $0<\varepsilon<\frac{1}{2}$. Consider $\mathscr{G}$ the Green's function of the operator $u \mapsto \Delta_{g} u+u$. We know (see [Druet et al. 2004], for example) that there exist $\gamma_{1}>1, \gamma_{2}>0$ and $\gamma_{3}>0$ such that for any distinct $x, y \in M$,

$$
\frac{1}{\gamma_{1}} \leq d_{g}(x, y)^{n-2} \varphi(x, y) \leq \gamma_{1}
$$

and

$$
\frac{|\nabla \mathscr{G}(x, y)|^{2}}{\mathscr{G}(x, y)^{2}} \geq \gamma_{2} d_{g}(x, y)^{-2}-\gamma_{3},
$$


where $\nabla$ in (5-9) is with respect to one of the two variables, for instance $y$. We let

$$
\Psi_{\alpha, \varepsilon}(x)=\mu_{\alpha}^{(1-2 \varepsilon)(n-2) / 2} \sum_{i=1}^{N} \mathscr{G}\left(x_{i, \alpha}, x\right)^{1-\varepsilon}+\eta_{\alpha}(\delta) \sum_{i=1}^{N} \mathscr{G}\left(x_{i, \alpha}, x\right)^{\varepsilon},
$$

and let $y_{\alpha} \in M \backslash \bigcup_{i=1}^{N} B_{x_{i, \alpha}}\left(R \mu_{i, \alpha}\right)$ be such that

$$
\max _{M \backslash \bigcup_{i=1}^{p} B_{x_{i, \alpha}}\left(R \mu_{i, \alpha}\right)} \frac{\sum_{i=1}^{p} u_{i, \alpha}}{\Psi_{\alpha, \varepsilon}}=\frac{\sum_{i=1}^{N} u_{i, \alpha}}{\Psi_{\alpha, \varepsilon}}\left(y_{\alpha}\right)
$$

for all $\alpha$. We claim that, if $\delta>0$ is chosen sufficiently small and $R>0$ sufficiently large, then

$$
y_{\alpha} \in \partial\left(M \backslash \bigcup_{i=1}^{N} B_{x_{i, \alpha}}\left(R \mu_{i, \alpha}\right)\right) \quad \text { or } \quad r_{\alpha}\left(y_{\alpha}\right)>\delta
$$

for $\alpha$ large. We prove the claim by contradiction. Indeed, assume that (5-11) fails for all $\alpha$. We can write

$$
\frac{\Delta_{g}\left(\sum_{i=1}^{p} u_{i, \alpha}\right)}{\sum_{i=1}^{p} u_{i, \alpha}}\left(y_{\alpha}\right) \geq \frac{\Delta_{g} \Psi_{\alpha, \varepsilon}}{\Psi_{\alpha, \varepsilon}}\left(y_{\alpha}\right) .
$$

Thanks to (1-1),

$$
\frac{\Delta_{g}\left(\sum_{i=1}^{p} u_{i, \alpha}\right)}{\sum_{i=1}^{p} u_{i, \alpha}}\left(y_{\alpha}\right) \leq\left|u_{\alpha}\left(y_{\alpha}\right)\right|^{2^{\star}-2}+p\left\|A_{\alpha}\right\|_{\infty},
$$

where $\left\|A_{\alpha}\right\|_{\infty}=\max _{i, j}\left\|A_{i j}^{\alpha}\right\|_{\infty}$ for all $\alpha$. By (5-12) we then get $\frac{\Delta_{g} \Psi_{\alpha, \varepsilon}}{\Psi_{\alpha, \varepsilon}}\left(y_{\alpha}\right) \leq\left|u_{\alpha}\left(y_{\alpha}\right)\right|^{2^{\star}-2}+p\left\|A_{\alpha}\right\|_{\infty}$. Since $r_{\alpha}\left(y_{\alpha}\right) \leq \delta$, this yields

$$
r_{\alpha}\left(y_{\alpha}\right)^{2} \frac{\Delta_{g} \Psi_{\alpha, \varepsilon}}{\Psi_{\alpha, \varepsilon}}\left(y_{\alpha}\right) \leq r_{\alpha}\left(y_{\alpha}\right)^{2}\left|u_{\alpha}\left(y_{\alpha}\right)\right|^{2^{\star}-2}+\delta^{2} p\|A\|_{\infty}+o(1) .
$$

Now we write

$$
\begin{aligned}
\Delta_{g} \Psi_{\alpha, \varepsilon}\left(y_{\alpha}\right)=\varepsilon(1-\varepsilon) \mu_{\alpha}^{(1-2 \varepsilon)(n-2) / 2} & \sum_{i=1}^{N} \frac{\left|\nabla \mathscr{G}\left(x_{i, \alpha}, y_{\alpha}\right)\right|_{g}^{2}}{\mathscr{G}\left(x_{i, \alpha}, y_{\alpha}\right)^{2}} \mathscr{G}\left(x_{i, \alpha}, y_{\alpha}\right)^{1-\varepsilon} \\
+\varepsilon(1-\varepsilon) \eta_{\alpha}(\delta) & \sum_{i=1}^{N} \frac{\left|\nabla \mathscr{G}\left(x_{i, \alpha}, y_{\alpha}\right)\right|_{g}^{2}}{\mathscr{G}\left(x_{i, \alpha}, y_{\alpha}\right)^{2}} \mathscr{G}\left(x_{i, \alpha}, y_{\alpha}\right)^{\varepsilon} \\
& -\varepsilon \eta_{\alpha}(\delta) \sum_{i=1}^{N} \mathscr{G}\left(x_{i, \alpha}, y_{\alpha}\right)^{\varepsilon}-(1-\varepsilon) \mu_{\alpha}^{(1-2 \varepsilon)(n-2) / 2} \sum_{i=1}^{N} \mathscr{G}\left(x_{i, \alpha}, y_{\alpha}\right)^{1-\varepsilon} .
\end{aligned}
$$

Using (5-8) and (5-9), and since $0<\varepsilon<\frac{1}{2}$, it follows that

$$
\begin{aligned}
& \Delta_{g} \Psi_{\alpha, \varepsilon}\left(y_{\alpha}\right) \\
& \geq-(1-\varepsilon) \Psi_{\alpha, \varepsilon}\left(y_{\alpha}\right)-\gamma_{3} \varepsilon(1-\varepsilon) \Psi_{\alpha, \varepsilon}\left(y_{\alpha}\right)+\varepsilon(1-\varepsilon) \gamma_{2} \mu_{\alpha}^{(1-2 \varepsilon)(n-2) / 2} \sum_{i=1}^{N} d_{g}\left(x_{i, \alpha}, y_{\alpha}\right)^{-2} \varphi\left(x_{i, \alpha}, y_{\alpha}\right)^{1-\varepsilon} \\
& +\varepsilon(1-\varepsilon) \gamma_{2} \eta_{\alpha}(\delta) \sum_{i=1}^{N} d_{g}\left(x_{i, \alpha}, y_{\alpha}\right)^{-2} \varphi\left(x_{i, \alpha}, y_{\alpha}\right)^{\varepsilon} \\
& \geq-(1-\varepsilon)\left(1+\gamma_{3} \varepsilon\right) \Psi_{\alpha, \varepsilon}\left(y_{\alpha}\right)+\varepsilon(1-\varepsilon) \gamma_{2} \gamma_{1}^{\varepsilon-1} \mu_{\alpha}^{(1-2 \varepsilon)(n-2) / 2} r_{\alpha}\left(y_{\alpha}\right)^{-2-(n-2)(1-\varepsilon)} \\
& +\varepsilon(1-\varepsilon) \gamma_{2} \gamma_{1}^{-\varepsilon} \eta_{\alpha}(\delta) r_{\alpha}\left(y_{\alpha}\right)^{-2-(n-2) \varepsilon} .
\end{aligned}
$$


From (5-8) we obtain $\Psi_{\alpha, \varepsilon}\left(y_{\alpha}\right) \leq N \gamma_{1}^{1-\varepsilon} \mu_{\alpha}^{(1-2 \varepsilon)(n-2) / 2} r_{\alpha}\left(y_{\alpha}\right)^{-(n-2)(1-\varepsilon)}+N \gamma_{1}^{\varepsilon} \eta_{\alpha}(\delta) r_{\alpha}\left(y_{\alpha}\right)^{-(n-2) \varepsilon}$, and we can write

$$
r_{\alpha}\left(y_{\alpha}\right)^{2} \Delta_{g} \Psi_{\alpha, \varepsilon}\left(y_{\alpha}\right) \geq-(1-\varepsilon)\left(1+\gamma_{3} \varepsilon\right) r_{\alpha}\left(y_{\alpha}\right)^{2} \Psi_{\alpha, \varepsilon}\left(y_{\alpha}\right)+\frac{1}{N} \varepsilon(1-\varepsilon) \gamma_{2} \gamma_{1}^{2(\varepsilon-1)} \Psi_{\alpha, \varepsilon}\left(y_{\alpha}\right) .
$$

Coming back to (5-13), we thus get

$$
\frac{1}{N} \varepsilon(1-\varepsilon) \gamma_{2} \gamma_{1}^{2(\varepsilon-1)} \leq r_{\alpha}\left(y_{\alpha}\right)^{2}\left|u_{\alpha}\left(y_{\alpha}\right)\right|^{2^{\star}-2}+\delta^{2} p\|A\|_{\infty}+o(1)+(1-\varepsilon)\left(1+\gamma_{3} \varepsilon\right) \delta^{2}
$$

since we assumed that $r_{\alpha}\left(y_{\alpha}\right) \leq \delta$. By the last equation in (4-4) of Proposition 4.2 we can choose $\delta>0$ and $R>0$ so as to get a contradiction. Thus (5-11) is proved. Up to choosing $R$ a little bit larger, we deduce from the second equation in (4-5) of Proposition 4.2, and the definitions of $\mu_{\alpha}$ and $\eta_{\alpha}(\delta)$, that there exists $C>0$ such that

$$
\sup _{M \backslash \bigcup_{i=1}^{N} B_{x_{i, \alpha}}\left(R \mu_{i, \alpha}\right)} \frac{\sum_{i=1}^{p} u_{i, \alpha}}{\Psi_{\alpha, \varepsilon}} \leq C_{\varepsilon} .
$$

Using (5-8), we obtain the existence of $\delta_{\varepsilon}>0, R_{\varepsilon}>0$ and $C_{\varepsilon}>0$ such that

$$
\sum_{i=1}^{p} u_{i, \alpha}(x) \leq C_{\varepsilon}\left(\mu_{\alpha}^{(1-2 \varepsilon)(n-2) / 2} r_{\alpha}(x)^{(2-n)(1-\varepsilon)}+\eta_{\alpha}\left(\delta_{\varepsilon}\right) r_{\alpha}(x)^{(2-n) \varepsilon}\right)
$$

for all $\alpha$ and all $x \in M \backslash \bigcup_{i=1}^{N} B_{x_{i, \alpha}}\left(R_{\varepsilon} \mu_{i, \alpha}\right)$. This proves Step 1 .

Step 2. There exists $C_{0}>0$ such that $\left|u_{\alpha}(x)\right| \leq C_{0}\left(\mu_{\alpha}^{(n-2) / 2} \mathscr{D}_{\alpha}(x)^{2-n}+\left\|U_{\infty}\right\|_{\infty}\right)$ for all $\alpha$ and all $x \in M$.

Proof of Step 2. First we prove that there is $\delta>0$ small such that for any sequence $\left(y_{\alpha}\right)$ of points in $M$,

$$
\limsup _{\alpha \rightarrow+\infty} \frac{\left|u_{\alpha}\left(y_{\alpha}\right)\right|}{\mu_{\alpha}^{(n-2) / 2} \mathscr{D}_{\alpha}\left(y_{\alpha}\right)^{2-n}+\eta_{\alpha}(\delta)}<+\infty .
$$

By the definition of $\eta_{\alpha}(\delta)$, it is clear that (5-14) holds if $r_{\alpha}\left(y_{\alpha}\right) \geq \delta$. Now assume that $r_{\alpha}\left(y_{\alpha}\right)=O\left(\mu_{\alpha}\right)$. Then $\mathscr{D}_{\alpha}\left(y_{\alpha}\right)=O\left(\mu_{\alpha}\right)$. We can use the last equation in (4-4) of Proposition 4.2 to obtain

$$
\begin{aligned}
\mathscr{D}_{\alpha}\left(y_{\alpha}\right)^{2} \mu_{\alpha}^{-1}\left|\mathcal{U}_{\alpha}\left(y_{\alpha}\right)\right|^{2 / n-2} & =O\left(\mathscr{D}_{\alpha}\left(y_{\alpha}\right) \mu_{\alpha}^{-1}\right)+O\left(\sum_{i=1}^{N} \mathscr{D}_{\alpha}\left(y_{\alpha}\right)^{2} \mu_{\alpha}^{-1} \mu_{i, \alpha}^{-1}\left(1+\frac{d_{g}\left(x_{i, \alpha}, y_{\alpha}\right)^{2}}{n(n-2) \mu_{i, \alpha}^{2}}\right)^{-1}\right) \\
& =O(1),
\end{aligned}
$$

since $\mathscr{D}_{\alpha}\left(y_{\alpha}\right) \leq d_{g}\left(x_{i, \alpha}, y_{\alpha}\right)+\mu_{i, \alpha}$ for all $i \in\{1, \ldots, N\}$. In particular, (5-14) holds true also in this case. Thus we may assume from now on that

$$
r_{\alpha}\left(y_{\alpha}\right) \leq \delta \quad \text { and } \quad \frac{r_{\alpha}\left(y_{\alpha}\right)}{\mu_{\alpha}} \rightarrow+\infty \quad \text { as } \alpha \rightarrow+\infty
$$

We let $\lambda>1$ be such that $\lambda p\|A\|_{\infty} \notin S p\left(\Delta_{g}\right)$ and we let $G$ be the Green's function of $\Delta_{g}-\lambda p\|A\|_{\infty}$. Here again, there exist $C_{1}>1, C_{2}>0$ and $C_{3}>0$ such that

$$
\frac{1}{C_{1}} d_{g}(x, y)^{2-n}-C_{2} \leq G(x, y) \leq C_{1} d_{g}(x, y)^{2-n}
$$


and

$$
|\nabla G(x, y)|_{g} \leq C_{3} d_{g}(x, y)^{1-n}
$$

for all $x, y \in M, x \neq y$. We let $x_{0} \in \mathscr{Y}$ be such that $d_{g}\left(y_{\alpha}, x_{0}\right) \leq \delta+o(1)$; such an $x_{0}$ does exist thanks to (5-15). We choose $\delta>0$ such that

$$
d_{g}(x, y) \geq 4 \delta
$$

for all $x, y \in \mathscr{Y}, x \neq y$, and such that

$$
\delta \leq \frac{1}{4}\left(C_{1} C_{2}\right)^{-1 /(n-2)},
$$

where $C_{1}$ and $C_{2}$ are as in (5-16). We write with Green's representation formula that

$$
\begin{aligned}
& \sum_{i=1}^{p} u_{i, \alpha}\left(y_{\alpha}\right)=\int_{B_{x_{0}}(2 \delta)} G\left(y_{\alpha}, x\right)\left(\Delta_{g}\left(\sum_{i=1}^{p} u_{i, \alpha}\right)-\lambda p\|A\|_{\infty} \sum_{i=1}^{p} u_{i}^{\alpha}\right)(x) d v_{g}(x) \\
& \quad+\int_{\partial B_{x_{0}}(2 \delta)} G\left(y_{\alpha}, x\right) \partial_{\nu}\left(\sum_{i=1}^{p} u_{i, \alpha}\right)(x) d \sigma_{g}(x)-\int_{\partial B_{x_{0}}(2 \delta)} \partial_{\nu} G\left(y_{\alpha}, x\right)\left(\sum_{i=1}^{p} u_{i, \alpha}\right)(x) d \sigma_{g}(x) .
\end{aligned}
$$

Since $\lambda>1$, we get with (1-1) that

$$
\Delta_{g}\left(\sum_{i=1}^{p} u_{i, \alpha}\right)-\lambda p\|A\|_{\infty} \sum_{i=1}^{p} u_{i, \alpha} \leq\left|u_{\alpha}\right|^{2^{\star}-2} \sum_{i=1}^{p} u_{i, \alpha} .
$$

We have $G\left(y_{\alpha}, x\right) \geq 0$ in $B_{x_{0}}(2 \delta)$ for $\alpha$ large, thanks to (5-16) and (5-19). Thus we can write

$$
\begin{aligned}
\int_{B_{x_{0}}(2 \delta)} G\left(y_{\alpha}, x\right)\left(\Delta_{g}\left(\sum_{i=1}^{p} u_{i, \alpha}\right)-\lambda p\|A\|_{\infty} \sum_{i=1}^{p} u_{i, \alpha}\right)(x) d v_{g}(x) & \\
& \leq C_{1} \int_{M} d_{g}\left(y_{\alpha}, x\right)^{2-n}\left|u_{\alpha}(x)\right|^{2^{\star}-2} \sum_{i=1}^{p} u_{i, \alpha}(x) d v_{g}(x) .
\end{aligned}
$$

From (5-18), we also know that $d_{g}\left(x_{i, \alpha}, \partial B_{x_{0}}(2 \delta)\right) \geq \delta$ for all $i=1, \ldots, N$ and for $\alpha$ large so that we can control the boundary terms in (5-20) thanks to (5-4), (5-16) and (5-17). We thus obtain that

$$
\left|u_{\alpha}\left(y_{\alpha}\right)\right|=O\left(\eta_{\alpha}(\delta)\right)+O\left(\int_{M} d_{g}\left(y_{\alpha}, x\right)^{2-n}\left|u_{\alpha}(x)\right|^{2^{\star}-1} d v_{g}(x)\right) .
$$

We fix $0<\varepsilon<\frac{1}{n+2}$ and we let $R_{\varepsilon}>0, \delta_{\varepsilon}>0$ and $C_{\varepsilon}>0$ be given by Step 1. We write

$$
\begin{aligned}
& \int_{M} d_{g}\left(y_{\alpha}, x\right)^{2-n}\left|u_{\alpha}(x)\right|^{2^{\star}-1} d v_{g}(x) \\
& \leq \int_{M_{\alpha, \varepsilon}} d_{g}\left(y_{\alpha}, x\right)^{2-n}\left|u_{\alpha}(x)\right|^{2^{\star}-1} d v_{g}(x)+\sum_{i=1}^{p} \int_{B_{x_{i, \alpha}}\left(R_{\varepsilon} \mu_{\alpha}\right)} d_{g}\left(y_{\alpha}, x\right)^{2-n}\left|u_{\alpha}(x)\right|^{2^{\star}-1} d v_{g}(x),
\end{aligned}
$$

where $M_{\alpha, \varepsilon}=M \backslash \bigcup_{i=1}^{N} B_{x_{i, \alpha}}\left(R_{\varepsilon} \mu_{\alpha}\right)$. From (5-15) and Hölder's inequalities we obtain

$$
\int_{B_{x_{i, \alpha}}\left(R_{\varepsilon} \mu_{\alpha}\right)} d_{g}\left(y_{\alpha}, x\right)^{2-n}\left|\mathcal{U}_{\alpha}(x)\right|^{2^{\star}-1} d v_{g}(x)=O\left(\mu_{\alpha}^{(n-2) / 2} d_{g}\left(x_{i, \alpha}, y_{\alpha}\right)^{2-n}\right)
$$


for all $i \in\{1, \ldots, N\}$. Thus we get

$$
\begin{array}{rl}
\int_{M} d_{g}\left(y_{\alpha}, x\right)^{2-n}\left|\mathcal{U}_{\alpha}(x)\right|^{2^{\star}-1} & d v_{g}(x) \\
& \leq \int_{M_{\alpha, \varepsilon}} d_{g}\left(y_{\alpha}, x\right)^{2-n}\left|u_{\alpha}(x)\right|^{2^{\star}-1} d v_{g}(x)+O\left(\mu_{\alpha}^{(n-2) / 2} r_{\alpha}\left(y_{\alpha}\right)^{2-n}\right) .
\end{array}
$$

Using Step 1, we know that for any $x \in M_{\alpha, \varepsilon}$,

$$
\left|u_{\alpha}(x)\right|^{2^{\star}-1} \leq 2^{2^{\star}-2} C_{\varepsilon}^{2^{\star}-1}\left(\frac{\mu_{\alpha}^{(1-2 \varepsilon)(n+2) / 2}}{r_{\alpha}(x)^{(n+2)(1-\varepsilon)}}+\frac{\eta_{\alpha}\left(\delta_{\varepsilon}\right)^{2^{\star}-1}}{r_{\alpha}(x)^{(n+2) \varepsilon}}\right)
$$

so that

$$
\begin{aligned}
& \int_{M_{\alpha, \varepsilon}} d_{g}\left(y_{\alpha}, x\right)^{2-n}\left|\mathcal{U}_{\alpha}(x)\right|^{2^{\star}-1} d v_{g}(x) \\
& \leq 2^{2^{\star}-2} C_{\varepsilon}^{2^{\star}-1} \mu_{\alpha}^{(1-2 \varepsilon)(n+2) / 2} \int_{M_{\alpha, \varepsilon}} d_{g}\left(y_{\alpha}, x\right)^{2-n} r_{\alpha}(x)^{-(n+2)(1-\varepsilon)} d v_{g}(x) \\
& \quad+2^{2^{\star}-2} C_{\varepsilon}^{2^{\star}-1} \eta_{\alpha}\left(\delta_{\varepsilon}\right)^{2^{\star}-1} \int_{M_{\alpha, \varepsilon}} d_{g}\left(y_{\alpha}, x\right)^{2-n} r_{\alpha}(x)^{-(n+2) \varepsilon} d v_{g}(x) \\
& \leq 2^{2^{\star}-2} C_{\varepsilon}^{2^{\star}-1} \mu_{\alpha}^{(1-2 \varepsilon)(n+2) / 2} \sum_{i=1}^{N} \int_{M \backslash B_{x_{i, \alpha}}\left(R_{\varepsilon} \mu_{\alpha}\right)} d_{g}\left(y_{\alpha}, x\right)^{2-n} d_{g}\left(x_{i, \alpha}, x\right)^{-(n+2)(1-\varepsilon)} d v_{g}(x) \\
&+2^{2^{\star}-2} C_{\varepsilon}^{2^{\star}-1} \eta_{\alpha}\left(\delta_{\varepsilon}\right)^{2^{\star}-1} \sum_{i=1}^{N} \int_{M \backslash B_{x_{i, \alpha}}\left(R_{\varepsilon} \mu_{\alpha}\right)} d_{g}\left(y_{\alpha}, x\right)^{2-n} d_{g}\left(x_{i, \alpha}, x\right)^{-(n+2) \varepsilon} d v_{g}(x) .
\end{aligned}
$$

From (5-15), straightforward computations yield

$$
\int_{M_{\alpha, \varepsilon}} d_{g}\left(y_{\alpha}, x\right)^{2-n}\left|u_{\alpha}(x)\right|^{2^{\star}-1} d v_{g}(x)=O\left(\mu_{\alpha}^{(n-2) / 2} r_{\alpha}\left(y_{\alpha}\right)^{2-n}\right)+O\left(\eta_{\alpha}\left(\delta_{\varepsilon}\right)^{2^{\star}-1}\right) .
$$

Coming back to (5-23), using (5-3), we finally obtain that

$$
\int_{M} d_{g}\left(y_{\alpha}, x\right)^{2-n}\left|u_{\alpha}(x)\right|^{2^{\star}-1} d v_{g}(x)=O\left(\mu_{\alpha}^{(n-2) / 2} r_{\alpha}\left(y_{\alpha}\right)^{2-n}\right)+O\left(\eta_{\alpha}\left(\delta_{\varepsilon}\right)\right) .
$$

Coming back to (5-22), taking $0<\delta<\delta_{\varepsilon}$ such that (5-18) and (5-19) hold, we get (5-14) under assumption (5-15). In particular, if $\delta$ is chosen sufficiently small, (5-14) holds. Now we claim that if $u_{\infty} \equiv 0$, then

$$
\eta_{\alpha}(\delta)=O\left(\mu_{\alpha}^{(n-2) / 2}\right) .
$$

As a consequence of (5-14), there exists $C_{0}>0$ such that in any compact subset $K$ of $M \backslash \mathscr{Y}$,

$$
\left|u_{\alpha}(x)\right| \leq C_{0}\left(\mu_{\alpha}^{(n-2) / 2} C_{K}+\eta_{\alpha}(\delta)\right)
$$

for some $C_{K}>0$. If (5-24) were false, we would get by standard elliptic theory that

$$
\frac{u_{\alpha}}{\eta_{\alpha}(\delta)} \rightarrow H \text { in } C_{\mathrm{loc}}^{2}(M \backslash \mathscr{S}) \text { as } \alpha \rightarrow+\infty
$$


where $H$ satisfies $\Delta_{g} H+A H=0$ in $M \backslash \mathscr{Y}$ and $|H| \leq C_{0}$ in $M \backslash \mathscr{Y}$. This implies that $H$ is in the kernel of $\Delta_{g}+A$. Since all the components of $H$ are nonnegative and $H$ is not identically zero by the definition of $\eta_{\alpha}(\delta)$, this would contradict assumption $(\mathrm{H})$. In particular, (5-24) is proved. Noting that if $u_{\infty} \not \equiv 0$, then, by (5-3), $\eta_{\alpha}(\delta)=O\left(\left\|U_{\infty}\right\|_{\infty}\right)$, we get with (5-14) that Step 2 holds true.

Conclusion of the proof of Proposition 5.1. If $\boldsymbol{u}_{\infty} \equiv 0$, the proposition is a direct consequence of Step 2. Assume now that $u_{\infty} \not \equiv 0$. We let $\mathscr{H}$ be the Green's function of the Laplacian on $M$ normalized such that $\mathscr{H}(x, y) \geq 1$ for all $x, y \in M, x \neq y$. There exists $\Theta_{1}>1$ such that

$$
\frac{1}{\Theta_{1}} d_{g}(x, y)^{2-n} \leq \mathscr{H}(x, y) \leq \Theta_{1} d_{g}(x, y)^{2-n}
$$

for all $x, y \in M, x \neq y$. We let $\left(x_{\alpha}\right)$ be a sequence of points in $M$ and prove that

$$
\left|u_{\alpha}\left(x_{\alpha}\right)-u_{\infty}\left(x_{\alpha}\right)\right|=O\left(\mu_{\alpha}^{(n-2) / 2} \mathscr{D}_{\alpha}\left(x_{\alpha}\right)^{2-n}\right)+o(1) .
$$

If $\mathscr{D}_{\alpha}\left(x_{\alpha}\right)=O\left(\mu_{\alpha}\right)$, then (5-26) is a direct consequence of the last equation in (4-4) of Proposition 4.2. We may therefore assume that

$$
\frac{\mathscr{D}_{\alpha}\left(x_{\alpha}\right)}{\mu_{\alpha}} \rightarrow+\infty \quad \text { as } \alpha \rightarrow+\infty
$$

By standard elliptic theory,

$$
u_{\alpha} \rightarrow u_{\infty} \text { in } C_{\mathrm{loc}}^{2}(M \backslash \mathscr{S}) \text { as } \alpha \rightarrow+\infty,
$$

where $\mathscr{S}$ is as in (5-1). We write using Green's representation formula that

$$
\begin{aligned}
\sum_{i=1}^{p} u_{i, \alpha}\left(x_{\alpha}\right)-\sum_{i=1}^{p} u_{i, \infty}\left(x_{\alpha}\right)=\frac{1}{V_{g}} \sum_{i=1}^{p} \int_{M}\left(u_{i, \alpha}\left(x_{\alpha}\right)-u_{i, \infty}\left(x_{\alpha}\right)\right) d v_{g} & \\
& +\sum_{i=1}^{p} \int_{M} \mathscr{H}\left(x_{\alpha}, x\right) \Delta_{g}\left(u_{i, \alpha}-u_{i, \infty}\right)(x) d v_{g}(x)
\end{aligned}
$$

where $V_{g}$ is the volume of $(M, g)$, and the $u_{i, \infty}$ 's are the components of $u_{\infty}$. Then we get

$$
\sum_{i=1}^{p} u_{i, \alpha}\left(x_{\alpha}\right)-\sum_{i=1}^{p} u_{i, \infty}\left(x_{\alpha}\right)=\sum_{i=1}^{p} \int_{M} \mathcal{H}\left(x_{\alpha}, x\right) \Delta_{g}\left(u_{i, \alpha}-u_{i, \infty}\right)(x) d v_{g}(x)+o(1) .
$$

Thanks to (5-28) there exists $\delta_{\alpha}>0, \delta_{\alpha} \rightarrow 0$ as $\alpha \rightarrow+\infty$, such that, up to a subsequence,

$$
\left\|u_{\alpha}-u_{\infty}\right\|_{C^{2}\left(\left\{\mathscr{D}_{\alpha}>\delta_{\alpha}\right\}\right)}=o(1),
$$

where $\|u\|_{C^{2}}=\sum_{i=1}^{p}\left\|u_{i}\right\|_{C^{2}}$, and $\left\{\mathscr{D}_{\alpha}>\delta_{\alpha}\right\}$ is the subset of $M$ consisting of the $x \in M$ such that $\mathscr{D}_{\alpha}(x)>\delta_{\alpha}$. In particular, it follows from (5-25), (5-29) and (5-30) that

$$
\sum_{i=1}^{p} u_{i, \alpha}\left(x_{\alpha}\right)-\sum_{i=1}^{p} u_{i, \infty}\left(x_{\alpha}\right)=\sum_{i=1}^{p} \int_{\left\{\mathscr{D}_{\alpha}(x) \leq \delta_{\alpha}\right\}} \mathcal{H}\left(x_{\alpha}, x\right) \Delta_{g} u_{i, \alpha}(x) d v_{g}(x)+o(1) .
$$

The proof of (5-26) then follows the lines of the proof of Step 2, using the estimate of that step. This ends the proof of Proposition 5.1. 


\section{Strong pointwise estimates and sharp asymptotics}

We now turn to pointwise estimates and sharp asymptotics. Our main result in this section is this:

Proposition 6.1. Let $(M, g)$ be a smooth compact Riemannian manifold of dimension $n \geq 3, p \geq 1$ be an integer, and $\left(A_{\alpha}\right)_{\alpha}$ be a sequence of $C^{1}$ maps from $M$ to $M_{p}^{s}(\mathbb{R})$ such that $A_{\alpha} \rightarrow A$ in $C^{1}(M)$ as $\alpha \rightarrow+\infty$ for some $C^{1}$ map A from $M$ to $M_{p}^{s}(\mathbb{R})$ satisfying $(\mathrm{H})$. Let also $\left(\cup_{\alpha}\right)_{\alpha}$ be an arbitrary bounded sequence in $H^{1}(M)$ of nonnegative solutions of (1-1) such that $\left\|\cup_{\alpha}\right\|_{\infty} \rightarrow+\infty$ as $\alpha \rightarrow+\infty$. Up to passing to a subsequence on the $U_{\alpha}$ 's, there holds that for any sequence $\left(x_{\alpha}\right)_{\alpha}$ of points in $M$,

$$
\left|u_{\alpha}\left(x_{\alpha}\right)-u_{\infty}\left(x_{\alpha}\right)-\sum_{i=1}^{N} B_{i, \alpha}\left(x_{\alpha}\right) \Lambda_{i}\right|=\varepsilon_{\alpha}\left\|u_{\infty}\right\|_{\infty}+O\left(\mu_{\alpha}^{(n-2) / 2}\right)+o\left(\sum_{i=1}^{N} B_{i, \alpha}\left(x_{\alpha}\right)\right),
$$

where $\mathscr{B}_{\alpha}^{i}=B_{i, \alpha} \Lambda_{i}$ for all $\alpha$ and all $i$, where $U_{\infty}, N$, and the $\mathscr{B}_{\alpha}^{i}$ 's are as in Proposition 4.2, where $\varepsilon_{\alpha} \rightarrow 0$ as $\alpha \rightarrow+\infty$, and $\mu_{\alpha}=\max _{i} \mu_{i, \alpha}$ is the maximum weight of the weights of the $B_{i, \alpha}$ 's as in (5-6).

We prove Proposition 6.1 in several steps, based on induction on the following statement, defined for $1 \leq k \leq N+1$ :

There exists $C_{k}>0$ such that, up to a subsequence on the $u_{\alpha}$ 's, for any sequence $\left(x_{\alpha}\right)$ of points in $M$,

$$
\begin{aligned}
& \left|u_{\alpha}\left(x_{\alpha}\right)-u_{\infty}\left(x_{\alpha}\right)-\sum_{i=1}^{k-1} B_{i, \alpha}\left(x_{\alpha}\right) \Lambda_{i}\right| \\
& \quad \leq C_{k}\left(\mu_{\alpha}^{(n-2) / 2}+\chi_{k} \mu_{k, \alpha}^{(n-2) / 2} R_{k, \alpha}\left(x_{\alpha}\right)^{2-n}\right)+\varepsilon_{\alpha}\left\|u_{\infty}\right\|_{\infty}+o\left(\sum_{i=1}^{k-1} B_{i, \alpha}\left(x_{\alpha}\right)\right),
\end{aligned}
$$

where $\varepsilon_{\alpha} \rightarrow 0$ as $\alpha \rightarrow+\infty, \chi_{k}=1$ if $k \leq N$, and $\chi_{N+1}=0$.

Here we have reordered the blow-up points in such a way that $\mu_{\alpha}=\mu_{1, \alpha} \geq \mu_{2, \alpha} \geq \cdots \geq \mu_{N, \alpha}$, and we have defined

$$
\begin{aligned}
r_{i, \alpha}(x) & =\min _{i \leq j \leq N} d_{g}\left(x_{j, \alpha}, x\right), \\
R_{i, \alpha}(x) & =\min _{i \leq j \leq N}\left(d_{g}\left(x_{j, \alpha}, x\right)+\mu_{j, \alpha}\right) .
\end{aligned}
$$

We have $R_{1, \alpha}(x)=\mathscr{D}_{\alpha}(x)$ and $r_{1, \alpha}(x)=r_{\alpha}(x)$, where $\mathscr{D}_{\alpha}$ is as in Proposition 4.2 and $r_{\alpha}$ is as in (5-6).

We will refer to the whole indented statement above as $\left(\mathscr{I}_{k}\right)$, as well as the inequality so labeled. Clearly, Proposition 6.1 is equivalent to $\left(\mathscr{I}_{N+1}\right)$, while Proposition 5.1 implies $\left(\mathscr{I}_{1}\right)$.

We apply induction on $k$ to pass from $\left(\mathscr{I}_{1}\right)$ to $\left(\mathscr{I}_{N}\right)$, and then we use a slightly distinct argument to pass from $\left(\Phi_{N}\right)$ to $\left(\Phi_{N+1}\right)$. In the following, we fix $1 \leq \kappa \leq N-1$ and assume that $\left(\Phi_{\kappa}\right)$ holds true. We proceed in several steps, but first we fix some notation. As in the preceding section we let $\mathscr{G}$ be the Green's function of the operator $u \mapsto \Delta_{g} u+u$. Then (5-8) and (5-9) hold. We fix $0<\varepsilon<1 /(n+2)$ and fix $R_{0}$ as in (5-5). For any $1 \leq i \leq \kappa$, we define

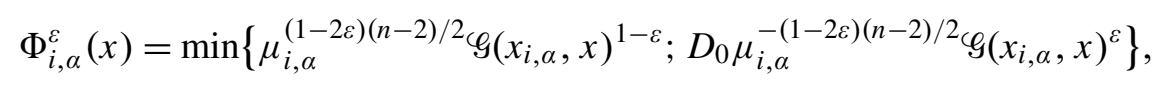

where $x \in M \backslash\left\{x_{i, \alpha}\right\}$,

$$
D_{0}=\gamma_{1}^{2 \varepsilon-1}\left(4 R_{0}\right)^{(2-n)(1-2 \varepsilon)},
$$


and $\gamma_{1}$ is as in (5-8). This choice of $D_{0}$, together with (5-8), implies that

$$
\Phi_{i, \alpha}^{\varepsilon}(x)=D_{0} \mu_{i, \alpha}^{-(1-2 \varepsilon)(n-2) / 2} \mathscr{G}\left(x_{i, \alpha}, x\right)^{\varepsilon},
$$

if $d_{g}\left(x_{i, \alpha}, x\right) \leq 2 R_{0} \mu_{i, \alpha}$. We also let

and

$$
\varphi_{\alpha}^{\varepsilon}(x)=\max \left\{\left\|u_{\infty}\right\|_{\infty} ; \mu_{\alpha}^{(1-2 \varepsilon)(n-2) / 2}\right\} \sum_{i=1}^{N} \mathscr{G}\left(x_{i, \alpha}, x\right)^{\varepsilon}
$$

For $1 \leq i \leq \kappa$, we set

$$
\Psi_{\alpha}^{\varepsilon}(x)=\sum_{i=\kappa+1}^{N} \mathscr{G}\left(x_{i, \alpha}, x\right)^{1-\varepsilon} \text {. }
$$

$$
\Omega_{i, \alpha}^{\varepsilon}=\left\{x \in M \text { s.t. } \Phi_{i, \alpha}^{\varepsilon}(x) \geq \Phi_{j, \alpha}^{\varepsilon}(x) \text { for all } 1 \leq j \leq \kappa\right\} .
$$

We also set

$$
D(\varepsilon)=\frac{\gamma_{2} \varepsilon(1-\varepsilon)}{2 N},
$$

where $\gamma_{2}$ is as in (5-8), and we define $v_{k, \alpha}$ by

$$
v_{\kappa, \alpha}^{(1-2 \varepsilon)(n-2) / 2}=\max \left\{\mu_{\kappa+1, \alpha}^{(1-2 \varepsilon)(n-2) / 2} ; \max _{1 \leq i \leq \kappa} \sup _{\tilde{\Omega}_{i, \alpha}^{\varepsilon}} \frac{\Phi_{i, \alpha}^{\varepsilon}(x)}{\Psi_{\alpha}^{\varepsilon}(x)}\right\},
$$

where

$$
\tilde{\Omega}_{i, \alpha}^{\varepsilon}=\left\{x \in \Omega_{i, \alpha}^{\varepsilon} \text { s.t. } d_{g}\left(x_{i, \alpha}, x\right)^{2} \mathscr{Q}_{\kappa, \alpha}(x)^{2^{\star}-2} \geq D(\varepsilon)\right\},
$$

and $\mathscr{2}_{\kappa, \alpha}(x)=\left|\mathcal{U}_{\alpha}(x)-\mathcal{U}_{\infty}(x)-\sum_{i=1}^{\kappa} B_{i, \alpha}(x) \Lambda_{i}\right|$. By convention, the suprema in (6-9) are $-\infty$ if the sets $\tilde{\Omega}_{i, \alpha}^{\varepsilon}$ are empty. We can now start the proof of Proposition 6.1.

Step 1. $v_{\kappa, \alpha}=O\left(\mu_{\kappa, \alpha}\right)$.

Proof of Step 1. We let $y_{\alpha} \in \tilde{\Omega}_{i, \alpha}^{\varepsilon}$ and assume that

$$
v_{\kappa, \alpha}^{1-2 \varepsilon} \Psi_{\alpha}^{\varepsilon}\left(y_{\alpha}\right)^{2 /(n-2)}=\Phi_{i, \alpha}^{\varepsilon}\left(y_{\alpha}\right)^{2 /(n-2)} .
$$

This and (5-8) imply that

$$
v_{\kappa, \alpha}^{1-2 \varepsilon}=O\left(R_{\kappa+1, \alpha}\left(y_{\alpha}\right)^{2(1-\varepsilon)} \Phi_{i, \alpha}^{\varepsilon}\left(y_{\alpha}\right)^{2 /(n-2)}\right) .
$$

Since $\left(\mathscr{I}_{k}\right)$ holds and $y_{\alpha} \in \tilde{\Omega}_{i, \alpha}^{\varepsilon}$, we also have

$$
D(\varepsilon) \leq o(1)+o\left(\sum_{j=1}^{\kappa-1} d_{g}\left(x_{i, \alpha}, y_{\alpha}\right)^{2} B_{j, \alpha}\left(y_{\alpha}\right)^{2^{\star}-2}\right)+O\left(\mu_{\kappa, \alpha}^{2} d_{g}\left(x_{i, \alpha}, y_{\alpha}\right)^{2} R_{\kappa, \alpha}\left(y_{\alpha}\right)^{-4}\right) .
$$

Since $y_{\alpha} \in \tilde{\Omega}_{i, \alpha}^{\varepsilon}$ and $\tilde{\Omega}_{i, \alpha}^{\varepsilon} \subset \Omega_{i, \alpha}^{\varepsilon}$, we can write

and we thus get

$$
\sum_{j=1}^{\kappa-1} d_{g}\left(x_{i, \alpha}, y_{\alpha}\right)^{2} B_{j, \alpha}\left(y_{\alpha}\right)^{2^{\star}-2}=O(1)
$$

$$
R_{\kappa, \alpha}\left(y_{\alpha}\right)^{2}=O\left(\mu_{\kappa, \alpha} d_{g}\left(x_{i, \alpha}, y_{\alpha}\right)\right) .
$$

If $R_{\kappa+1, \alpha}\left(y_{\alpha}\right)=O\left(R_{\kappa, \alpha}\left(y_{\alpha}\right)\right)$, we get from (6-11) and (6-12) that

$$
v_{\kappa, \alpha}^{1-2 \varepsilon}=O\left(\mu_{\kappa, \alpha}^{1-\varepsilon} d_{g}\left(x_{i, \alpha}, y_{\alpha}\right)^{1-\varepsilon} \Phi_{i, \alpha}^{\varepsilon}\left(y_{\alpha}\right)^{2 /(n-2)}\right)=O\left(\mu_{\kappa, \alpha}^{1-\varepsilon} \mu_{i, \alpha}^{-\varepsilon}\right)=O\left(\mu_{\kappa, \alpha}^{1-2 \varepsilon}\right),
$$


and Step 1 is proved. Assume now that $R_{\kappa, \alpha}\left(y_{\alpha}\right)=o\left(R_{\kappa+1, \alpha}\left(y_{\alpha}\right)\right)$. Then (6-12) becomes

$$
\left(d_{g}\left(x_{\kappa, \alpha}, y_{\alpha}\right)+\mu_{\kappa, \alpha}\right)^{2}=O\left(\mu_{\kappa, \alpha} d_{g}\left(x_{i, \alpha}, y_{\alpha}\right)\right) \text {. }
$$

If $i=\kappa$ we obtain $d_{g}\left(x_{i, \alpha}, y_{\alpha}\right)=O\left(\mu_{i, \alpha}\right)$; using the last equation in (4-4), and since $\mu_{i, \alpha}=o\left(R_{\kappa+1, \alpha}\left(y_{\alpha}\right)\right)$, we obtain that

$$
d_{g}\left(x_{i, \alpha}, y_{\alpha}\right)^{2}\left|u_{\alpha}\left(y_{\alpha}\right)-u_{\infty}\left(y_{\alpha}\right)-\sum_{j=1}^{\kappa} B_{j, \alpha}\left(y_{\alpha}\right) \Lambda_{j}\right|^{2^{\star}-2} \rightarrow 0 \quad \text { as } \alpha \rightarrow+\infty .
$$

This contradicts the fact that $y_{\alpha} \in \tilde{\Omega}_{i, \alpha}^{\varepsilon}$. Thus we must have $1 \leq i \leq \kappa-1$. Since $\Phi_{i, \alpha}^{\varepsilon}\left(y_{\alpha}\right) \geq \Phi_{\kappa, \alpha}^{\varepsilon}\left(y_{\alpha}\right)$, because of (5-8), we can write

$\mu_{\kappa, \alpha}^{1-2 \varepsilon} d_{g}\left(x_{\kappa, \alpha}, y_{\alpha}\right)^{-2 \varepsilon}\left(\mu_{\kappa, \alpha}+d_{g}\left(x_{\kappa, \alpha}, y_{\alpha}\right)\right)^{-2(1-2 \varepsilon)}=O\left(\mu_{i, \alpha}^{1-2 \varepsilon} d_{g}\left(x_{i, \alpha}, y_{\alpha}\right)^{-2 \varepsilon}\left(\mu_{i, \alpha}+d_{g}\left(x_{i, \alpha}, y_{\alpha}\right)\right)^{-2(1-2 \varepsilon)}\right)$.

In particular we obtain with (6-13) that

$$
\left(\mu_{i, \alpha}+d_{g}\left(x_{i, \alpha}, y_{\alpha}\right)\right)^{1-\varepsilon}=O\left(\mu_{\kappa, \alpha}^{\varepsilon} \mu_{i, \alpha}^{1-2 \varepsilon}\right) .
$$

Since $\mu_{k, \alpha} \leq \mu_{i, \alpha}$, this implies that $d_{g}\left(x_{i, \alpha}, y_{\alpha}\right)=O\left(\mu_{i, \alpha}\right)$. We also get $\mu_{i, \alpha}=O\left(\mu_{\kappa, \alpha}\right)$. Then we obtain with (6-13) that $d_{g}\left(x_{\kappa, \alpha}, y_{\alpha}\right)=O\left(\mu_{i, \alpha}\right)$, and this contradicts the first equation in (4-5) of Proposition 4.2. Step 1 is proved.

Step 2. There exists $C_{\varepsilon}>0$ such that

$\left|u_{\alpha}(x)\right| \leq C_{\varepsilon}\left(\sum_{i=1}^{\kappa} \Phi_{i, \alpha}^{\varepsilon}(x)+v_{\kappa, \alpha}^{(1-2 \varepsilon)(n-2) / 2} r_{\kappa+1, \alpha}(x)^{(2-n)(1-\varepsilon)}+\max \left\{\left\|u_{\infty}\right\|_{\infty} ; \mu_{\alpha}^{(1-2 \varepsilon)(n-2) / 2}\right\} r_{\alpha}(x)^{(2-n) \varepsilon}\right)$ for all $x \in M \backslash \bigcup_{i=\kappa+1}^{N} B_{x_{i, \alpha}}\left(R_{0} \mu_{i, \alpha}\right)$.

Proof of Step 2. We let $y_{\alpha} \in M \backslash \bigcup_{i=\kappa+1}^{N} B_{x_{i, \alpha}}\left(R_{0} \mu_{i, \alpha}\right)$ be such that

$$
\frac{\sum_{i=1}^{p} u_{i, \alpha}}{\sum_{i=1}^{\kappa} \Phi_{i, \alpha}^{\varepsilon}+v_{\kappa, \alpha}^{(1-2 \varepsilon)(n-2) / 2} \Psi_{\alpha, \varepsilon}+\varphi_{\alpha}^{\varepsilon}}\left(y_{\alpha}\right)=\sup _{M \backslash \bigcup_{i=\kappa+1}^{N} B_{x_{i, \alpha}}\left(R_{\varepsilon} \mu_{i, \alpha}\right)} \frac{\sum_{i=1}^{p} u_{i, \alpha}}{\sum_{i=1}^{\kappa} \Phi_{i, \alpha}^{\varepsilon}+v_{\kappa, \alpha}^{(1-2 \varepsilon)(n-2) / 2} \Psi_{\alpha, \varepsilon}+\varphi_{\alpha}^{\varepsilon}},
$$

and we assume by contradiction that

$$
\frac{\sum_{i=1}^{p} u_{i}^{\alpha}}{\sum_{i=1}^{\kappa} \Phi_{i, \alpha}^{\varepsilon}+v_{\kappa, \alpha}^{(1-2 \varepsilon)(n-2) / 2} \Psi_{\alpha, \varepsilon}+\varphi_{\alpha}^{\varepsilon}}\left(y_{\alpha}\right) \rightarrow+\infty \quad \text { as } \alpha \rightarrow+\infty .
$$

From $\left(\mathscr{I}_{\kappa}\right)$ and (6-15) we get

$$
r_{\alpha}\left(y_{\alpha}\right) \rightarrow 0 \quad \text { as } \alpha \rightarrow+\infty .
$$

We also have, using the second equation in (4-5),

$$
\frac{d_{g}\left(x_{j, \alpha}, y_{\alpha}\right)}{\mu_{j, \alpha}} \rightarrow+\infty
$$

as $\alpha \rightarrow+\infty$ for all $\kappa+1 \leq j \leq N$. Here we used the fact that, by (6-9), $v_{\kappa, \alpha} \geq \mu_{\kappa+1, \alpha}$. Thanks to (6-15) and the second equation in (4-5), we also know that, for any $1 \leq j \leq \kappa$, either

$$
d_{g}\left(x_{j, \alpha}, y_{\alpha}\right) \leq R_{0} \mu_{j, \alpha} \quad \text { or } \quad \frac{d_{g}\left(x_{j, \alpha}, y_{\alpha}\right)}{\mu_{j, \alpha}} \rightarrow+\infty \quad \text { as } \alpha \rightarrow+\infty \text {. }
$$


In particular, thanks to (6-14) we can write

$$
\frac{\Delta_{g} \sum_{i=1}^{p} u_{i, \alpha}}{\sum_{i=1}^{p} u_{i, \alpha}}\left(y_{\alpha}\right) \geq \frac{\Delta_{g}\left(\sum_{i=1}^{\kappa} \Phi_{i, \alpha}^{\varepsilon}+v_{\kappa, \alpha}^{(1-2 \varepsilon)(n-2) / 2} \Psi_{\alpha, \varepsilon}+\varphi_{\alpha}^{\varepsilon}\right)}{\sum_{i=1}^{\kappa} \Phi_{i, \alpha}^{\varepsilon}+v_{\kappa, \alpha}^{(1-2 \varepsilon)(n-2) / 2} \Psi_{\alpha, \varepsilon}+\varphi_{\alpha}^{\varepsilon}}\left(y_{\alpha}\right) .
$$

From (1-1), (5-8), and (5-9), we then get

$$
\begin{aligned}
0 \geq \sum_{i=1}^{\kappa}\left(d_{g}\left(x_{i, \alpha}, y_{\alpha}\right)^{-2}-\frac{\left|u_{\alpha}\left(y_{\alpha}\right)\right|^{2^{\star}-2}}{\gamma_{2} \varepsilon(1-\varepsilon)}\right. & \left.-A_{\varepsilon}\right) \Phi_{i, \alpha}^{\varepsilon}\left(y_{\alpha}\right) \\
& +\left(\frac{r_{\kappa+1, \alpha}\left(y_{\alpha}\right)^{-2}}{N \gamma_{1}^{2(1-\varepsilon)}}-\frac{\left|u_{\alpha}\left(y_{\alpha}\right)\right|^{2^{\star}-2}}{\gamma_{2} \varepsilon(1-\varepsilon)}-A_{\varepsilon}\right) v_{\kappa, \alpha}^{(1-2 \varepsilon)(n-2) / 2} \Psi_{\alpha, \varepsilon}\left(y_{\alpha}\right) \\
& +\left(\frac{r_{\alpha}\left(y_{\alpha}\right)^{-2}}{N \gamma_{1}^{2 \varepsilon}}-\frac{\left|u_{\alpha}\left(y_{\alpha}\right)\right|^{2^{\star}-2}}{\gamma_{2} \varepsilon(1-\varepsilon)}-A_{\varepsilon}\right) \varphi_{\alpha, \varepsilon}\left(y_{\alpha}\right)
\end{aligned}
$$

where

$$
A_{\varepsilon}=\frac{p\left\|A_{\alpha}\right\|_{\infty}+\left(1+\gamma_{3} \varepsilon\right)(1-\varepsilon)}{\gamma_{2} \varepsilon(1-\varepsilon)} .
$$

We let in the following $1 \leq i \leq \kappa$ be such that $y_{\alpha} \in \Omega_{i, \alpha}^{\varepsilon}$. Then we deduce from (6-20) that

$$
\begin{aligned}
0 \geq\left(d_{g}\left(x_{i, \alpha}, y_{\alpha}\right)^{-2}-\frac{\kappa}{\gamma_{2} \varepsilon(1-\varepsilon)}\left|u_{\alpha}\left(y_{\alpha}\right)\right|^{2^{\star}-2}-\kappa A_{\varepsilon}\right) \Phi_{i, \alpha}^{\varepsilon}\left(y_{\alpha}\right) & \\
& +\left(\frac{r_{\kappa+1, \alpha}\left(y_{\alpha}\right)^{-2}}{N \gamma_{1}^{2(1-\varepsilon)}}-\frac{\left|u_{\alpha}\left(y_{\alpha}\right)\right|^{2^{\star}-2}}{\gamma_{2} \varepsilon(1-\varepsilon)}-A_{\varepsilon}\right) \nu_{\kappa, \alpha}^{(1-2 \varepsilon)(n-2) / 2} \Psi_{\alpha, \varepsilon}\left(y_{\alpha}\right) \\
& +\left(\frac{r_{\alpha}\left(y_{\alpha}\right)^{-2}}{N \gamma_{1}^{2 \varepsilon}}-\frac{\left|u_{\alpha}\left(y_{\alpha}\right)\right|^{2^{\star}-2}}{\gamma_{2} \varepsilon(1-\varepsilon)}-A_{\varepsilon}\right) \varphi_{\alpha, \varepsilon}\left(y_{\alpha}\right)
\end{aligned}
$$

From (6-15), we know that

$$
\left\|u_{\infty}\right\|_{\infty}=o\left(\left|u_{\alpha}\left(y_{\alpha}\right)\right|\right) \text { and } \mu_{\alpha}^{(n-2) / 2}=o\left(\left|u_{\alpha}\left(y_{\alpha}\right)\right|\right),
$$

and that

$$
B_{j, \alpha}\left(y_{\alpha}\right)=o\left(\left|u_{\alpha}\left(y_{\alpha}\right)\right|\right)
$$

for all $1 \leq j \leq \kappa$ since

$$
B_{j, \alpha}\left(y_{\alpha}\right)=O\left(\Phi_{j, \alpha}^{\varepsilon}\left(y_{\alpha}\right)\right)
$$

for all $1 \leq j \leq \kappa$. From (6-17), we have

$$
R_{\kappa+1, \alpha}\left(y_{\alpha}\right)^{2} B_{j, \alpha}\left(y_{\alpha}\right)^{2^{\star}-2}=o(1)
$$

for all $\kappa+1 \leq j \leq N$. Thus we can deduce from the last equation in (4-4) of Proposition 4.2 together with (6-22), (6-23), and (6-25), that

$$
\mathscr{D}_{\alpha}\left(y_{\alpha}\right)^{2}\left|u_{\alpha}\left(y_{\alpha}\right)\right|^{2^{\star}-2}=o(1) .
$$


Using (6-16) and (6-26), we can transform (6-21) into

$$
\begin{aligned}
0 \geq\left(d_{g}\left(x_{i, \alpha}, y_{\alpha}\right)^{-2}-\frac{\kappa}{\gamma_{2} \varepsilon(1-\varepsilon)}\left|U_{\alpha}\left(y_{\alpha}\right)\right|^{2^{\star}-2}-\kappa A_{\varepsilon}\right) \Phi_{i, \alpha}^{\varepsilon}\left(y_{\alpha}\right) & \\
& +\left(\frac{r_{\kappa+1, \alpha}\left(y_{\alpha}\right)^{-2}}{N \gamma_{1}^{2(1-\varepsilon)}}-\frac{\left|u_{\alpha}\left(y_{\alpha}\right)\right|^{2^{\star}-2}}{\gamma_{2} \varepsilon(1-\varepsilon)}-A_{\varepsilon}\right) \nu_{\kappa, \alpha}^{(1-2 \varepsilon)(n-2) / 2} \Psi_{\alpha, \varepsilon}\left(y_{\alpha}\right) \\
& +\left(\frac{1}{N \gamma_{1}^{2 \varepsilon}}+o(1)\right) r_{\alpha}\left(y_{\alpha}\right)^{-2} \varphi_{\alpha, \varepsilon}\left(y_{\alpha}\right)
\end{aligned}
$$

Since $\left(\Phi_{\kappa}\right)$ holds true, we can prove with (6-22) and (6-23) that

$$
\left|u_{\alpha}\left(y_{\alpha}\right)\right|^{2^{\star}-2}=O\left(\mu_{\kappa, \alpha}^{2} R_{\kappa+1, \alpha}\left(y_{\alpha}\right)^{-4}\right) .
$$

This implies that

$$
R_{\kappa+1, \alpha}\left(y_{\alpha}\right)^{2}\left|u_{\alpha}\left(y_{\alpha}\right)\right|^{2^{\star}-2} \rightarrow 0 \quad \text { as } \alpha \rightarrow+\infty .
$$

Indeed, if it is not the case, we would have from (6-28) that

$$
R_{\kappa+1, \alpha}\left(y_{\alpha}\right)=O\left(\mu_{k, \alpha}\right)
$$

and thanks to (6-26) that there exists $j \in\{1, \ldots, \kappa\}$ such that

$$
d_{g}\left(x_{j, \alpha}, y_{\alpha}\right)+\mu_{j, \alpha}=o\left(R_{\kappa+1, \alpha}\left(y_{\alpha}\right)\right) .
$$

In particular, we get a contradiction since $\mu_{j, \alpha} \geq \mu_{\kappa, \alpha}$. As a remark, (6-28) also implies that

$$
R_{\kappa+1, \alpha}\left(y_{\alpha}\right) \rightarrow 0 \quad \text { as } \alpha \rightarrow+\infty,
$$

due to (6-23). Now, thanks to (6-29) and (6-30), we deduce from (6-27) that

$$
\begin{aligned}
0 \geq\left(d_{g}\left(x_{i, \alpha}, y_{\alpha}\right)^{-2}-\frac{\kappa}{\gamma_{2} \varepsilon(1-\varepsilon)}\left|u_{\alpha}\left(y_{\alpha}\right)\right|^{2^{\star}-2}-\kappa A_{\varepsilon}\right) \Phi_{i, \alpha}^{\varepsilon}\left(y_{\alpha}\right) & \\
& +\left(\frac{1}{N \gamma_{1}^{2(1-\varepsilon)}}+o(1)\right) \nu_{\kappa, \alpha}^{(1-2 \varepsilon)(n-2) / 2} r_{\kappa+1, \alpha}\left(y_{\alpha}\right)^{-2} \Psi_{\alpha, \varepsilon}\left(y_{\alpha}\right) \\
& +\left(\frac{1}{N \gamma_{1}^{2 \varepsilon}}+o(1)\right) r_{\alpha}\left(y_{\alpha}\right)^{-2} \varphi_{\alpha, \varepsilon}\left(y_{\alpha}\right)
\end{aligned}
$$

If $y_{\alpha} \notin \tilde{\Omega}_{i, \alpha}^{\varepsilon}$, we transform (6-31) into

$$
\begin{aligned}
0 \geq(1+ & \left.o(1)-\frac{\kappa D(\varepsilon)}{\gamma_{2} \varepsilon(1-\varepsilon)}-\kappa A_{\varepsilon} d_{g}\left(x_{i, \alpha}, y_{\alpha}\right)^{2}\right) d_{g}\left(x_{i, \alpha}, y_{\alpha}\right)^{-2} \Phi_{i, \alpha}^{\varepsilon}\left(y_{\alpha}\right) \\
& +\left(\frac{1}{N \gamma_{1}^{2(1-\varepsilon)}}+o(1)\right) v_{\kappa, \alpha}^{(1-2 \varepsilon)(n-2) / 2} r_{\kappa+1, \alpha}\left(y_{\alpha}\right)^{-2} \Psi_{\alpha, \varepsilon}\left(y_{\alpha}\right)+\left(\frac{1}{N \gamma_{1}^{2 \varepsilon}}+o(1)\right) r_{\alpha}\left(y_{\alpha}\right)^{-2} \varphi_{\alpha, \varepsilon}\left(y_{\alpha}\right)
\end{aligned}
$$

by using (6-22) and (6-23). This leads to

$$
\left(\frac{1}{N \gamma_{1}^{2 \varepsilon}}+o(1)\right) r_{\alpha}\left(y_{\alpha}\right)^{-2} \varphi_{\alpha, \varepsilon}\left(y_{\alpha}\right)=O\left(\mu_{i, \alpha}^{(1-2 \varepsilon)(n-2) / 2}\right),
$$

thanks to our choice of $D(\varepsilon)$. From (5-8), (6-16), and the definition of $\varphi_{\alpha, \varepsilon}$, we clearly get a contradiction. 
Thus $y_{\alpha} \in \tilde{\Omega}_{i, \alpha}^{\varepsilon}$. Coming back to (6-31), we obtain in this situation that

$$
\left(\frac{1}{N \gamma_{1}^{2(1-\varepsilon)}}+o(1)\right) v_{\kappa, \alpha}^{(1-2 \varepsilon)(n-2) / 2} \Psi_{\alpha, \varepsilon}\left(y_{\alpha}\right) \leq\left(\frac{\kappa}{\gamma_{2} \varepsilon(1-\varepsilon)}\left|u_{\alpha}\left(y_{\alpha}\right)\right|^{2^{\star}-2}+\kappa A_{\varepsilon}\right) r_{\kappa+1, \alpha}\left(y_{\alpha}\right)^{2} \Phi_{i, \alpha}^{\varepsilon}\left(y_{\alpha}\right) \text {. }
$$

Using (6-29), (6-30), and the definition of $v_{\kappa, \alpha}$, this leads to

$$
\left(\frac{1}{N \gamma_{1}^{2(1-\varepsilon)}}+o(1)\right) v_{\kappa, \alpha}^{(1-2 \varepsilon)(n-2) / 2} \Psi_{\alpha, \varepsilon}\left(y_{\alpha}\right)=o\left(\Phi_{i, \alpha}^{\varepsilon}\left(y_{\alpha}\right)\right)=o\left(v_{\kappa, \alpha}^{(1-2 \varepsilon)(n-2) / 2} \Psi_{\alpha, \varepsilon}\left(y_{\alpha}\right)\right)
$$

and this is again a contradiction. Thus (6-15) cannot hold true and we get the equation in Step 2 from (5-8). This ends the proof of Step 2.

Step 3. There exists $C_{0}>0$ such that

$$
\left|u_{\alpha}(x)\right| \leq C_{0}\left(\sum_{i=1}^{\kappa} B_{i, \alpha}(x)+\left\|u_{\infty}\right\|_{\infty}+v_{\kappa, \alpha}^{(n-2) / 2} R_{\kappa+1, \alpha}(x)^{2-n}\right) \text { for all } x \in M \text { and all } \alpha>0 .
$$

Proof of Step 3. We let $\left(y_{\alpha}\right)$ be a sequence of points in $M$ and we aim to prove that

$$
\limsup _{\alpha \rightarrow+\infty} \frac{\left|U_{\alpha}\left(y_{\alpha}\right)\right|}{\sum_{i=1}^{\kappa} B_{i, \alpha}\left(y_{\alpha}\right)+\left\|U_{\infty}\right\|_{\infty}+v_{\kappa, \alpha}^{(n-2) / 2} R_{\kappa+1, \alpha}\left(y_{\alpha}\right)^{2-n}}<+\infty .
$$

Since $\left(\Phi_{\kappa}\right)$ holds true, it is clear that (6-32) also holds true as soon as

$$
\mu_{\kappa, \alpha}^{(n-2) / 2} R_{\kappa+1, \alpha}\left(y_{\alpha}\right)^{2-n}=O\left(B_{i, \alpha}\left(y_{\alpha}\right)\right)
$$

for some $1 \leq i \leq \kappa$. By contradiction we assume in what follows that (6-32) does not hold true. Thus we assume from now on that

$$
R_{\kappa+1, \alpha}\left(y_{\alpha}\right)^{2}=o\left(\mu_{i, \alpha} \mu_{\kappa, \alpha}\right)+o\left(\frac{\mu_{\kappa, \alpha}}{\mu_{i, \alpha}} d_{g}\left(x_{i, \alpha}, y_{\alpha}\right)^{2}\right)
$$

for all $1 \leq i \leq \kappa$. This implies in particular that

$$
R_{\kappa+1, \alpha}\left(y_{\alpha}\right) \rightarrow 0 \text { as } \alpha \rightarrow+\infty .
$$

Thanks to the last equation in (4-4) and to (6-33), we can assume that

$$
R_{\alpha}\left(y_{\alpha}\right)=R_{\kappa+1, \alpha}\left(y_{\alpha}\right) \quad \text { and } \quad \frac{R_{\kappa+1, \alpha}\left(y_{\alpha}\right)}{v_{\kappa, \alpha}} \rightarrow+\infty \quad \text { as } \alpha \rightarrow+\infty .
$$

Indeed, otherwise, (6-32) holds true. We let $\lambda>1$ be such that $\lambda p\|A\|_{\infty} \notin \operatorname{Sp}\left(\Delta_{g}\right)$, where $\operatorname{Sp}\left(\Delta_{g}\right)$ is the spectrum of $\Delta_{g}$, and let $G$ be the Green's function of $\mathfrak{L}_{g}=\Delta_{g}-\lambda p\|A\|_{\infty}$. There exist $C_{1}>1, C_{2}>0$ and $C_{3}>0$ such that

$$
\frac{1}{C_{1}} d_{g}(x, y)^{2-n}-C_{2} \leq G(x, y) \leq C_{1} d_{g}(x, y)^{2-n}
$$

and

$$
|\nabla G(x, y)|_{g} \leq C_{3} d_{g}(x, y)^{1-n}
$$

for all $x, y \in M, x \neq y$. We let $x_{0} \in \mathscr{Y}$ be such that $d_{g}\left(y_{\alpha}, x_{0}\right) \leq \delta+o(1)$; such an $x_{0}$ does exist thanks to (6-34). We choose $\delta>0$ such that

$$
d_{g}(x, y) \geq 4 \delta
$$


for all distinct $x, y \in \mathscr{Y}$, and such that

$$
\delta \leq \frac{1}{4}\left(C_{1} C_{2}\right)^{-1 /(n-2)}
$$

where $C_{1}$ and $C_{2}$ are as in (6-36). We write with Green's representation formula that

$$
\begin{aligned}
& \sum_{i=1}^{p} u_{i, \alpha}\left(y_{\alpha}\right)=\int_{B_{x_{0}}(2 \delta)} G\left(y_{\alpha}, x\right) \mathfrak{L}_{g}\left(\sum_{i=1}^{p} u_{i, \alpha}\right)(x) d v_{g}(x) \\
& \quad+\int_{\partial B_{x_{0}}(2 \delta)} G\left(y_{\alpha}, x\right) \partial_{\nu}\left(\sum_{i=1}^{p} u_{i, \alpha}\right)(x) d \sigma_{g}(x)-\int_{\partial B_{x_{0}}(2 \delta)} \partial_{\nu} G\left(y_{\alpha}, x\right)\left(\sum_{i=1}^{p} u_{i, \alpha}\right)(x) d \sigma_{g}(x) .
\end{aligned}
$$

Since $\lambda>1$, we get with (1-1) that

$$
\mathfrak{L}_{g}\left(\sum_{i=1}^{p} u_{i, \alpha}\right) \leq\left|u_{\alpha}\right|^{2^{\star}-2} \sum_{i=1}^{p} u_{i, \alpha} .
$$

We have $G\left(y_{\alpha}, x\right) \geq 0$ in $B_{x_{0}}(2 \delta)$ for $\alpha$ large by (6-36) and (6-39). Thus we can write

$$
\int_{B_{x_{0}}(2 \delta)} G\left(y_{\alpha}, x\right) \mathfrak{L}_{g}\left(\sum_{i=1}^{p} u_{i, \alpha}\right)(x) d v_{g}(x) \leq C_{1} \int_{M} d_{g}\left(y_{\alpha}, x\right)^{2-n}\left|u_{\alpha}(x)\right|^{2^{\star}-2} \sum_{i=1}^{p} u_{i, \alpha}(x) d v_{g}(x) .
$$

From (6-38), we also know that

$$
d_{g}\left(x_{i, \alpha}, \partial B_{x_{0}}(2 \delta)\right) \geq \delta
$$

for $\alpha$ large. In particular, we can control the boundary terms in (6-40) thanks to Proposition 5.1 and standard elliptic theory. We thus obtain that

$$
\left|u_{\alpha}\left(y_{\alpha}\right)\right|=O\left(\max \left\{\mu_{\alpha}^{(n-2) / 2} ;\left\|u_{\infty}\right\|_{\infty}\right\}\right)+O\left(\int_{M} d_{g}\left(y_{\alpha}, x\right)^{2-n}\left|u_{\alpha}(x)\right|^{2^{\star}-1} d v_{g}(x)\right) .
$$

We can now write thanks to Step 2 that

$$
\begin{aligned}
\int_{M} d_{g}\left(y_{\alpha}, x\right)^{2-n}\left|u_{\alpha}(x)\right|^{2^{\star}-1} d v_{g}(x)= & O\left(\sum_{i=1}^{\kappa} \int_{M} d_{g}\left(y_{\alpha}, x\right)^{2-n} \Phi_{i, \alpha}^{\varepsilon}(x)^{2^{\star}-1} d v_{g}(x)\right) \\
& +O\left(\max \left\{\left\|u_{\infty}\right\|_{\infty}^{2^{\star}-1} ; \mu_{\alpha}^{(1-2 \varepsilon)(n+2) / 2}\right\} \int_{M} \frac{d_{g}\left(y_{\alpha}, x\right)^{2-n}}{r_{\alpha}(x)^{(n+2) \varepsilon}} d v_{g}(x)\right) \\
& +O\left(v_{\kappa, \alpha}^{(1-2 \varepsilon)(n+2) / 2} \int_{\left\{r_{\kappa+1, \alpha}(x) \geq R_{0} v_{\kappa, \alpha}\right\}} \frac{d_{g}\left(y_{\alpha}, x\right)^{2-n}}{\left.r_{\kappa+1, \alpha}(x)^{(n+2)(1-\varepsilon)} d v_{g}(x)\right)}\right. \\
& +O\left(\int_{\left\{r_{\kappa+1, \alpha}(x) \leq R_{0} v_{\kappa, \alpha}\right\}} d_{g}\left(y_{\alpha}, x\right)^{2-n}\left|\mathcal{U}^{\alpha}\right|^{2^{\star}-1} d v_{g}(x)\right) .
\end{aligned}
$$

Since $0<\varepsilon<\frac{1}{n+2}$, it follows from Giraud's lemma that

$$
\int_{M} d_{g}\left(y_{\alpha}, x\right)^{2-n} r_{\alpha}(x)^{-(n+2) \varepsilon} d v_{g}(x)=O(1) .
$$


We can also write, for $1 \leq i \leq \kappa$,

$$
\begin{aligned}
\int_{M} d_{g}\left(y_{\alpha}, x\right)^{2-n} & \Phi_{i, \alpha}^{\varepsilon}(x)^{2^{\star}-1} d v_{g}(x) \\
= & O\left(\mu_{i, \alpha}^{-(1-2 \varepsilon)(n+2) / 2} \int_{\left\{d_{g}\left(x_{i, \alpha}, x\right) \leq \mu_{i, \alpha}\right\}} d_{g}\left(y_{\alpha}, x\right)^{2-n} d_{g}\left(x_{i, \alpha}, x\right)^{-(n+2) \varepsilon} d v_{g}(x)\right) \\
& \quad+O\left(\mu_{i, \alpha}^{(1-2 \varepsilon)(n+2) / 2} \int_{\left\{d_{g}\left(x_{i, \alpha}, x\right) \geq \mu_{i, \alpha}\right\}} d_{g}\left(y_{\alpha}, x\right)^{2-n} d_{g}\left(x_{i, \alpha}, x\right)^{-(n+2)(1-\varepsilon)} d v_{g}(x)\right)
\end{aligned}
$$

thanks to (5-8) and (6-4). Direct computations, using Giraud's lemma and the inequalities $0<\varepsilon<\frac{1}{n+2}$, lead then to

$$
\int_{M} d_{g}\left(y_{\alpha}, x\right)^{2-n} \Phi_{i, \alpha}^{\varepsilon}(x)^{2^{\star}-1} d v_{g}(x)=O\left(B_{i, \alpha}\left(y_{\alpha}\right)\right) .
$$

By direct computations, using Giraud's lemma, the inequalities $0<\varepsilon<\frac{1}{n+2}$ and (6-35), we also get

$$
\begin{aligned}
v_{\kappa, \alpha}^{(1-2 \varepsilon)(n+2) / 2} \int_{\left\{r_{\kappa+1, \alpha}(x) \geq R_{0} v_{\kappa, \alpha}\right\}} d_{g}\left(y_{\alpha}, x\right)^{2-n} r_{\kappa+1, \alpha}(x)^{-(n+2)(1-\varepsilon)} d v_{g}(x) & =O\left(v_{\kappa, \alpha}^{(n-2) / 2} R_{\kappa+1, \alpha}\left(y_{\alpha}\right)^{2-n}\right),
\end{aligned}
$$

while, using (6-35), the fact that $v_{\kappa, \alpha} \geq \mu_{\kappa+1, \alpha}$, and Hölder's inequalities, we also have

$$
\int_{\left\{r_{\kappa+1, \alpha}(x) \leq R_{0} v_{\kappa, \alpha}\right\}} d_{g}\left(y_{\alpha}, x\right)^{2-n}\left|\mathcal{U}_{\alpha}\right|^{2^{\star}-1} d v_{g}(x)=O\left(v_{\kappa, \alpha}^{(n-2) / 2} R_{\kappa+1, \alpha}\left(y_{\alpha}\right)^{2-n}\right) .
$$

Coming back to (6-42) with (6-43)-(6-47), we obtain a contradiction with the assumption that (6-32) does not hold true. This proves Step 3.

The fourth step in the proof of Proposition 6.1 is as follows. The constants $C>0$ in the statement of this step and its proof are independent of $\alpha$ and built on $C_{\kappa}$. They may change from line to line.

Step 4. There exists $C>0$ such that for any sequence $\left(y_{\alpha}\right)$ of points in $M$,

$$
\begin{aligned}
& \left|u_{\alpha}\left(y_{\alpha}\right)-u_{\infty}\left(y_{\alpha}\right)-\sum_{i=1}^{\kappa} B_{i, \alpha}\left(y_{\alpha}\right) \Lambda_{i}\right| \\
& \quad \leq \varepsilon_{\alpha}\left\|u_{\infty}\right\|_{\infty}+o\left(\sum_{i=1}^{\kappa} B_{i, \alpha}\left(y_{\alpha}\right)\right)+C\left(\mu_{\alpha}^{(n-2) / 2}+v_{\kappa, \alpha}^{(n-2) / 2} R_{\kappa+1, \alpha}\left(y_{\alpha}\right)^{2-n}\right)
\end{aligned}
$$

where $\varepsilon_{\alpha} \rightarrow 0$ as $\alpha \rightarrow+\infty$.

Proof of Step 4. Let $\left(y_{\alpha}\right)$ be a sequence of points in $M$. Assume first that

$$
R_{\kappa+1, \alpha}\left(y_{\alpha}\right)=O\left(v_{\kappa, \alpha}\right) .
$$

If $R_{\kappa+1, \alpha}\left(y_{\alpha}\right)=\mathscr{D}_{\alpha}\left(y_{\alpha}\right)$, we can apply the last equation in (4-4) of Proposition 4.2 to obtain

$$
\left|u_{\alpha}\left(y_{\alpha}\right)-u_{\infty}\left(y_{\alpha}\right)-\sum_{i=1}^{\kappa} B_{i, \alpha}\left(y_{\alpha}\right) \Lambda_{i}\right| \leq C v_{\kappa, \alpha}^{(n-2) / 2} R_{\kappa+1, \alpha}\left(y_{\alpha}\right)^{2-n} .
$$

In particular, the estimate of Step 4 holds true. If $\mathscr{D}_{\alpha}\left(y_{\alpha}\right)<R_{\kappa+1, \alpha}\left(y_{\alpha}\right)$, then from Step 1 and (6-48) we obtain the existence of some $1 \leq i \leq \kappa$ such that

$$
d_{g}\left(x_{i, \alpha}, y_{\alpha}\right)+\mu_{i, \alpha}<R_{\kappa+1, \alpha}\left(y_{\alpha}\right)=O\left(\mu_{\kappa, \alpha}\right) .
$$


This implies the following facts:

$$
\begin{gathered}
\mu_{i, \alpha}=O\left(\mu_{\kappa, \alpha}\right), \\
d_{g}\left(x_{i, \alpha}, y_{\alpha}\right)=O\left(\mu_{i, \alpha}\right), \\
R_{\kappa+1, \alpha}\left(y_{\alpha}\right) \geq \mu_{i, \alpha} .
\end{gathered}
$$

Using (4-5) in Proposition 4.2 we get

$$
\left|u_{\alpha}\left(y_{\alpha}\right)-u_{\infty}\left(y_{\alpha}\right)-\sum_{i=1}^{\kappa} B_{i, \alpha}\left(y_{\alpha}\right) \Lambda_{i}\right|=o\left(B_{i, \alpha}\left(y_{\alpha}\right)\right),
$$

and the estimate of Step 4 holds also in this case. As a consequence, we may assume below that

$$
\frac{R_{\kappa+1, \alpha}\left(y_{\alpha}\right)}{v_{\kappa, \alpha}} \rightarrow+\infty \quad \text { as } \alpha \rightarrow+\infty .
$$

The rest of the proof is based on controlling the different terms we get from Green's representation formula. We let $\mathscr{H}$ be the Green's function of the Laplacian on $M$ normalized such that $\mathscr{H}(x, y) \geq 1$ for all $x, y \in M, x \neq y$. Then (5-25) holds and moreover

$$
(x, y) \stackrel{\Phi}{\mapsto} d_{g}(x, y)^{n-2} \mathscr{H}(x, y)
$$

extends to a continuous function in $M \times M$ whose value on the diagonal is

$$
\Phi(x, x)=\frac{1}{(n-2) \omega_{n-1}}
$$

for all $x$. Now we write, for any $i \in\{1, \ldots, p\}$,

$$
u_{i, \alpha}\left(y_{\alpha}\right)-u_{i, \infty}\left(y_{\alpha}\right)=\frac{1}{V_{g}} \int_{M}\left(u_{i, \alpha}-u_{i, \infty}\right) d v_{g}+\int_{M} \mathscr{H}\left(x, y_{\alpha}\right) \Delta_{g}\left(u_{i, \alpha}-u_{i, \infty}\right)(x) d v_{g}(x) .
$$

Since $\left(\Phi_{\kappa}\right)$ holds true, we can write

$$
\left|\int_{M}\left(u_{i, \alpha}-u_{i, \infty}\right) d v_{g}\right| \leq C \mu_{\alpha}^{(n-2) / 2}+\varepsilon_{\alpha}\left\|u_{\infty}\right\|_{\infty},
$$

where $\varepsilon_{\alpha} \rightarrow 0$ as $\alpha \rightarrow+\infty$. Thus we can transform (6-50) into

$$
\left|u_{i, \alpha}\left(y_{\alpha}\right)-u_{i, \infty}\left(y_{\alpha}\right)-\int_{M} \mathscr{H}\left(x, y_{\alpha}\right) \Delta_{g}\left(u_{i, \alpha}-u_{i, \infty}\right)(x) d v_{g}(x)\right| \leq C \mu_{\alpha}^{(n-2) / 2}+\varepsilon_{\alpha}\left\|u_{\infty}\right\|_{\infty} .
$$

In view of the equations satisfied by the $\bigcup_{\alpha}$ 's and $\bigcup_{\infty}$, we can now write

$$
\begin{aligned}
\Delta_{g}\left(u_{i, \alpha}-u_{i, \infty}\right)= & \left|u_{\alpha}\right|^{2^{\star}-2} u_{i, \alpha}-\left|u_{\infty}\right|^{2^{\star}-2} u_{i, \infty}-\sum_{j=1}^{p} A_{i j}^{\alpha} u_{j, \alpha}+\sum_{j=1}^{p} A_{i j} u_{j, \infty} \\
=\left|u_{\alpha}-u_{\infty}\right|^{2^{\star}-2}\left(u_{i, \alpha}-u_{i, \infty}\right)+\left(\left|u_{\alpha}\right|^{2^{\star}-2}-\left|u_{\alpha}-u_{\infty}\right|^{2^{\star}-2}\right)\left(u_{i, \alpha}-u_{i, \infty}\right) & \\
& \quad+\left(\left|u_{\alpha}\right|^{2^{\star}-2}-\left|u_{\infty}\right|^{2^{\star}-2}\right) u_{i, \infty}-\sum_{j=1}^{p} A_{i j}^{\alpha}\left(u_{j, \alpha}-u_{j, \infty}\right)+\sum_{j=1}^{p}\left(A_{i j}-A_{i j}^{\alpha}\right) u_{j, \infty} .
\end{aligned}
$$


Thus we obtain

$$
\begin{aligned}
& \mid u_{i, \alpha}\left(y_{\alpha}\right)-u_{i, \infty}\left(y_{\alpha}\right)-\int_{M} \mathscr{H}_{y_{\alpha}}\left|u_{\alpha}-u_{\infty}\right|^{2^{\star}-2}\left(u_{i, \alpha}-u_{i, \infty}\right) d v_{g} \mid \\
& \leq\left|\int_{M} \mathscr{H}_{y_{\alpha}}\left(\left|u_{\alpha}\right|^{2^{\star}-2}-\left|u_{\alpha}-u_{\infty}\right|^{2^{\star}-2}\right)\left(u_{i, \alpha}-u_{i, \infty}\right) d v_{g}\right| \\
&+\left|\int_{M} \mathscr{H}_{y_{\alpha}}\left(\left|u_{\alpha}\right|^{2^{\star}-2}-\left|u_{\infty}\right|^{2^{\star}-2}\right) u_{i, \infty} d v_{g}\right|+\left|\sum_{j=1}^{p} \int_{M} \mathscr{H}_{y_{\alpha}}\left(A_{i j}-A_{i j}^{\alpha}\right)(x) u_{j, \infty} d v_{g}\right| \\
&+\left|\sum_{j=1}^{p} \int_{M} \mathscr{H}_{y_{\alpha}} A_{i j}^{\alpha}\left(u_{j, \alpha}-u_{j, \infty}\right) d v_{g}\right|+C \mu_{\alpha}^{(n-2) / 2}+\varepsilon_{\alpha}\left|u_{\infty}\right|_{\infty}
\end{aligned}
$$

where $\mathscr{H}_{y_{\alpha}}(x)=\mathscr{H}\left(y_{\alpha}, x\right)$ for all $x$. The convergence of the $A_{\alpha}$ 's to $A$, together with (5-25), implies that

$$
\sum_{j=1}^{p} \int_{M} \mathscr{H}_{y_{\alpha}}\left(A_{i j}-A_{i j}^{\alpha}\right) u_{j, \infty} d v_{g}=\varepsilon_{\alpha}\left\|u_{\infty}\right\|_{\infty}
$$

where $\varepsilon_{\alpha} \rightarrow 0$ as $\alpha \rightarrow+\infty$. Now we get with (5-25) that

$$
\left|\sum_{j=1}^{p} \int_{M} \mathscr{H}_{y_{\alpha}} A_{i j}^{\alpha}\left(u_{j, \alpha}-u_{j, \infty}\right) d v_{g}\right| \leq p C\left\|A_{\alpha}\right\|_{\infty} \int_{M} d_{g}\left(y_{\alpha}, x\right)^{2-n}\left|\mathcal{U}_{\alpha}(x)-\boldsymbol{u}_{\infty}(x)\right| d v_{g}(x) .
$$

Thanks to $\left(\Phi_{\kappa}\right)$, we can write

$$
\left|u_{\alpha}(x)-u_{\infty}(x)\right| \leq D_{1}\left(\sum_{j=1}^{\kappa} B_{j, \alpha}(x)+\mu_{\kappa, \alpha}^{(n-2) / 2} R_{\kappa+1, \alpha}(x)^{2-n}+\mu_{\alpha}^{(n-2) / 2}\right)+\varepsilon_{\alpha}\left\|u_{\infty}\right\|_{\infty}
$$

for some $D_{1}>0$, where $\varepsilon_{\alpha} \rightarrow 0$ as $\alpha \rightarrow+\infty$, while, thanks to Step 3, we have

$$
\left|u_{\alpha}(x)-u_{\infty}(x)\right| \leq D_{2}\left(\sum_{j=1}^{\kappa} B_{j, \alpha}(x)+v_{\kappa, \alpha}^{(n-2) / 2} R_{\kappa+1, \alpha}(x)^{2-n}+\left\|u_{\infty}\right\|_{\infty}\right)
$$

for some $D_{2}>0$. Thus we can write

$$
\begin{aligned}
\left|\sum_{j=1}^{p} \int_{M} \mathcal{H}_{y_{\alpha}} A_{i j}^{\alpha}\left(u_{j, \alpha}-u_{j, \infty}\right) d v_{g}\right| \leq C & \left.\sum_{j=1}^{\kappa} \int_{M} d_{g}\left(y_{\alpha}, x\right)^{2-n} B_{j, \alpha}(x) d v_{g}(x)\right)+\varepsilon_{\alpha}\left\|u_{\infty}\right\|_{\infty}+C \mu_{\alpha}^{(n-2) / 2} \\
& +C \mu_{\kappa, \alpha}^{(n-2) / 2} \int_{\left\{R_{\kappa+1, \alpha}(x) \geq \eta_{\alpha}\right\}} d_{g}\left(y_{\alpha}, x\right)^{2-n} R_{\kappa+1, \alpha}(x)^{2-n} d v_{g}(x) \\
& +C v_{\kappa, \alpha}^{(n-2) / 2} \int_{\left\{R_{\kappa+1, \alpha}(x) \leq \eta_{\alpha}\right\}} d_{g}\left(y_{\alpha}, x\right)^{2-n} R_{\kappa+1, \alpha}(x)^{2-n} d v_{g}(x),
\end{aligned}
$$

where $\eta_{\alpha}=2 \operatorname{diam}_{g} M$ if $U_{\infty} \equiv 0, \eta_{\alpha}=\mu_{\kappa, \alpha}^{1 / 2}$ otherwise, and $\operatorname{diam}_{g} M$ is the diameter of $M$ with respect to $g$. Simple computations, using Giraud's lemma, then lead to the estimate

$$
\begin{aligned}
\mid \sum_{j=1}^{p} \int_{M} \mathscr{H}_{y_{\alpha}} A_{i j}^{\alpha}\left(u_{j, \alpha}\right. & \left.-u_{j, \infty}\right) d v_{g} \mid \\
& \leq o\left(\sum_{j=1}^{\kappa}\left|B_{j}^{\alpha}\left(y_{\alpha}\right)\right|\right)+C \mu_{\alpha}^{(n-2) / 2}+\varepsilon_{\alpha}\left\|u_{\infty}\right\|_{\infty}+C v_{\kappa, \alpha}^{(n-2) / 2} R_{\kappa+1, \alpha}\left(y_{\alpha}\right)^{2-n} .
\end{aligned}
$$


If $u_{\infty} \equiv 0$, we have

$$
\int_{M} \mathcal{H}_{y_{\alpha}}\left(\left|u_{\alpha}\right|^{2^{\star}-2}-\left|u_{\infty}\right|^{2^{\star}-2}\right) u_{i, \infty} d v_{g}=0,
$$

while, if $u_{\infty} \not \equiv 0$, we write, thanks to Proposition 5.1,

$$
\begin{aligned}
\int_{M} \mathscr{H}_{y_{\alpha}}\left(\left|u_{\alpha}\right|^{2^{\star}-2}-\left|u_{\infty}\right|^{2^{\star}-2}\right) u_{i, \infty} d v_{g} & =o(1)+\int_{\left\{\mathscr{D}_{\alpha} \leq \mu_{\alpha}^{1 / 4}\right\}} \mathcal{H}_{y_{\alpha}}\left(\left|u_{\alpha}\right|^{2^{\star}-2}-\left|u_{\infty}\right|^{2^{\star}-2}\right) u_{i, \infty} d v_{g} \\
& =o(1)+O\left(\int_{\left\{\mathscr{D}_{\alpha} \leq \mu_{\alpha}^{1 / 4}\right\}} d_{g}\left(x, y_{\alpha}\right)^{2-n}\left|u_{\alpha}(x)\right|^{2^{\star}-2} d v_{g}(x)\right) .
\end{aligned}
$$

Now we use Step 3 and we briefly distinguish the $n=3,4,5$, and $n \geq 6$ cases in the forthcoming computations. We let $\left(R_{\alpha}\right)_{\alpha}$ be suitably chosen such that $R_{\alpha} \rightarrow+\infty$ as $\alpha \rightarrow+\infty$. Assuming that $n=3,4,5$, we write with (6-55) that

$$
\begin{aligned}
\int_{M} \mathcal{H}_{y_{\alpha}}\left(\left|u_{\alpha}\right|^{2^{\star}-2}-\left|u_{\infty}\right|^{2^{\star}-2}\right) u_{i, \infty} d v_{g} & \\
=o(1) & +O\left(\int_{\left\{R_{\kappa+1, \alpha} \leq R_{\kappa+1, \alpha}\left(y_{\alpha}\right) / R_{\alpha}\right\}} d_{g}\left(x, y_{\alpha}\right)^{2-n}\left|u_{\alpha}(x)\right|^{2^{\star}-2} d v_{g}(x)\right) \\
& +O\left(\int_{\left\{R_{\kappa+1, \alpha} \geq R_{\kappa+1, \alpha}\left(y_{\alpha}\right) / R_{\alpha}\right\} \cap\left\{\mathfrak{D}_{\alpha} \leq \mu_{\alpha}^{1 / 4}\right\}} d_{g}\left(x, y_{\alpha}\right)^{2-n}\left|u_{\alpha}(x)\right|^{2^{\star}-2} d v_{g}(x)\right) \\
=o(1) & +O\left(\sum_{j=1}^{\kappa} \int_{M} d_{g}\left(x, y_{\alpha}\right)^{2-n} B_{j, \alpha}(x)^{2^{\star}-2} d v_{g}(x)\right) \\
& +O\left(v_{\kappa, \alpha}^{2} \int_{\left\{R_{\kappa+1, \alpha} \geq R_{\kappa+1, \alpha}\left(y_{\alpha}\right) / R_{\alpha}\right\}} d_{g}\left(x, y_{\alpha}\right)^{2-n} R_{\kappa+1, \alpha}(x)^{-4} d v_{g}(x)\right) \\
=o(1) & +o\left(\sum_{j=1}^{\kappa} B_{j, \alpha}\left(y_{\alpha}\right)\right)+o\left(v_{\kappa, \alpha}^{(n-2) / 2} R_{\kappa+1, \alpha}\left(y_{\alpha}\right)^{2-n}\right),
\end{aligned}
$$

and, assuming that $n \geq 6$, since $2^{\star}-2 \in(0,1]$ in this case, we get from $(6-55)$ that

$$
\begin{aligned}
& \int_{M} \mathcal{H}_{y_{\alpha}}\left(\left|u_{\alpha}\right|^{2^{\star}-2}-\left|u_{\infty}\right|^{2^{\star}-2}\right) u_{i, \infty} d v_{g}=o(1)+ o\left(\int_{\left\{\mathscr{D}_{\alpha} \leq \mu_{\alpha}^{1 / 4}\right\}} d_{g}\left(x, y_{\alpha}\right)^{2-n}\left|u_{\alpha}(x)\right| d v_{g}(x)\right) \\
&=o(1)+ o\left(\sum_{j=1}^{\kappa} \int_{M} d_{g}\left(x, y_{\alpha}\right)^{2-n} B_{j, \alpha}(x) d v_{g}(x)\right) \\
&+O\left(v_{\kappa, \alpha}^{(n-2) / 2} \int_{M} d_{g}\left(x, y_{\alpha}\right)^{2-n} R_{\kappa+1, \alpha}(x)^{2-n} d v_{g}(x)\right) \\
&=o(1)+o\left(\sum_{j=1}^{\kappa} B_{j, \alpha}\left(y_{\alpha}\right)\right)+o\left(v_{\kappa, \alpha}^{(n-2) / 2} R_{\kappa+1, \alpha}\left(y_{\alpha}\right)^{2-n}\right)
\end{aligned}
$$


Thus, in all cases,

$$
\begin{aligned}
\int_{M} \mathscr{H}_{y_{\alpha}}\left(\left|\boldsymbol{U}_{\alpha}\right|^{2^{\star}-2}-\left|\mathcal{U}_{\infty}\right|^{2^{\star}-2}\right) & u_{i, \infty} d v_{g} \\
& =o\left(\sum_{j=1}^{\kappa} B_{j, \alpha}\left(y_{\alpha}\right)\right)+o\left(v_{\kappa, \alpha}^{(n-2) / 2} R_{\kappa+1, \alpha}\left(y_{\alpha}\right)^{2-n}\right)+\varepsilon_{\alpha}\left\|u_{\infty}\right\|_{\infty} .
\end{aligned}
$$

Similarly, if $u_{\infty} \equiv 0$, then

$$
\int_{M} \mathscr{H}_{y_{\alpha}}\left(\left|u_{\alpha}\right|^{2^{\star}-2}-\left|u_{\alpha}-u_{\infty}\right|^{2^{\star}-2}\right)\left(u_{i, \alpha}-u_{i, \infty}\right) d v_{g}=0
$$

while, if $u^{\infty} \not \equiv 0$, we can write

$$
\begin{aligned}
\int_{M} \mathscr{H}_{y_{\alpha}}\left(\left|u_{\alpha}\right|^{2^{\star}-2}-\left|u_{\alpha}-u_{\infty}\right|^{2^{\star}-2}\right)\left(u_{i, \alpha}-\right. & \left.u_{i, \infty}\right) d v_{g} \\
& =o(1)+o\left(\int_{\left\{R_{\alpha}(x) \leq \mu_{\alpha}^{1 / 4}\right\}} d_{g}\left(y_{\alpha}, x\right)^{2-n}\left|u_{\alpha}(x)\right|^{2^{\star}-2} d v_{g}(x)\right) \\
& =o(1)+o\left(\sum_{j=1}^{\kappa} B_{j, \alpha}\left(y_{\alpha}\right)\right)+o\left(v_{\kappa, \alpha}^{(n-2) / 2} R_{\kappa+1, \alpha}\left(y_{\alpha}\right)^{2-n}\right) .
\end{aligned}
$$

Thus we have obtained

$$
\begin{aligned}
\int_{M} \mathscr{H}_{y_{\alpha}}\left(\left|u_{\alpha}\right|^{2^{\star}-2}-\left|u_{\alpha}-u_{\infty}\right|^{2^{\star}-2}\right)\left(u_{i, \alpha}-u_{i, \infty}\right) d v_{g} \\
=o\left(\sum_{j=1}^{\kappa} B_{j, \alpha}\left(y_{\alpha}\right)\right)+o\left(v_{\kappa, \alpha}^{(n-2) / 2} R_{\kappa+1, \alpha}\left(y_{\alpha}\right)^{2-n}\right)+\varepsilon_{\alpha}\left\|u_{\infty}\right\|_{\infty} .
\end{aligned}
$$

Coming back to (6-52): thanks to (6-53)-(6-57), we now obtain

$$
\begin{aligned}
\mid \boldsymbol{u}_{\alpha}\left(y_{\alpha}\right)-\boldsymbol{u}_{\infty}\left(y_{\alpha}\right) & -\int_{M} \mathscr{H}_{y_{\alpha}}\left|\boldsymbol{u}_{\alpha}-\boldsymbol{u}_{\infty}\right|^{2^{\star}-2}\left(u_{\alpha}-\boldsymbol{u}_{\infty}\right) d v_{g} \mid \\
& \leq o\left(\sum_{j=1}^{\kappa} B_{j, \alpha}\left(y_{\alpha}\right)\right)+o\left(v_{\kappa, \alpha}^{(n-2) / 2} R_{\kappa+1, \alpha}\left(y_{\alpha}\right)^{2-n}\right)+\varepsilon_{\alpha}\left\|u_{\infty}\right\|_{\infty}+C \mu_{\alpha}^{(n-2) / 2} .
\end{aligned}
$$

Using (4-5), (5-25), and the extension property of $d_{g}(x, y)^{n-2} \mathscr{H}(x, y)$ mentioned above, we can find a sequence $\left(R_{\alpha}\right)_{\alpha}$ such that $R_{\alpha} \rightarrow+\infty$ as $\alpha \rightarrow+\infty$, that

$$
\left|\int_{M_{i, \alpha}} \mathscr{H}_{y_{\alpha}}\right| u_{\alpha}-\left.u_{\infty}\right|^{2^{\star}-2}\left(u_{\alpha}-u_{\infty}\right) d v_{g}-B_{i, \alpha}\left(y_{\alpha}\right) \Lambda_{i} \mid \leq C \mu_{\alpha}^{(n-2) / 2}+o\left(B_{i, \alpha}\left(y_{\alpha}\right)\right)
$$

for all $i \in\{1, \ldots, \kappa\}$, and that the sets

$$
M_{i, \alpha}=B_{x_{i, \alpha}}\left(R_{\alpha} \mu_{i, \alpha}\right) \backslash \bigcup_{i+1 \leq j \leq N} B_{x_{j, \alpha}}\left(R_{\alpha}^{-1} \mu_{i, \alpha}\right)
$$

are disjoint. 
Then we can write thanks to Proposition 5.1, Step 3, and (6-49), that

$$
\begin{aligned}
& \int_{M \backslash \bigcup_{1 \leq i \leq k} M_{i, \alpha}} d_{g}\left(y_{\alpha}, x\right)^{2-n}\left|U_{\alpha}(x)-u_{\infty}(x)\right|^{2^{\star}-1} d v_{g}(x) \\
& \leq \varepsilon_{\alpha}\left\|u_{\infty}\right\|_{\infty}+C \mu_{\alpha}^{(n-2) / 2}+C \sum_{j=1}^{\kappa} \int_{M_{\alpha}} d_{g}\left(y_{\alpha}, x\right)^{2-n} B_{j, \alpha}(x)^{2^{\star}-1} d v_{g}(x) \\
& \quad+C v_{\kappa, \alpha}^{(n+2) / 2} \int_{\left\{R_{\kappa+1, \alpha} \geq v_{\kappa, \alpha}\right\}} \frac{d_{g}\left(y_{\alpha}, x\right)^{2-n}}{R_{\kappa+1, \alpha}(x)^{(n+2)}} d v_{g}(x)+C v_{\kappa, \alpha}^{(n-2) / 2} R_{\kappa+1, \alpha}\left(y_{\alpha}\right)^{2-n} \\
& \leq \varepsilon_{\alpha}\left\|u_{\infty}\right\|_{\infty}+C \mu_{\alpha}^{(n-2) / 2}+o\left(\sum_{j=1}^{\kappa} B_{j, \alpha}\left(y_{\alpha}\right)\right)+C v_{\kappa, \alpha}^{(n-2) / 2} R_{\kappa+1, \alpha}\left(y_{\alpha}\right)^{2-n}
\end{aligned}
$$

Coming back to (6-58), this ends the proof of Step 4.

Step 5. $v_{\kappa, \alpha}=\mu_{\kappa+1, \alpha}$.

Proof of Step 5. We proceed by contradiction and thus assume that there exists $i \in\{1, \ldots, \kappa\}$ and a sequence $\left(y_{\alpha}\right)$ of points in $\tilde{\Omega}_{i, \alpha}^{\varepsilon}$ such that

$$
v_{\kappa, \alpha}^{(1-2 \varepsilon)(n-2) / 2} \Psi_{\alpha}^{\varepsilon}\left(y_{\alpha}\right)=\Phi_{i, \alpha}^{\varepsilon}\left(y_{\alpha}\right) .
$$

Since $y_{\alpha} \in \tilde{\Omega}_{i, \alpha}^{\varepsilon}$, we know that

$$
\Phi_{i, \alpha}^{\varepsilon}\left(y_{\alpha}\right) \geq \Phi_{j, \alpha}^{\varepsilon}\left(y_{\alpha}\right)
$$

for all $1 \leq j \leq \kappa$ and that

$$
d_{g}\left(x_{i, \alpha}, y_{\alpha}\right)^{2}\left|u_{\alpha}\left(y_{\alpha}\right)-u_{\infty}\left(y_{\alpha}\right)-\sum_{j=1}^{\kappa} B_{j, \alpha}\left(y_{\alpha}\right) \Lambda_{j}\right|^{2^{\star}-2} \geq D(\varepsilon)
$$

Clearly,

$$
d_{g}\left(x_{i, \alpha}, x\right)^{2} B_{i, \alpha}(x)^{2^{\star}-2}=O(1) .
$$

We now claim that

$$
d_{g}\left(x_{i, \alpha}, y_{\alpha}\right)^{2} B_{j, \alpha}\left(y_{\alpha}\right)^{2^{\star}-2} \rightarrow 0 \quad \text { as } \alpha \rightarrow+\infty
$$

for all $1 \leq j \leq \kappa, j \neq i$. In order to prove (6-63), we proceed by contradiction once again and assume that there exists $1 \leq j \leq \kappa, j \neq i$, such that

$$
\left(d_{g}\left(x_{j, \alpha}, y_{\alpha}\right)+\mu_{j, \alpha}\right)^{2}=O\left(\mu_{j, \alpha} d_{g}\left(x_{i, \alpha}, y_{\alpha}\right)\right) .
$$

Since $\Phi_{i, \alpha}^{\varepsilon}\left(y_{\alpha}\right) \geq \Phi_{j, \alpha}^{\varepsilon}\left(y_{\alpha}\right)$, we then get

$$
\left(d_{g}\left(x_{i, \alpha}, y_{\alpha}\right)+\mu_{i, \alpha}\right)^{1-\varepsilon}=O\left(\mu_{j, \alpha}^{\varepsilon} \mu_{i, \alpha}^{1-2 \varepsilon}\right),
$$

so $\mu_{i, \alpha}=O\left(\mu_{j, \alpha}\right)$ and $d_{g}\left(x_{i, \alpha}, y_{\alpha}\right)^{1-\varepsilon}=O\left(\mu_{j, \alpha}^{\varepsilon} \mu_{i, \alpha}^{1-2 \varepsilon}\right)$. Coming back to (6-64), we also obtain $\mu_{j, \alpha}=$ $O\left(\mu_{i, \alpha}\right)$ and $d_{g}\left(x_{j, \alpha}, y_{\alpha}\right)=O\left(\mu_{i, \alpha}\right)$. This contradicts the first equation in (4-5). Thus (6-63) is proved.

Applying Step 4, we get from (6-61), (6-62), and (6-63) that

$$
R_{\kappa+1, \alpha}\left(y_{\alpha}\right)^{2}=O\left(v_{\kappa, \alpha} d_{g}\left(x_{i, \alpha}, y_{\alpha}\right)\right) \text {. }
$$


Using (5-8) and (6-59), we also have

$$
\left(d_{g}\left(x_{i, \alpha}, y_{\alpha}\right)+\mu_{i, \alpha}\right)^{2(1-\varepsilon)}=O\left(\mu_{i, \alpha}^{1-2 \varepsilon} v_{\kappa, \alpha}^{2 \varepsilon-1} R_{\kappa+1, \alpha}\left(y_{\alpha}\right)^{2(1-\varepsilon)}\right),
$$

so that, with (6-65) and Step 1, we get that $\mu_{i, \alpha}=O\left(\mu_{\kappa, \alpha}\right)$, that $d_{g}\left(x_{i, \alpha}, y_{\alpha}\right)=O\left(\mu_{i, \alpha}\right)$ and that $\mu_{i, \alpha}=$ $O\left(R_{\kappa+1, \alpha}\left(y_{\alpha}\right)\right)$. Using the second equation in (4-5) of Proposition 4.2 we then obtain

$$
d_{g}\left(x_{i, \alpha}, y_{\alpha}\right)^{2}\left|\mathcal{U}_{\alpha}\left(y_{\alpha}\right)-B_{i, \alpha} \Lambda_{i}\left(y_{\alpha}\right)\right|^{2^{\star}-2} \rightarrow 0 \quad \text { as } \alpha \rightarrow+\infty .
$$

This contradicts (6-61) thanks to (6-63). Step 5 is proved.

Conclusion of the proof of Proposition 6.1. By Proposition 5.1 we know that $\left(\mathscr{I}_{1}\right)$ holds true. By Steps 4 and 5, and by induction, it follows that $\left(\mathscr{I}_{N}\right)$ holds true. It remains to prove that $\left(\mathscr{I}_{N+1}\right)$ also holds true. For this we proceed with similar arguments to those developed in the proof of Step 4. We let $\left(y_{\alpha}\right)$ be a sequence of points in $M$ and write, for any $i=1, \ldots, p$,

$$
u_{i, \alpha}\left(y_{\alpha}\right)-u_{i, \infty}\left(y_{\alpha}\right)=\frac{1}{V_{g}} \int_{M}\left(u_{i, \alpha}-u_{i, \infty}\right) d v_{g}+\int_{M} \mathscr{H}_{y_{\alpha}} \Delta_{g}\left(u_{i, \alpha}-u_{i, \infty}\right) d v_{g},
$$

where $\mathscr{H}_{y_{\alpha}}(\cdot)=\mathscr{H}\left(\cdot, y_{\alpha}\right)$ and $\mathscr{H}$ is the Green's function of $\Delta_{g}$ normalized so that $\mathscr{H} \geq 1$. Since $\left(\mathscr{I}_{N}\right)$ holds true,

$$
\int_{M}\left|u_{i, \alpha}-u_{i, \infty}\right| d v_{g} \leq C \mu_{\alpha}^{(n-2) / 2}+\varepsilon_{\alpha}\left\|u_{\infty}\right\|_{\infty},
$$

where $C>0$ is independent of $\alpha$, and $\varepsilon_{\alpha} \rightarrow 0$ as $\alpha \rightarrow+\infty$. Using the equations satisfied by the $u_{\alpha}$ 's and $u_{\infty}$, but also $\left(\mathscr{I}_{N}\right)$, mimicking what was done in the proof of Step 4, we get with (6-66) that

$$
\begin{aligned}
& \left|u_{\alpha}\left(y_{\alpha}\right)-u_{\infty}\left(y_{\alpha}\right)-\int_{M} \mathscr{H}_{y_{\alpha}}\right| u_{\alpha}-\left.u_{\infty}\right|^{2^{\star}-2}\left(u_{i, \alpha}-u_{i, \infty}\right) d v_{g} \mid \\
& \leq C \mu_{\alpha}^{(n-2) / 2}+o\left(\sum_{i=1}^{N} B_{i, \alpha}\left(y_{\alpha}\right)\right)+\varepsilon_{\alpha}\left\|u_{\infty}\right\|_{\infty} .
\end{aligned}
$$

We also have

$$
\begin{aligned}
\left|\int_{M} \mathscr{H}_{y_{\alpha}}\right| u_{\alpha}-\left.u_{\infty}\right|^{2^{\star}-2}\left(u_{i, \alpha}-u_{i, \infty}\right) d v_{g}-\sum_{i=1}^{N} & B_{i, \alpha}\left(y_{\alpha}\right) \Lambda_{i} \mid \\
& \leq C \mu_{\alpha}^{(n-2) / 2}+o\left(\sum_{i=1}^{N} B_{i, \alpha}\left(y_{\alpha}\right)\right)+\varepsilon_{\alpha}\left\|u_{\infty}\right\|_{\infty},
\end{aligned}
$$

where $C>0$ in (6-67), (6-68) is independent of $\alpha$, and $\varepsilon_{\alpha} \rightarrow 0$ as $\alpha \rightarrow+\infty$. Combining (6-67) and (6-68), we get $\left(\Phi_{N+1}\right)$. This ends the proof of Proposition 6.1.

\section{A Pohozaev identity for systems}

Let $(M, g)$ be a smooth compact Riemannian manifold. Let also $X$ be a smooth 1 -form over $M$ and $\mathcal{U}: M \rightarrow \mathbb{R}^{p}$ be a $C^{2}$-map. We define $X(\nabla \mathcal{u})$ by $X(\nabla \cup)=(\nabla \cup, X)$. This is a $p$-map with components $X(\nabla \cup)_{i}=\left(\nabla u_{i}, X\right)$ where the $u_{i}$ 's are the components of $U$. We define also $|\nabla \cup|$ and $\left(T_{\nabla} X\right)_{u}$ by $|\nabla u|^{2}=\sum_{i=1}^{p}\left|\nabla u_{i}\right|^{2}$ and $\left(T_{\nabla} X\right) u_{u}=\sum_{i=1}^{p} S_{X}^{\sharp}\left(\nabla u_{i}, \nabla u_{i}\right)$, where $S_{X}^{\sharp}$ is the $(0,2)$-tensor field we obtain 
from the $(2,0)$-tensor field $S_{X}$ by the musical isomorphism, and

$$
S_{X}=\nabla X-\frac{1}{n}\left(\operatorname{div}_{g} X\right) g
$$

For $\Omega$ a smooth bounded domain in $M$ we let $v$ be the unit outer normal to $\partial \Omega$. The Pohozaev-type identity for systems we prove is stated as follows.

Proposition 7.1. Let $(M, g)$ be a smooth compact Riemannian n-manifold, $\Omega$ be a smooth bounded domain in $M$, and $A: M \rightarrow M_{p}^{s}(\mathbb{R})$ be a $C^{1}$-map. Let $X$ be a smooth 1-form over $M$ and $U$ be a solution of (0-1). Then

$$
\begin{aligned}
& \int_{\Omega}\langle A \cup, X(\nabla \cup)\rangle_{\mathbb{R}^{p}} d v_{g}+\frac{n-2}{4 n} \int_{\Omega}\left(\Delta_{g}\left(\operatorname{div}_{g} X\right)\right)|u|^{2} d v_{g}+\frac{n-2}{2 n} \int_{\Omega}\left(\operatorname{div}_{g} X\right)\langle A \cup, \mathcal{u}\rangle_{\mathbb{R}^{p}} d v_{g} \\
& =-\int_{\Omega}\left(T_{\nabla} X\right) u_{u} d v_{g}+\frac{n-2}{2 n} \int_{\partial \Omega} X(v)|u|^{2^{\star}} d \sigma_{g}-\frac{n-2}{4 n} \int_{\partial \Omega} \partial_{\nu}\left(\operatorname{div}_{g} X\right)|u|^{2} d \sigma_{g} \\
& +\frac{n-2}{2 n} \int_{\partial \Omega}\left(\operatorname{div}_{g} X\right)\left\langle\partial_{\nu} u, u\right\rangle_{\mathbb{R}^{p}} d \sigma_{g}-\int_{\partial \Omega} B_{\partial \Omega}(u) d \sigma_{g},
\end{aligned}
$$

where $X(\nabla \cup)$ and $\left(T_{\nabla} X\right)_{u}$ are as above, $B_{\partial \Omega}(u)=\frac{1}{2} X(v)|\nabla \cup|^{2}-\left\langle X(\nabla \cup), \partial_{\nu} u\right\rangle_{\mathbb{R}^{p}}$ on $\partial \Omega$, and $\langle\cdot, \cdot\rangle_{\mathbb{R}^{p}}$ is the scalar product in $\mathbb{R}^{p}$.

Proof. Integrating by parts we easily see that for $u: M \rightarrow \mathbb{R}$ of class $C^{2}$,

$$
\int_{\Omega}(\nabla u, X) \Delta_{g} u d v_{g}=\int_{\Omega} S_{X, 2}^{\sharp}(\nabla u, \nabla u) d v_{g}+\int_{\partial \Omega}\left(\frac{1}{2} \int_{\Omega} X(v)|\nabla u|^{2}-(\nabla u, X) \partial_{\nu} u\right) d \sigma_{g},
$$

where $S_{X, 2}=\nabla X-\frac{1}{2}\left(\operatorname{div}_{g} X\right) g$. If we assume now that $U$ is a $p$-map, applying (7-3) to the components $u_{i}$ of $U$, and summing over $i$, we obtain

$$
\int_{\Omega}\left\langle X(\nabla \cup), \Delta_{g} u\right\rangle_{\mathbb{R}^{p}} d v_{g}=\int_{\Omega} S_{X, 2}^{\sharp}(\nabla \cup, \nabla \cup) d v_{g}+\int_{\partial \Omega} B_{\partial \Omega}(\mathcal{u}) d \sigma_{g} .
$$

We assume now that $\mathcal{u}$ solves (0-1) and we use the equations satisfied by $\mathcal{u}$ to explicit the left-hand side in the preceding equation. We can write

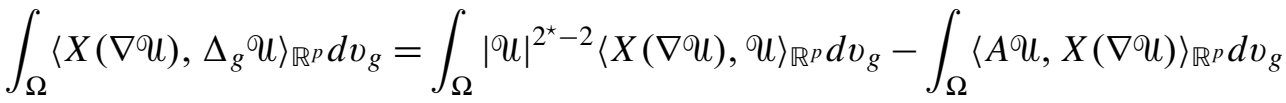

$$
\begin{aligned}
& =\frac{1}{2^{\star}} \int_{\Omega}\left(\nabla|u|^{2^{\star}}, X\right) d v_{g}-\int_{\Omega}\langle A \cup, X(\nabla \mathcal{U})\rangle_{\mathbb{R}^{p}} d v_{g} \\
& =-\frac{1}{2^{\star}} \int_{\Omega}\left(\operatorname{div}_{g} X\right)|u|^{2^{\star}} d v_{g}-\int_{\Omega}\langle A \cup, X(\nabla \cup)\rangle_{\mathbb{R}^{p}} d v_{g}+\frac{1}{2^{\star}} \int_{\partial \Omega} X(v)|u|^{2^{\star}} d \sigma_{g} .
\end{aligned}
$$

Then we get

$$
\begin{aligned}
& \int_{\Omega}\langle A \cup, X(\nabla \cup)\rangle_{\mathbb{R}^{p}} d v_{g}+\frac{1}{2^{\star}} \int_{\Omega}\left(\operatorname{div}_{g} X\right)|u|^{2^{\star}} d v_{g}+\int_{\Omega} S_{X, 2}^{\sharp}(\nabla \cup, \nabla \cup) d v_{g} \\
& =\frac{1}{2^{\star}} \int_{\partial \Omega} X(v)|u|^{2^{\star}} d \sigma_{g}-\int_{\partial \Omega} B_{\partial \Omega}(U) d \sigma_{g} .
\end{aligned}
$$


Using once again (0-1), we obtain

$$
\begin{aligned}
& \int_{\Omega} S_{X, 2}^{\sharp}(\nabla \cup, \nabla \cup) d v_{g}=\int_{\Omega} S_{X}^{\sharp}(\nabla \cup, \nabla \cup) d v_{g}-\frac{1}{2^{\star}} \int_{\Omega}\left(\operatorname{div}_{g} X\right)|\nabla \cup|^{2} d v_{g} \\
& =\int_{\Omega} S_{X}^{\sharp}(\nabla \cup, \nabla u) d v_{g}-\frac{1}{2^{\star}} \int_{\partial \Omega}\left(\operatorname{div}_{g} X\right)\left\langle\partial_{\nu} \cup, u\right\rangle_{\mathbb{R}^{p}} d \sigma_{g} \\
& +\frac{n-2}{4 n} \int_{\partial \Omega} \partial_{\nu}\left(\operatorname{div}_{g} X\right)|u|^{2} d \sigma_{g}+\frac{n-2}{4 n} \int_{\Omega}\left(\Delta_{g}\left(\operatorname{div}_{g} X\right)\right)|u|^{2} d v_{g} \\
& -\frac{1}{2^{\star}} \int_{\Omega}\left(\operatorname{div}_{g} X\right)|u|^{2^{\star}} d v_{g}+\frac{1}{2^{\star}} \int_{\Omega}\left(\operatorname{div}_{g} X\right)\langle A \cup, \mathcal{U}\rangle_{\mathbb{R}^{p}} d v_{g},
\end{aligned}
$$

and (7-2) easily follows. This ends the proof of the proposition.

The Pohozaev-type identity (7-2) is used repeatedly, with different choices of $X$, in the next section.

\section{The range of influence of blow-up points}

We start with notations and the definition of the range of influence of blow-up points. The blow-up points $x_{i, \alpha}$ of Proposition 4.2 come with vector bubbles $\left(\mathscr{B}_{\alpha}^{i}\right)_{\alpha}$ as in the same proposition. We let $\Lambda_{i}$ be the $S^{p-1}$ projection of $\left(\mathscr{B}_{\alpha}^{i}\right)_{\alpha}$, and $B_{i, \alpha}=\left|\mathscr{B}_{\alpha}^{i}\right|$ for all $i$ and all $\alpha$. As above, $\left(A_{\alpha}\right)_{\alpha}$ is a sequence of $C^{1}$ maps from $M$ to $M_{p}^{s}(\mathbb{R})$ such that $A_{\alpha} \rightarrow A$ in $C^{1}(M)$ as $\alpha \rightarrow+\infty$ for some $C^{1}$ map $A$ from $M$ to $M_{p}^{s}(\mathbb{R})$ satisfying $(\mathrm{H})$, and we order the blow-up points in such a way that

$$
\mu_{\alpha}=\mu_{1, \alpha} \geq \cdots \geq \mu_{N, \alpha},
$$

where the $\mu_{i, \alpha}$ 's are the weights of the vector bubble $\left(\mathscr{B}_{\alpha}^{i}\right)_{\alpha}$. Given $i, j \in\{1, \ldots, N\}, i \neq j$, we let $s_{i, j, \alpha}$ be given by

$$
s_{i, j, \alpha}^{2}=\frac{\mu_{i, \alpha}}{\mu_{j, \alpha}} \frac{d_{g}\left(x_{i, \alpha}, x_{j, \alpha}\right)^{2}}{n(n-2)}+\mu_{i, \alpha} \mu_{j, \alpha}=\mu_{i, \alpha} B_{j, \alpha}\left(x_{i, \alpha}\right)^{-2 /(n-2)}
$$

and we define the range of influence of the blow-up point $x_{i, \alpha}$ by

$$
r_{i, \alpha}= \begin{cases}\min _{j \in \mathscr{A}_{i}} s_{i, j, \alpha} & \text { if } u_{\infty} \equiv 0 \\ \min \left\{\min _{j \in \mathscr{A}_{i}} s_{i, j, \alpha} ; \sqrt{\mu_{i, \alpha}}\right\} & \text { if } u_{\infty} \neq \equiv .\end{cases}
$$

where

$$
\mathscr{A}_{i}=\left\{j \in\{1, \ldots, N\}, j \neq i \text { s.t. } \mu_{i, \alpha}=O\left(\mu_{j, \alpha}\right)\right\} .
$$

If $\mathscr{A}_{i}=\varnothing$ (so that, in particular, $i=1$ ) and $U_{\infty} \equiv 0$, we let by definition $r_{i, \alpha}=\frac{1}{2} i_{g}$, where $i_{g}$ is the injectivity radius of $(M, g)$. Using the first equation in (4-5) it is easily checked that

$$
\frac{s_{i, j, \alpha}}{\mu_{i, \alpha}} \rightarrow+\infty \text { as } \alpha \rightarrow+\infty \text { for all } i, j \in\{1, \ldots, N\} \text { and all } j \in \mathscr{A}_{i} .
$$

This implies in particular that

$$
\frac{r_{i, \alpha}}{\mu_{i, \alpha}} \rightarrow+\infty \quad \text { as } \alpha \rightarrow+\infty .
$$

If $j \in \mathscr{A}_{i}$ and $i \in \mathscr{A}_{j}$, we let $\lambda_{i, j} \geq 0$ be given by

$$
\lambda_{i, j}=\left(\lim _{\alpha \rightarrow+\infty} \frac{\mu_{j, \alpha}}{\mu_{i, \alpha}}\right)^{(n-2) / 2} .
$$


Given $i \in\{1, \ldots, N\}$, we also let

$$
\mathscr{B}_{i}= \begin{cases}\left\{j \in\{1, \ldots, N\}, j \neq i \text { s.t. } d_{g}\left(x_{i, \alpha}, x_{j, \alpha}\right)=O\left(r_{i, \alpha}\right)\right\} & \text { if } r_{i, \alpha} \rightarrow 0, \\ \left\{j \in\{1, \ldots, N\}, j \neq i \text { s.t. } x_{j} \in B_{x_{i}}\left(\frac{1}{2} i_{g}\right)\right\} & \text { if } r_{i, \alpha} \not \supset 0 .\end{cases}
$$

and, for $j \in \mathscr{B}_{i}$,

$$
z_{i, j}=\lim _{\alpha \rightarrow+\infty} r_{i, \alpha}^{-1} \exp _{x_{i, \alpha}}^{-1}\left(x_{j, \alpha}\right)
$$

Up to a subsequence, all these limits exist. We let $\delta_{i}>0$ be such that for any $i$ and any $j \in \mathscr{P}_{i}$,

$$
\left|z_{i, j}\right| \neq 0 \Rightarrow\left|z_{i, j}\right| \geq 10 \delta_{i}
$$

We also define $\mathscr{C}_{i}$ to be the subset of $\mathscr{B}_{i}$ given by

$$
\mathscr{C}_{i}=\left\{j \in \mathscr{B}_{i} \text { s.t. } z_{i, j}=0\right\} \cap \mathscr{A}_{i}^{c} .
$$

It can be proved that there exists a subset $\mathscr{D}_{i}$ of $\mathscr{C}_{i}$ and a family $\left(R_{i, j}\right)_{j \in \mathscr{D}_{i}}$ of positive real numbers such that the two following assertions hold true: for any $j, k \in \mathscr{D}_{i}, j \neq k$,

$$
\frac{d_{g}\left(x_{j, \alpha}, x_{k, \alpha}\right)}{s_{j, i, \alpha}} \rightarrow+\infty
$$

as $\alpha \rightarrow+\infty$, and for any $j \in \mathscr{C}_{i}$ there exists a unique $k \in \mathscr{D}_{i}$ such that

$$
\limsup _{\alpha \rightarrow+\infty} \frac{d_{g}\left(x_{j, \alpha}, x_{k, \alpha}\right)}{s_{k, i, \alpha}} \leq \frac{R_{i, k}}{20} \quad \text { and } \quad \limsup _{\alpha \rightarrow+\infty} \frac{s_{j, i, \alpha}}{s_{k, i, \alpha}} \leq \frac{R_{i, k}}{20} \text {. }
$$

We also introduce the subsets

$$
\Omega_{i, \alpha}=B_{x_{i, \alpha}}\left(\delta_{i} r_{i, \alpha}\right) \backslash \bigcup_{j \in \mathscr{D}_{i}} \Omega_{i, j, \alpha}
$$

of $M$, where

$$
\Omega_{i, j, \alpha}=B_{x_{j, \alpha}}\left(R_{i, j} s_{j, i, \alpha}\right)
$$

for all $j \in \mathscr{D}_{i}$. The $\Omega_{i, j, \alpha}$ 's are disjoint for $\alpha$ sufficiently large.

We now prove two lemmas to be used in the proof of Theorem 0.1 .

Lemma 8.1. Let $i \in\{1, \ldots, N\}$. Up to passing to a subsequence,

$$
\left|u_{\alpha}-\Lambda_{i} B_{i, \alpha}\right|=o\left(B_{i, \alpha}\right)+O\left(\mu_{i, \alpha}^{(n-2) / 2} r_{i, \alpha}^{2-n}\right)+O\left(\sum_{j \in \mathscr{D}_{i}} B_{j, \alpha}\right)=O\left(B_{i, \alpha}\right)
$$

in $B_{x_{i, \alpha}}\left(4 \delta_{i} r_{i, \alpha}\right) \backslash \bigcup_{j \in \mathscr{D}_{i}} B_{x_{j, \alpha}}\left(\frac{1}{10} R_{i, j} s_{j, i, \alpha}\right)$, and so, in particular, in $\Omega_{i, \alpha}$.

Proof. Let $x_{\alpha} \in B_{x_{i, \alpha}}\left(4 \delta_{i} r_{i, \alpha}\right) \backslash \bigcup_{j \in \mathscr{D}_{i}} B_{x_{j, \alpha}}\left(\frac{1}{10} R_{i, j} s_{j, i, \alpha}\right)$. Thanks to Proposition 6.1 we can write

$$
u_{\alpha}\left(x_{\alpha}\right)=u_{\infty}\left(x_{\alpha}\right)+\varepsilon_{\alpha}\left\|u_{\infty}\right\|_{\infty}+O\left(\mu_{\alpha}^{(n-2) / 2}\right)+\sum_{j=1}^{N}\left(\Lambda_{j}+o(1)\right) B_{j, \alpha}\left(x_{\alpha}\right) .
$$

By the definition of $r_{i, \alpha}$, we know that $r_{i, \alpha}^{2} \leq \mu_{i, \alpha}$ if $u_{\infty} \not \equiv 0$ so that

$$
u_{\infty}\left(x_{\alpha}\right)+\varepsilon_{\alpha}\left\|u_{\infty}\right\|_{\infty}=\mu_{i, \alpha}^{(n-2) / 2} r_{i, \alpha}^{2-n} \begin{cases}0 & \text { if } u_{\infty} \equiv 0, \\ \left(\lim _{\alpha \rightarrow+\infty} r_{i, \alpha}^{n-2} \mu_{i, \alpha}^{1-(n / 2)}\right) u_{\infty}\left(x_{i}\right)+o(1) & \text { if } u_{\infty} \neq \equiv .\end{cases}
$$


We now estimate $B_{j, \alpha}\left(x_{\alpha}\right)$. Assume first that $j \notin \mathscr{C}_{i}$ and $j \neq i$. As one can check with a little bit of work from the above definitions, if $r_{i, \alpha} \rightarrow 0$ as $\alpha \rightarrow+\infty$, then

$$
B_{j, \alpha}\left(x_{\alpha}\right)=\mu_{i, \alpha}^{(n-2) / 2} r_{i, \alpha}^{2-n} \Lambda_{i, j, \alpha},
$$

where

$$
\Lambda_{i, j, \alpha}= \begin{cases}\left(\frac{n(n-2)}{\left|z-z_{i, j}\right|^{2}} \frac{\mu_{j, \alpha}}{\mu_{i, \alpha}}\right)^{(n-2) / 2}+o(1) & \text { if } j \in \mathscr{A}_{i} \cap \mathscr{B}_{i} \text { and } i \in \mathscr{A}_{j}, \\ r_{i, \alpha}^{n-2} / s_{i, j, \alpha}^{n-2}+o(1) & \text { if } j \in \mathscr{A}_{i} \backslash \mathscr{B}_{i} \text { or } j \in \mathscr{A}_{i} \cap \mathscr{B}_{i} \text { and } i \notin \mathscr{A}_{j}, \\ o(1) & \text { if } j \in \mathscr{A}_{i}^{c} \backslash \mathscr{C}_{i},\end{cases}
$$

where, up to a subsequence,

$$
z=\lim _{\alpha \rightarrow+\infty} r_{i, \alpha}^{-1} \exp _{x_{i, \alpha}}^{-1}\left(x_{\alpha}\right) .
$$

Note that $z_{i, j} \neq 0$ if $j \in \mathscr{A}_{i} \cap \mathscr{P}_{i}$ and $i \in \mathscr{A}_{j}$. This is a direct consequence of the definition of the $s_{i, j, \alpha}$ 's and (8-6). Moreover, $\left|z-z_{i, j}\right| \geq 6 \delta_{i}$ in this case. As a consequence we have proved that

$$
\begin{aligned}
u_{\alpha}\left(x_{\alpha}\right)= & O\left(\mu_{\alpha}^{(n-2) / 2}\right)+o\left(\mu_{i, \alpha}^{(n-2) / 2} r_{i, \alpha}^{2-n}\right)+\left(\Lambda_{i}+o(1)\right) B_{i, \alpha}\left(x_{\alpha}\right) \\
& +\mu_{i, \alpha}^{(n-2) / 2} r_{i, \alpha}^{2-n} \Lambda(1)_{i, \alpha}+\mu_{i, \alpha}^{(n-2) / 2} r_{i, \alpha}^{2-n} \sum_{j \in \mathscr{A}_{i}} \Lambda(2)_{i, j, \alpha} \Lambda_{j}+\sum_{j \in \mathscr{C}_{i}}\left(\Lambda_{j}+o(1)\right) B_{j, \alpha}\left(x_{\alpha}\right),
\end{aligned}
$$

where

and

$$
\Lambda(1)_{i, \alpha}= \begin{cases}0 & \text { if } u_{\infty} \equiv 0 \\ \left(\lim _{\alpha \rightarrow+\infty} r_{i, \alpha}^{n-2} \mu_{i, \alpha}^{1-(n / 2)}\right) u_{\infty}\left(x_{i}\right) & \text { if } u_{\infty} \neq 0\end{cases}
$$

$$
\Lambda(2)_{i, j, \alpha}= \begin{cases}(n(n-2))^{(n-2) / 2} \lambda_{i, j} /\left|z-z_{i, j}\right|^{n-2} & \text { if } j \in \mathscr{B}_{i} \text { and } i \in \mathscr{A}_{j}, \\ \lim _{\alpha \rightarrow+\infty} r_{i, \alpha}^{n-2} / s_{i, j, \alpha}^{n-2} & \text { if } j \notin \mathscr{B}_{i} \text { or } i \notin \mathscr{A}_{j} .\end{cases}
$$

Let $j \in \mathscr{C}_{i}$. We claim that, up to a subsequence,

$$
\lim _{\alpha \rightarrow+\infty}\left(\frac{B_{j, \alpha}\left(x_{\alpha}\right)}{B_{i, \alpha}\left(x_{\alpha}\right)}\right)^{2 /(n-2)}=n(n-2) \lim _{\alpha \rightarrow+\infty} \frac{s_{j, i, \alpha}^{2}}{d_{g}\left(x_{j, \alpha}, x_{\alpha}\right)^{2}} .
$$

To prove (8-20), we first remark that $i \in \mathscr{A}_{j}$ since $j \in \mathscr{C}_{i}$ (and in particular $j \notin \mathscr{A}_{i}$ ). Thus, using (8-5), we obtain that

$$
\begin{aligned}
\left(\frac{B_{j, \alpha}\left(x_{\alpha}\right)}{B_{i, \alpha}\left(x_{\alpha}\right)}\right)^{2 /(n-2)} & =(1+o(1)) \mu_{j, \alpha} \mu_{i, \alpha}^{-1} d_{g}\left(x_{j, \alpha}, x_{\alpha}\right)^{-2}\left(n(n-2) \mu_{i, \alpha}^{2}+d_{g}\left(x_{i, \alpha}, x_{\alpha}\right)^{2}\right) \\
& =n(n-2) \frac{s_{j, i, \alpha}^{2}}{d_{g}\left(x_{j, \alpha}, x_{\alpha}\right)^{2}}+o(1)+O\left(\frac{\mu_{j, \alpha}}{\mu_{i, \alpha}} \frac{\left|d_{g}\left(x_{i, \alpha}, x_{\alpha}\right)^{2}-d_{g}\left(x_{i, \alpha}, x_{j, \alpha}\right)^{2}\right|}{d_{g}\left(x_{j, \alpha}, x_{\alpha}\right)^{2}}\right) .
\end{aligned}
$$

From the triangle inequality, we easily get

$$
\frac{\left|d_{g}\left(x_{i, \alpha}, x_{\alpha}\right)^{2}-d_{g}\left(x_{i, \alpha}, x_{j, \alpha}\right)^{2}\right|}{d_{g}\left(x_{j, \alpha}, x_{\alpha}\right)^{2}} \leq 1+2 \frac{d_{g}\left(x_{i, \alpha}, x_{j, \alpha}\right)}{d_{g}\left(x_{j, \alpha}, x_{\alpha}\right)}=O(1)+O\left(\frac{s_{j, i, \alpha}}{d_{g}\left(x_{j, \alpha}, x_{\alpha}\right)} \sqrt{\frac{\mu_{i, \alpha}}{\mu_{j, \alpha}}}\right),
$$

hence the estimate (8-20). Now, for $j \in \mathscr{C}_{i}$, we let $k \in \mathscr{D}_{i}$ be given by (8-13). By (8-20) it is easily checked that

$$
B_{j, \alpha}\left(x_{\alpha}\right)=O\left(B_{k, \alpha}\left(x_{\alpha}\right)\right) .
$$


Since

$$
\mu_{i, \alpha}^{(n-2) / 2} r_{i, \alpha}^{2-n}=O\left(B_{i, \alpha}\left(x_{\alpha}\right)\right),
$$

the first estimate in the lemma clearly holds true thanks to (8-19) and (8-21). Here it can be noted that

$$
\mu_{\alpha}^{(n-2) / 2}=O\left(\mu_{i, \alpha}^{(n-2) / 2} r_{i, \alpha}^{2-n}\right)
$$

for all $i$. Applying (8-20) again we easily obtain the second estimate in the lemma. This ends the proof of Lemma 8.1.

Now we prove that the following elliptic type lemma holds true. Lemma 8.2 provides estimates on the $u_{\alpha}$ 's and $\nabla u_{\alpha}$ 's in small regions around the blow-up points $x_{i, \alpha}$.

Lemma 8.2. There exists $C>0$ such that, up to a subsequence,

$$
\left|u_{\alpha}\right| \leq C \mu_{i, \alpha}^{(n-2) / 2} r_{i, \alpha}^{2-n} \quad \text { and } \quad\left|\nabla u_{\alpha}\right| \leq C \mu_{i, \alpha}^{(n-2) / 2} r_{i, \alpha}^{1-n}
$$

in $B_{x_{i, \alpha}}\left(2 \delta_{i} r_{i, \alpha}\right) \backslash B_{x_{i, \alpha}}\left(\frac{1}{2} \delta_{i} r_{i, \alpha}\right)$. There also exists $C>0$ such that, up to a subsequence, for any $j \in \mathscr{D}_{i}$,

$$
\left|u_{\alpha}\right| \leq C \mu_{j, \alpha}^{(n-2) / 2} s_{j, i, \alpha}^{2-n} \quad \text { and } \quad\left|\nabla u_{\alpha}\right| \leq C \mu_{j, \alpha}^{(n-2) / 2} s_{j, i, \alpha}^{1-n}
$$

in $B_{x_{j, \alpha}}\left(5 R_{i, j} s_{j, i, \alpha}\right) \backslash B_{x_{j, \alpha}}\left(\frac{1}{5} R_{i, j} s_{j, i, \alpha}\right)$.

Proof. The lemma follows from standard elliptic theory and the estimates we proved in Lemma 8.1. Assuming first that $x_{\alpha} \in B_{x_{i, \alpha}}\left(4 \delta_{i} r_{i, \alpha}\right) \backslash B_{x_{i, \alpha}}\left(\frac{1}{4} \delta_{i} r_{i, \alpha}\right)$, we easily get from Lemma 8.1 that

$$
\left|u_{\alpha}\left(x_{\alpha}\right)\right|=O\left(\mu_{i, \alpha}^{(n-2) / 2} r_{i, \alpha}^{2-n}\right) .
$$

On the other hand, if we let $\tilde{u}_{\alpha}$ be given by $\tilde{u}_{\alpha}(x)=r_{i, \alpha}^{(n-2) / 2} u_{\alpha}\left(\exp _{x_{i, \alpha}}\left(r_{i, \alpha} x\right)\right)$, then

$$
\Delta_{\tilde{g}_{\alpha}} \tilde{u}_{\alpha}+r_{i, \alpha}^{2} \tilde{A}_{\alpha} \tilde{u}_{\alpha}=\left|\tilde{u}_{\alpha}\right|^{2^{\star}-2} \tilde{u}_{\alpha}
$$

where $\tilde{g}_{\alpha}=\left(\exp _{x_{i, \alpha}}^{\star} g\right)\left(r_{i, \alpha} x\right)$ and $\tilde{A}_{\alpha}(x)=A_{\alpha}\left(\exp _{x_{i, \alpha}}\left(r_{i, \alpha} x\right)\right)$. The first two estimates in Lemma 8.2 follow from (8-23) and (8-24) by standard elliptic theory. Similarly, if we assume that

$$
x_{\alpha} \in B_{x_{j, \alpha}}\left(10 R_{i, j} s_{j, i, \alpha}\right) \backslash B_{x_{j, \alpha}}\left(\frac{1}{10} R_{i, j} s_{j, i, \alpha}\right),
$$

noting that $s_{j, i, \alpha}=o\left(r_{i, \alpha}\right)$ in this case, we get from Lemma 8.1 and (8-20) that

$$
\left|u_{\alpha}\left(x_{\alpha}\right)\right|=O\left(\mu_{j, \alpha}^{(n-2) / 2} s_{j, i, \alpha}^{2-n}\right) .
$$

Letting $\hat{U}_{\alpha}$ be given by $\hat{U}_{\alpha}(x)=s_{j, i, \alpha}^{(n-2) / 2} u_{\alpha}\left(\exp _{x_{j, \alpha}}\left(s_{j, i, \alpha} x\right)\right)$, we also have

$$
\Delta_{\hat{g}_{\alpha}} \hat{\vartheta}_{\alpha}+s_{j, i, \alpha}^{2} \hat{A}_{\alpha} \hat{U}_{\alpha}=\left|\hat{U}_{\alpha}\right|^{2^{\star}-2} \hat{U}_{\alpha} \text {, }
$$

where $\hat{g}_{\alpha}=\left(\exp _{x_{j, \alpha}}^{\star} g\right)\left(s_{j, i, \alpha} x\right)$ and $\hat{A}_{\alpha}(x)=A_{\alpha}\left(\exp _{x_{j, \alpha}}\left(s_{j, i, \alpha} x\right)\right)$. The last two estimates in Lemma 8.2 follow from (8-25) and (8-26) here again by standard elliptic theory. This proves Lemma 8.2.

\section{Sharp asymptotics for the range of influence}

Our goal now is to prove the sharp asymptotics connecting the range of influence $r_{i, \alpha}$ of the blow-up points with the weights $\mu_{i, \alpha}$ of the bubbles in the decomposition of Proposition 4.2. This is the subject of Proposition 9.2. We adopt here the notations of the preceding section. In particular, $\left(A_{\alpha}\right)_{\alpha}$ is a sequence 
of $C^{1}$ maps from $M$ to $M_{p}^{s}(\mathbb{R})$ such that $A_{\alpha} \rightarrow A$ in $C^{1}(M)$ as $\alpha \rightarrow+\infty$ for some $C^{1}$ map $A$ from $M$ to $M_{p}^{s}(\mathbb{R})$ satisfying $(\mathrm{H})$, and we order the blow-up points in such a way that

$$
\mu_{\alpha}=\mu_{1, \alpha} \geq \cdots \geq \mu_{N, \alpha},
$$

where the $\mu_{i, \alpha}$ 's are the weights of the vector bubble $\left(\Re_{\alpha}^{i}\right)_{\alpha}$ in Proposition 4.2, and let the $r_{i, \alpha}$ 's be given by (8-3). First we prove:

Lemma 9.1. If $r_{i, \alpha}=o\left(\sqrt{\mu_{i, \alpha} / \mu_{\alpha}}\right)$, then, up to a subsequence,

$$
r_{i, \alpha}^{n-2} \mu_{i, \alpha}^{1-(n / 2)} \bigcup_{\alpha}\left(\exp _{x_{i, \alpha}}\left(r_{i, \alpha} z\right)\right) \rightarrow(n(n-2))^{(n-2) / 2}\left(\Lambda_{i}|z|^{2-n}+\mathscr{H}_{i}(z)\right)
$$

in $C_{\mathrm{loc}}^{2}\left(B_{0}\left(2 \delta_{i}\right) \backslash\{0\}\right)^{p}$ as $\alpha \rightarrow+\infty$, where

$$
\mathscr{H}_{i}(z)=\sum_{\substack{j \in \mathscr{A}_{i} \cap \mathscr{B}_{i} \\ i \in \mathscr{A}_{j}}} \frac{\lambda_{i, j} \Lambda_{j}}{\left|z-z_{i, j}\right|^{n-2}}+X_{i}
$$

is a smooth function in $B_{0}\left(2 \delta_{i}\right)$ satisfying that $\mathscr{H}_{i}(0) \neq 0$, the $\lambda_{i, j}$ 's are as in (8-7), $\delta_{i}$ is as in (8-10), and the $X_{i}$ 's are nonnegative vectors in $\mathbb{R}^{p}$.

Proof. Let $z \in B_{0}\left(3 \delta_{i}\right) \backslash\{0\}$ and set $x_{\alpha}=\exp _{x_{i, \alpha}}\left(r_{i, \alpha} z\right)$. Let also $\mathscr{W}_{\alpha}$ be given by

$$
\bar{u}_{\alpha}(x)=r_{i, \alpha}^{n-2} \mu_{i, \alpha}^{-(n-2) / 2} u_{\alpha}\left(\exp _{x_{i, \alpha}}\left(r_{i, \alpha} x\right)\right) .
$$

Then

$$
\Delta_{g_{\alpha}} \bar{u}_{\alpha}+r_{i, \alpha}^{2} \tilde{A}_{\alpha} \bar{u}_{\alpha}=\left(\frac{\mu_{i, \alpha}}{r_{i, \alpha}}\right)^{2}\left|\bar{u}_{\alpha}\right|^{2^{\star}-2} \bar{u}_{\alpha},
$$

where $g_{\alpha}=\left(\exp _{x_{i, \alpha}}^{\star} g\right)\left(r_{i, \alpha} x\right)$ and $\tilde{A}_{\alpha}(x)=A_{\alpha}\left(\exp _{x_{i, \alpha}}\left(r_{i, \alpha} x\right)\right)$. In particular, we get by (8-6), (8-19) and (8-20) that, if $r_{i, \alpha}^{2}=o\left(\mu_{i, \alpha} / \mu_{\alpha}\right)$, then

$$
\lim _{\alpha \rightarrow+\infty} r_{i, \alpha}^{n-2} \mu_{i, \alpha}^{1-\frac{n}{2}} u_{\alpha}\left(x_{\alpha}\right)=(n(n-2))^{(n-2) / 2}\left(\Lambda_{i}|z|^{2-n}+\mathscr{H}_{i}(z)\right),
$$

where $\mathscr{H}_{i}(z)$ is the sum of two terms:

$$
\begin{cases}0 & \text { if } u_{\infty} \equiv 0 \\ \left(\lim _{\alpha \rightarrow+\infty} r_{i, \alpha}^{n-2} \mu_{i, \alpha}^{1-(n / 2)}\right) u_{\infty}\left(x_{i}\right) & \text { if } u_{\infty} \neq 0\end{cases}
$$

and

$$
\sum_{j \in \mathscr{A}_{i}} \Lambda_{j} \begin{cases}(n(n-2))^{(n-2) / 2} \lambda_{i, j} /\left|z-z_{i, j}\right|^{n-2} & \text { if } j \in \mathscr{B}_{i} \text { and } i \in \mathscr{A}_{j}, \\ \lim _{\alpha \rightarrow+\infty} r_{i, \alpha}^{n-2} / s_{i, j, \alpha}^{n-2} & \text { if } j \notin \mathscr{B}_{i} \text { or } i \notin \mathscr{A}_{j} .\end{cases}
$$

As a remark, if $j \in \mathscr{A}_{i}$ and $i \in \mathscr{A}_{j}$, then $\mu_{i, \alpha} \sim \mu_{j, \alpha}$. In particular, $z_{i, j} \neq 0$ since, if not the case, we would get from the inequality $r_{i, \alpha} \leq s_{i, j, \alpha}$ that $r_{i, \alpha}=o\left(\mu_{i, \alpha}\right)$ and then that $d_{g}\left(x_{i, \alpha}, x_{j, \alpha}\right)=o\left(\mu_{i, \alpha}\right)$, a contradiction with the first equation in (4-5) of Proposition 4.2. By (9-1) and (9-2), standard elliptic theory gives the lemma, up to the proof that $\mathscr{H}_{i}(0) \neq 0$. Assume first that there exists $j \in \mathscr{A}_{i}$ such that $s_{i, j, \alpha}=r_{i, \alpha}$. Then in the term involving this $j$ in the above sum over $\mathscr{A}_{i}$ there is at least one line which is positive. Since all the other terms are nonnegative, this proves that $\mathscr{H}_{i}(0) \neq 0$. The other possibility 
is that $u_{\infty} \not \equiv 0$ and that $r_{i, \alpha}^{2}=\mu_{i, \alpha}$ so the first term in the definition of $\mathscr{H}_{i}$ is nonzero. Indeed, by the maximum principle, since

$$
\Delta_{g}\left|u_{\infty}\right|_{\Sigma}+\Lambda\left|u_{\infty}\right|_{\Sigma} \geq 0
$$

for some $\Lambda>0$, where $\left|u_{\infty}\right|_{\Sigma}=\sum_{i} u_{i, \infty}$ is the sum of the components of $u_{\infty}$, we get that $\left|u_{\infty}\right|_{\Sigma}>0$ in $M$ if $U_{\infty} \neq \equiv 0$. Then, here again, $\mathcal{H}_{i}(0) \neq 0$. Noting that the above two possibilities are the only two possibilities since our assumption on $r_{i, \alpha}$ clearly implies that $r_{i, \alpha} \rightarrow 0$ as $\alpha \rightarrow+\infty$, this ends the proof of Lemma 9.1.

As it can be checked from the above proof, we have an explicit formula for the $X_{i}$ 's in Lemma 9.1. We get that

$$
X_{i}=\left(\lim _{\alpha \rightarrow+\infty} r_{i, \alpha}^{n-2} \mu_{i, \alpha}^{1-(n / 2)}\right) u_{\infty}\left(x_{i}\right)+\sum_{j \in\left(\mathscr{A}_{i} \backslash \mathscr{\Re}_{i}\right) \cup \Theta_{i}}\left(\lim _{\alpha \rightarrow+\infty} \frac{r_{i, \alpha}}{s_{i, j, \alpha}}\right)^{n-2} \Lambda_{j},
$$

where we adopt the convention that the first term in the right-hand side of (9-3) is zero if $u_{\infty} \equiv 0$, that the second term is zero if $\left(\mathscr{A}_{i} \backslash \mathscr{B}_{i}\right) \cup \Theta_{i}=\varnothing$, and where $\Theta_{i}=\left\{j \in \mathscr{A}_{i}\right.$ s.t. $\left.i \notin \mathscr{A}_{j}\right\}$. Now, at this point, we can state Proposition 9.2 which establishes sharp asymptotics connecting the range of influence $r_{i, \alpha}$ of the blow-up points $x_{i, \alpha}$ to the weights $\mu_{i, \alpha}$ of the bubbles in the decomposition of Proposition 4.2.

Proposition 9.2. Let $(M, g)$ be a smooth compact Riemannian manifold of dimension $n \geq 4, p \geq 1$ be an integer, and $\left(A_{\alpha}\right)_{\alpha}$ be a sequence of $C^{1}$ maps from $M$ to $M_{p}^{s}(\mathbb{R})$ such that $A_{\alpha} \rightarrow A$ in $C^{1}(M)$ as $\alpha \rightarrow+\infty$ for some $C^{1}$ map A from $M$ to $M_{p}^{s}(\mathbb{R})$ satisfying $(\mathrm{H})$. Let also $\left(\cup_{\alpha}\right)_{\alpha}$ be an arbitrary bounded sequence in $H^{1}(M)$ of nonnegative solutions of (1-1) such that $\left\|u_{\alpha}\right\|_{\infty} \rightarrow+\infty$ as $\alpha \rightarrow+\infty$. Let $i \in\{1, \ldots, N\}$ and assume that, up to a subsequence, $r_{i, \alpha}=o\left(\sqrt{\mu_{i, \alpha} / \mu_{\alpha}}\right)$. Then

$$
\left(\left(A\left(x_{i}\right)-\frac{1}{6} S_{g}\left(x_{i}\right) \operatorname{Id}_{p}\right) \Lambda_{i}+o(1)\right) r_{i, \alpha}^{2} \ln \frac{1}{\mu_{i, \alpha}}=2 \mathscr{H}_{i}(0)+o(1)
$$

if $n=4$, and

$$
\begin{aligned}
\left(\left(A\left(x_{i}\right)-\frac{n-2}{4(n-1)} S_{g}\left(x_{i}\right) \operatorname{Id}_{p}\right)\right. & \left.\Lambda_{i}+o(1)\right) \mu_{i, \alpha}^{4-n} r_{i, \alpha}^{n-2} \\
& =\frac{n^{n-2}(n-2)^{n-1} \omega_{n-1}}{\int_{\mathbb{R}^{n}} u_{0}^{2} d x}\left(\mathscr{H}_{i}(0)+\frac{n-4}{2}\left\langle\Lambda_{i}, \mathscr{H}_{i}(0)\right\rangle_{\mathbb{R}^{p}} \Lambda_{i}\right)+o(1)
\end{aligned}
$$

if $n \geq 5$, where $\mathcal{H}_{i}$ is as in Lemma 9.1, the $r_{i, \alpha}$ 's are as in (8-3), and $u_{0}$ is given by (4-3). Moreover, $\left\langle\Lambda_{i}, \nabla \mathcal{H}_{i}(0)\right\rangle_{\mathbb{R}^{p}} \equiv 0$.

We prove Proposition 9.2 by reverse induction on $i$. We let $i \in\{1, \ldots, N\}$ be such that $\sqrt{\mu_{\alpha}} r_{i, \alpha}=$ $o\left(\sqrt{\mu_{i, \alpha}}\right)$ and, in case $i<N$, we assume that

$$
\text { for any } j=i+1, \ldots, N,(9-4) \text { and (9-5) hold for } j \text { if } \sqrt{\mu_{\alpha}} r_{j, \alpha}=o\left(\sqrt{\mu_{j, \alpha}}\right) \text {. }
$$

If $i=N$ we do not assume anything. Then we aim to prove that (9-4) and (9-5) hold true for $i$. As a remark it should be noted that we always have

$$
\mathscr{H}_{i}(0)+\frac{n-4}{2}\left\langle\Lambda_{i}, \mathscr{H}_{i}(0)\right\rangle_{\mathbb{R}^{p}} \Lambda_{i} \neq 0
$$


Let $i \in\{1, \ldots, N\}, i<N$, be arbitrary. Assuming $\left(\mathrm{H}_{i}\right)$, we get that for any $j \in \mathscr{D}_{i}$,

$$
s_{j, i, \alpha}^{2-n}= \begin{cases}O\left(-\ln \mu_{j, \alpha}\right) & \text { if } n=4, \\ O\left(\mu_{j, \alpha}^{4-n}\right) & \text { if } n \geq 5 .\end{cases}
$$

Indeed, if $j \in \mathscr{D}_{i}$, then $j>i$. Moreover, for any $j \in \mathscr{D}_{i}$, we have $i \in \mathscr{A}_{j}$, so $s_{j, i, \alpha} \geq r_{j, \alpha}$, and clearly $s_{j, i, \alpha}^{2}=o\left(\mu_{j, \alpha} \mu_{i, \alpha}^{-1}\right)=o\left(\mu_{j, \alpha} \mu_{\alpha}^{-1}\right)$. In particular, $\sqrt{\mu_{\alpha}} r_{j, \alpha}=o\left(\sqrt{\mu_{j, \alpha}}\right)$, and (9-7) is a direct consequence of $\left(\mathrm{H}_{i}\right)$, thanks to (9-6). Now we prove Proposition 9.2 in several steps. In the sequel we let $R_{t}(\alpha)$ represent any quantity such that

$$
R_{t}(\alpha)= \begin{cases}o\left(-\mu_{i, \alpha}^{2} \ln \mu_{i, \alpha}\right) & \text { if } n=4, \\ o\left(\mu_{i, \alpha}^{2}\right) & \text { if } n \geq 5 .\end{cases}
$$

The first step in the proof of Proposition 9.2 is as follows.

Step 1. Let $i \in\{1, \ldots, N\}$ be arbitrary. In case $i<N$, assume that $\left(\mathrm{H}_{i}\right)$ holds true. Let

$$
\mathscr{F}_{\alpha}=\left(64 \omega_{3}\left(\left\langle A\left(x_{i}\right) \Lambda_{i}, \Lambda_{i}\right\rangle_{\mathbb{R}^{p}}-\frac{1}{6} S_{g}\left(x_{i}\right)\right)+o(1)\right) \mu_{i, \alpha}^{2} \ln \frac{r_{i, \alpha}}{\mu_{i, \alpha}}+o\left(-\mu_{i, \alpha}^{2} \ln \mu_{i, \alpha}\right)
$$

if $n=4$, and

$$
\mathscr{F}_{\alpha}=\left(\left(\left\langle A\left(x_{i}\right) \Lambda_{i}, \Lambda_{i}\right\rangle_{\mathbb{R}^{p}}-\frac{n-2}{4(n-1)} S_{g}\left(x_{i}\right)\right) \int_{\mathbb{R}^{n}} u_{0}^{2} d x+o(1)\right) \mu_{i, \alpha}^{2}
$$

if $n \geq 5$. Then we have, up to passing to a subsequence,

$$
\mathscr{F}_{\alpha}=\left(\frac{1}{2} n^{n-2}(n-2)^{n} \omega_{n-1}\left\langle\Lambda_{i}, \mathscr{H}_{i}(0)\right\rangle_{\mathbb{R}^{p}}+o(1)\right) \mu_{i, \alpha}^{n-2} r_{i, \alpha}^{2-n}
$$

if $\sqrt{\mu_{\alpha}} r_{i, \alpha}=o\left(\sqrt{\mu_{i, \alpha}}\right)$, and $\mathscr{F}_{\alpha}=O\left(\mu_{i, \alpha}^{(n-2) / 2} \mu_{\alpha}^{(n-2) / 2}\right)$ otherwise, where $\mathscr{H}_{i}$ is as in Lemma 9.1, the $r_{i, \alpha}$ 's are as in (8-3), and $u_{0}$ is as in (4-3).

Proof of Step 1. We apply the Pohozaev identity (7-2) of Proposition 7.1 in Section 7 to $u_{\alpha}$ in $\Omega_{i, \alpha}$ with $X=X^{\alpha}$ given by

$$
X^{\alpha}(x)=\left(1-\frac{1}{6(n-1)} R c_{g}^{\sharp}(x)\left(\nabla f_{\alpha}(x), \nabla f_{\alpha}(x)\right)\right) \nabla f_{\alpha}(x),
$$

where $f_{\alpha}(x)=\frac{1}{2} d_{g}\left(x_{i, \alpha}, x\right)^{2}$, and $R c_{g}^{\sharp}$ is the $(0,2)$-tensor field we get from the $(2,0)$-Ricci tensor $R c_{g}$ due to the musical isomorphism. We obtain

$$
\begin{aligned}
& \int_{\Omega_{i, \alpha}}\left\langle A_{\alpha} u_{\alpha}, X^{\alpha}\left(\nabla u_{\alpha}\right)\right\rangle_{\mathbb{R}^{p}} d v_{g} \\
& +\frac{n-2}{4 n} \int_{\Omega_{i, \alpha}}\left(\Delta_{g}\left(\operatorname{div}_{g} X^{\alpha}\right)\right)\left|u_{\alpha}\right|^{2} d v_{g}+\frac{n-2}{2 n} \int_{\Omega_{i, \alpha}}\left(\operatorname{div}_{g} X^{\alpha}\right)\left\langle A_{\alpha} u_{\alpha}, u_{\alpha}\right\rangle_{\mathbb{R}^{p}} d v_{g} \\
& =Q_{\alpha}-\sum_{j \in \mathscr{I}_{i}} Q_{\alpha}^{j}+R_{1, \alpha}+R_{2, \alpha}-\sum_{j \in \mathscr{D}_{i}} R_{2, \alpha}^{j},
\end{aligned}
$$


where, if $v=v_{\alpha}$ stands for the unit outer normal to $\partial B_{x_{i, \alpha}}\left(\delta_{i} r_{i, \alpha}\right)$, the $Q_{\alpha}$ 's are given by

$$
\begin{aligned}
Q_{\alpha}=\frac{n-2}{2 n} \int_{\partial B_{x_{i, \alpha}}\left(\delta_{i} r_{i, \alpha}\right)}\left(\operatorname{div}_{g} X^{\alpha}\right)\left\langle\partial_{\nu} \mathcal{U}_{\alpha}, u_{\alpha}\right\rangle_{\mathbb{R}^{p}} d \sigma_{g} & \\
& -\int_{\partial B_{x_{i, \alpha}}\left(\delta_{i} r_{i, \alpha}\right)}\left(\frac{1}{2} X^{\alpha}(v)\left|\nabla u_{\alpha}\right|^{2}-\left\langle X^{\alpha}\left(\nabla u_{\alpha}\right), \partial_{\nu} u_{\alpha}\right\rangle_{\mathbb{R}^{p}}\right) d \sigma_{g},
\end{aligned}
$$

the $Q_{\alpha}^{j}$ 's are given by

$$
\begin{aligned}
Q_{\alpha}^{j}=\frac{n-2}{2 n} \int_{\partial \Omega_{i, j, \alpha}}\left(\operatorname{div}_{g} X^{\alpha}\right)\left\langle\partial_{\nu} u_{\alpha}, u_{\alpha}\right\rangle_{\mathbb{R}^{p}} d \sigma_{g} & \\
& -\int_{\partial \Omega_{i, j, \alpha}}\left(\frac{1}{2} X^{\alpha}(\nu)\left|\nabla u_{\alpha}\right|^{2}-\left\langle X^{\alpha}\left(\nabla u_{\alpha}\right), \partial_{\nu} u_{\alpha}\right\rangle_{\mathbb{R}^{p}}\right) d \sigma_{g},
\end{aligned}
$$

where $\Omega_{i, j, \alpha}$ is as in (8-15), the $R_{1, \alpha}$ 's are given by

$$
R_{1, \alpha}=-\int_{\Omega_{i, \alpha}}\left(T_{\nabla} X^{\alpha}\right) u_{\alpha} d v_{g}
$$

where $\left(T_{\nabla} X\right)_{\varkappa}=\sum_{i=1}^{p} S_{X}^{\sharp}\left(\nabla u_{i}, \nabla u_{i}\right)$ and $S_{X}$ is as in (7-1), the $R_{2, \alpha}$ 's are given by

$$
R_{2, \alpha}=\frac{n-2}{2 n} \int_{\partial B_{x_{i, \alpha}}\left(\delta_{i} r_{i, \alpha}\right)} X^{\alpha}(v)\left|u_{\alpha}\right|^{2^{\star}} d \sigma_{g}-\frac{n-2}{4 n} \int_{\partial B_{x_{i, \alpha}}\left(\delta_{i} r_{i, \alpha}\right)}\left(\partial_{\nu}\left(\operatorname{div}_{g} X^{\alpha}\right)\right)\left|u_{\alpha}\right|^{2} d \sigma_{g},
$$

and the $R_{2, \alpha}^{j}$ 's are given by

$$
R_{2, \alpha}^{j}=\frac{n-2}{2 n} \int_{\partial \Omega_{i, j, \alpha}} X^{\alpha}(v)\left|u_{\alpha}\right|^{2^{\star}} d \sigma_{g}-\frac{n-2}{4 n} \int_{\partial \Omega_{i, j, \alpha}}\left(\partial_{\nu}\left(\operatorname{div}_{g} X^{\alpha}\right)\right)\left|u_{\alpha}\right|^{2} d \sigma_{g} .
$$

Note that $\mathscr{D}_{i}=\varnothing$ if $i=N$. Thanks to the expression of the $X^{\alpha}$ 's in (9-9) we have the estimates

$$
\begin{gathered}
\left|X^{\alpha}(x)\right|=O\left(d_{g}\left(x_{i, \alpha}, x\right)\right), \\
\operatorname{div}_{g} X^{\alpha}(x)-n=O\left(d_{g}\left(x_{i, \alpha}, x\right)^{2}\right), \\
\left|\nabla\left(\operatorname{div}_{g} X^{\alpha}\right)(x)\right|=O\left(d_{g}\left(x_{i, \alpha}, x\right)\right), \\
\Delta_{g}\left(\operatorname{div}_{g} X^{\alpha}\right)(x)=\frac{n}{n-1} S_{g}\left(x_{i, \alpha}\right)+O\left(d_{g}\left(x_{i, \alpha}, x\right)\right) .
\end{gathered}
$$

In what follows we estimate the different terms involved in (9-10). We start with estimates on the $Q_{\alpha}^{j}$ 's and $R_{2, \alpha}^{j}$ 's in (9-12) and (9-15). Since

$$
d_{g}\left(x_{i, \alpha}, x\right) \leq d_{g}\left(x_{i, \alpha}, x_{j, \alpha}\right)+R_{i, j} s_{j, i, \alpha}=O\left(\sqrt{\frac{\mu_{i, \alpha}}{\mu_{j, \alpha}}} s_{j, i, \alpha}\right)
$$

on $\partial \Omega_{i, j, \alpha}$, we obtain from Lemma 8.2, (9-7) and (9-16) that

$$
Q_{\alpha}^{j}+R_{2, \alpha}^{j}=O\left(\sqrt{\frac{\mu_{i, \alpha}}{\mu_{j, \alpha}}} \mu_{j, \alpha}^{n-2} s_{j, i, \alpha}^{2-n}\right)=R_{t}(\alpha),
$$

where $R_{t}(\alpha)$ is as in (9-8). Now we estimate the $R_{2, \alpha}$ 's in (9-14). Still from Lemma 8.2, we obtain by direct computations, using (8-6) and (9-16), that

$$
R_{2, \alpha}=O\left(\mu_{i, \alpha}^{n-2}\right)+o\left(\mu_{i, \alpha}^{n-2} r_{i, \alpha}^{2-n}\right) .
$$


Concerning the right-hand side of (9-10) it remains to estimate the $Q_{\alpha}$ 's in (9-11) and the $R_{1, \alpha}$ 's in (9-13). We start with estimates for the $R_{1, \alpha}$ 's. We remark that $S_{X^{\alpha}}^{\sharp}=O\left(d_{g}\left(x_{i, \alpha}, x\right)^{2}\right)$ and that

$$
\left(T_{\nabla} X^{\alpha}\right)_{\mathscr{B}_{\alpha}^{i}}=O\left(d_{g}\left(x_{i, \alpha}, x\right)^{3}\left|\nabla B_{i, \alpha}\right|^{2}\right) .
$$

In particular, we can write

$$
\begin{aligned}
R_{1, \alpha}=O\left(\int_{\Omega_{i, \alpha}} d_{g}\left(x_{i, \alpha}, x\right)^{3}\left|\nabla B_{i, \alpha}\right|^{2} d v_{g}\right) & +O\left(\int_{\Omega_{i, \alpha}} d_{g}\left(x_{i, \alpha}, x\right)^{2}\left|\nabla B_{i, \alpha}\right|\left|\nabla\left(u^{\alpha}-B_{i, \alpha} \Lambda_{i}\right)\right| d v_{g}\right) \\
& +O\left(\int_{\Omega_{i, \alpha}} d_{g}\left(x_{i, \alpha}, x\right)^{2}\left|\nabla\left(u^{\alpha}-B_{i, \alpha} \Lambda_{i}\right)\right|^{2} d v_{g}\right) .
\end{aligned}
$$

Direct computations lead to

$$
\int_{\Omega_{i, \alpha}} d_{g}\left(x_{i, \alpha}, x\right)^{2+\kappa}\left|\nabla B_{i, \alpha}\right|^{2} d v_{g}= \begin{cases}O_{\kappa}\left(-\mu_{i, \alpha}^{2} \ln \mu_{i, \alpha}\right) & \text { if } n=4, \\ O_{\kappa}\left(\mu_{i, \alpha}^{2}\right) & \text { if } n \geq 5,\end{cases}
$$

where $O_{\kappa}=O$ if $\kappa=0$, and $O_{\kappa}=o$ if $\kappa=1$. Integrating by parts and using Lemma 8.1, Lemma 8.2, and (9-7), we can write

$$
\begin{aligned}
& \int_{\Omega_{i, \alpha}} d_{g}\left(x_{i, \alpha}, x\right)^{2}\left|\nabla\left(u_{\alpha}-B_{i, \alpha} \Lambda_{i}\right)\right|^{2} d v_{g} \\
& =O\left(\int_{\partial \Omega_{i, \alpha}}\left|u_{\alpha}-B_{i, \alpha} \Lambda_{i}\right| d_{g}\left(x_{i, \alpha}, x\right)^{2}\left|\nabla\left(u_{\alpha}-B_{i, \alpha} \Lambda_{i}\right)\right| d \sigma_{g}\right)+O\left(\int_{\partial \Omega_{i, \alpha}} d_{g}\left(x_{i, \alpha}, x\right)\left|u_{\alpha}-B_{i, \alpha} \Lambda_{i}\right|^{2} d \sigma_{g}\right) \\
& \quad+O\left(\int_{\Omega_{i, \alpha}}\left|u_{\alpha}-B_{i, \alpha} \Lambda_{i}\right|^{2} d v_{g}\right)+\int_{\Omega_{i, \alpha}} d_{g}\left(x_{i, \alpha}, x\right)^{2}\left|u_{\alpha}-B_{i, \alpha} \Lambda_{i}, \Delta_{g}\left(u_{\alpha}-B_{i, \alpha} \Lambda_{i}\right)\right|_{\mathbb{R}^{p}} d v_{g},
\end{aligned}
$$

and then

$$
\begin{aligned}
\int_{\Omega_{i, \alpha}} d_{g}\left(x_{i, \alpha}, x\right)^{2} \mid \nabla & \left.\left(U_{\alpha}-B_{i, \alpha} \Lambda_{i}\right)\right|^{2} d v_{g} \\
& =\int_{\Omega_{i, \alpha}} d_{g}\left(x_{i, \alpha}, x\right)^{2}\left\langle u_{\alpha}-B_{i, \alpha} \Lambda_{i},\left.\Delta_{g}\left(\cup_{\alpha}-B_{i, \alpha} \Lambda_{i}\right)\right|_{\mathbb{R}^{p}} d v_{g}+o\left(\mu_{i, \alpha}^{n-2} r_{i, \alpha}^{2-n}\right)+R_{t}(\alpha),\right.
\end{aligned}
$$

where $R_{t}(\alpha)$ is as in (9-8). It remains to remark that thanks to the equations satisfied by the $u_{\alpha}$ 's, and the expression of $\Delta_{g}$ in geodesic polar coordinates, we have

$$
\begin{aligned}
& \int_{\Omega_{i, \alpha}} d_{g}\left(x_{i, \alpha}, x\right)^{2}\left|u_{\alpha}-B_{i, \alpha} \Lambda_{i}, \Delta_{g}\left(u_{\alpha}-B_{i, \alpha} \Lambda_{i}\right)\right|_{\mathbb{R}^{p}} d v_{g} \\
& =O\left(\int_{\Omega_{i, \alpha}} d_{g}\left(x_{i, \alpha}, x\right)^{2}\left|u_{\alpha}-B_{i, \alpha} \Lambda_{i}\right|\left(\left|u_{\alpha}\right|^{2^{\star}-1}+B_{i, \alpha}^{2^{\star}-1}\right) d v_{g}\right) \\
& \quad+O\left(\int_{\Omega_{i, \alpha}} d_{g}\left(x_{i, \alpha}, x\right)^{2}\left|u_{\alpha}-B_{i, \alpha} \Lambda_{i}\right|\left|u_{\alpha}\right| d v_{g}\right)+O\left(\int_{\Omega_{i, \alpha}} d_{g}\left(x_{i, \alpha}, x\right)^{3}\left|\cup_{\alpha}-B_{i, \alpha} \Lambda_{i}\right|\left|\nabla B_{i, \alpha}\right| d v_{g}\right),
\end{aligned}
$$

so that, by Lemma 8.1, using Hölder's inequalities,

$$
R_{1, \alpha}=o\left(\mu_{i, \alpha}^{n-2} r_{i, \alpha}^{2-n}\right)+R_{t}(\alpha),
$$


where $R_{t}(\alpha)$ is as in (9-8). Still concerning the right-hand side of (9-10) it remains to estimate the $Q_{\alpha}$ 's in (9-11). Thanks to Lemma 8.2 and Lemma 9.1, we get by simple computations that

$$
Q_{\alpha}=\left(-\frac{1}{2} n^{n-2}(n-2)^{n} \omega_{n-1}\left\langle\Lambda_{i}, \mathscr{H}_{i}(0)\right\rangle_{\mathbb{R}^{p}}+o(1)\right) \mu_{i, \alpha}^{n-2} r_{i, \alpha}^{2-n}+O\left(\mu_{i, \alpha}^{n-2}\right),
$$

if $r_{i, \alpha}=o\left(\sqrt{\mu_{i, \alpha} / \mu_{\alpha}}\right)$, and $Q_{\alpha}=O\left(\mu_{i, \alpha}^{(n-2) / 2} \mu_{\alpha}^{(n-2) / 2}\right)$ otherwise. Now we concentrate on the left-hand side of (9-10). Writing $A_{\alpha}(x)=A_{\alpha}\left(x_{i, \alpha}\right)+O\left(d_{g}\left(x_{i, \alpha}, x\right)\right)$, we get

$\int_{\Omega_{i, \alpha}}\left\langle A_{\alpha} \cup_{\alpha}, X^{\alpha}\left(\nabla u_{\alpha}\right)\right\rangle_{\mathbb{R}^{p}} d v_{g}=\sum_{j, k=1}^{p} A_{j k}^{\alpha}\left(x_{i, \alpha}\right) \int_{\Omega_{i, \alpha}} u_{j, \alpha} X^{\alpha}\left(\nabla u_{\alpha}\right)_{k} d v_{g}+O\left(\int_{\Omega_{i, \alpha}} d_{g}\left(x_{i, \alpha}, x\right)^{2}\left|u_{\alpha}\right|\left|\nabla u_{\alpha}\right| d v_{g}\right)$.

Using the Cauchy-Schwarz inequality, we can write

$$
\int_{\Omega_{i, \alpha}} d_{g}\left(x_{i, \alpha}, x\right)^{2}\left|u_{\alpha}\right|\left|\nabla u_{\alpha}\right| d v_{g} \leq \Pi_{j=0}^{1}\left(\int_{\Omega_{i, \alpha}} d_{g}\left(x_{i, \alpha}, x\right)^{3-2 j}\left|\nabla^{1-j} u_{\alpha}\right|^{2} d v_{g}\right)^{1 / 2} .
$$

Using Lemma 8.1 it is easily checked that

$$
\int_{\Omega_{i, \alpha}} d_{g}\left(x_{i, \alpha}, x\right)\left|u_{\alpha}\right|^{2} d v_{g}=R_{t}(\alpha),
$$

where $R_{t}(\alpha)$ is as in (9-8). We integrate by parts and use the equations satisfied by the $\varkappa_{\alpha}$ 's, together with Lemma 8.1, Lemma 8.2, and (9-7), to obtain

$$
\begin{aligned}
\int_{\Omega_{i, \alpha}} d_{g}\left(x_{i, \alpha}, x\right)^{3}\left|\nabla u_{\alpha}\right|^{2} d v_{g}= & O\left(\int_{\partial \Omega_{i, \alpha}} d_{g}\left(x_{i, \alpha}, x\right)^{3}\left|\nabla u_{\alpha}\right|\left|u_{\alpha}\right| d \sigma_{g}\right) \\
& +O\left(\int_{\partial \Omega_{i, \alpha}} d_{g}\left(x_{i, \alpha}, x\right)^{2}\left|u_{\alpha}\right|^{2} d \sigma_{g}\right)+O\left(\int_{\Omega_{i, \alpha}} d_{g}\left(x_{i, \alpha}, x\right)^{3}\left|u_{\alpha}\right|^{2^{\star}} d v_{g}\right) \\
& +O\left(\int_{\Omega_{i, \alpha}} d_{g}\left(x_{i, \alpha}, x\right)\left|u_{\alpha}\right|^{2} d v_{g}\right),
\end{aligned}
$$

and then that

$$
\int_{\Omega_{i, \alpha}} d_{g}\left(x_{i, \alpha}, x\right)^{3}\left|\nabla u_{\alpha}\right|^{2} d v_{g}=o\left(\mu_{i, \alpha}^{n-2} r_{i, \alpha}^{2-n}\right)+R_{t}(\alpha),
$$

where $R_{t}(\alpha)$ is as in (9-8). Thus we get that

$$
\int_{\Omega_{i, \alpha}}\left\langle A_{\alpha} \cup_{\alpha}, X^{\alpha}\left(\nabla \mathcal{U}_{\alpha}\right)\right\rangle_{\mathbb{R}^{p}} d v_{g}=\sum_{j, k=1}^{p} A_{j k}^{\alpha}\left(x_{i, \alpha}\right) \int_{\Omega_{i, \alpha}} u_{j, \alpha} X^{\alpha}\left(\nabla u_{\alpha}\right)_{k} d v_{g}+o\left(\mu_{i, \alpha}^{n-2} r_{i, \alpha}^{2-n}\right)+R_{t}(\alpha) .
$$

Integrating by parts again, and estimating the different terms as above, it is easily checked that we actually have

$$
\int_{\Omega_{i, \alpha}}\left\langle A_{\alpha} u_{\alpha}, X^{\alpha}\left(\nabla u_{\alpha}\right)\right\rangle_{\mathbb{R}^{p}} d v_{g}=-\frac{n}{2} \sum_{j, k=1}^{p} A_{j k}^{\alpha}\left(x_{i, \alpha}\right) \int_{\Omega_{i, \alpha}} u_{j, \alpha} u_{k, \alpha} d v_{g}+o\left(\mu_{i, \alpha}^{n-2} r_{i, \alpha}^{2-n}\right)+R_{t}(\alpha),
$$


where $R_{t}(\alpha)$ is as in (9-8). Proceeding as above, thanks to (9-16), one finally gets that

$$
\begin{aligned}
& \int_{\Omega_{i, \alpha}}\left\langle A_{\alpha} u_{\alpha}, X^{\alpha}\left(\nabla u_{\alpha}\right)\right\rangle_{\mathbb{R}^{p}} d v_{g}+\frac{n-2}{4 n} \int_{\Omega_{i, \alpha}}\left(\Delta_{g}\left(\operatorname{div}_{g} X^{\alpha}\right)\right)\left|u_{\alpha}\right|^{2} d v_{g} \\
& \quad+\frac{n-2}{2 n} \int_{\Omega_{i, \alpha}}\left(\operatorname{div}_{g} X^{\alpha}\right)\left\langle A_{\alpha} u_{\alpha}, \cup_{\alpha}\right\rangle_{\mathbb{R}^{p}} d v_{g} \\
& =-\sum_{j, k=1}^{p} A_{j k}^{\alpha}\left(x_{i, \alpha}\right) \int_{\Omega_{i, \alpha}} u_{j, \alpha} u_{k, \alpha} d v_{g}+\frac{n-2}{4(n-1)} S_{g}\left(x_{i, \alpha}\right) \int_{\Omega_{i, \alpha}}\left|u_{\alpha}\right|^{2} d v_{g}+o\left(\mu_{i, \alpha}^{n-2} r_{i, \alpha}^{2-n}\right)+R_{t}(\alpha),
\end{aligned}
$$

where $R_{t}(\alpha)$ is as in (9-8). We have

$$
\int_{\Omega_{i, j, \alpha}} B_{i, \alpha}^{2} d v_{g}=R_{t}(\alpha) \text { for all } j \in \mathscr{D}_{i}
$$

Indeed, if $d_{g}\left(x_{i, \alpha}, x_{j, \alpha}\right) / s_{j, i, \alpha} \rightarrow+\infty$ as $\alpha \rightarrow+\infty$, then

$$
\int_{B_{x_{j, \alpha}}\left(R_{i, j} s_{j, i, \alpha}\right)} B_{i, \alpha}^{2} d v_{g}=O\left(s_{j, i, \alpha}^{n} B_{i, \alpha}\left(x_{j, \alpha}\right)^{2}\right)=O\left(\mu_{j, \alpha}^{n-2} s_{j, i, \alpha}^{4-n}\right)=R_{t}(\alpha),
$$

thanks to (9-7), and if $d_{g}\left(x_{i, \alpha}, x_{j, \alpha}\right)=O\left(s_{j, i, \alpha}\right)$, then $s_{j, i, \alpha}=o\left(\mu_{i, \alpha}\right)$ and

$$
\int_{B_{x_{j, \alpha}}\left(R_{i, j} s_{j, i, \alpha}\right)} B_{i, \alpha}^{2} d v_{g}=O\left(\mu_{i, \alpha}^{2-n} s_{j, i, \alpha}^{n}\right)=o\left(\mu_{i, \alpha}^{2}\right) .
$$

Clearly, (9-23) follows from these two equations. Plugging (9-23) into (9-22), we get from Lemma 8.1 that

$$
\begin{gathered}
\int_{\Omega_{i, \alpha}}\left\langle A_{\alpha} u_{\alpha}, X^{\alpha}\left(\nabla u_{\alpha}\right)\right\rangle_{\mathbb{R}^{p}} d v_{g}+\frac{n-2}{4 n} \int_{\Omega_{i, \alpha}}\left(\Delta_{g}\left(\operatorname{div}_{g} X^{\alpha}\right)\right)\left|u_{\alpha}\right|^{2} d v_{g}+\frac{n-2}{2 n} \int_{\Omega_{i, \alpha}}\left(\operatorname{div}_{g} X^{\alpha}\right)\left\langle A_{\alpha} \cup_{\alpha}, \cup_{\alpha}\right\rangle_{\mathbb{R}^{p}} d v_{g} \\
=-\left(\left\langle A\left(x_{i}\right) \Lambda_{i}, \Lambda_{i}\right\rangle_{\mathbb{R}^{p}}-\frac{n-2}{4(n-1)} S_{g}\left(x_{i}\right)+o(1)\right) \int_{B_{x_{i, \alpha}}\left(\delta_{i} r_{i, \alpha}\right)} B_{i, \alpha}^{2} d v_{g}+o\left(\mu_{i, \alpha}^{n-2} r_{i, \alpha}^{2-n}\right)+R_{t}(\alpha) . \quad(9-24)
\end{gathered}
$$

We have

$$
\int_{B_{x_{i, \alpha}}\left(\delta_{i} r_{i, \alpha}\right)} B_{i, \alpha}^{2} d v_{g}= \begin{cases}64 \omega_{3} \mu_{i, \alpha}^{2} \ln \left(r_{i, \alpha} / \mu_{i, \alpha}\right)+o\left(-\mu_{i, \alpha}^{2} \ln \mu_{i, \alpha}\right) & \text { if } n=4, \\ \left(\int_{\mathbb{R}^{n}} u_{0}^{2} d x\right) \mu_{i, \alpha}^{2}+o\left(\mu_{i, \alpha}^{2}\right) & \text { if } n \geq 5,\end{cases}
$$

where $u_{0}$ is given by (4-3). Combining (9-10), (9-17)-(9-20), (9-24), and (9-25) yields the proof of Step 1.

Step 2. Let $i \in\{1, \ldots, N\}$ be arbitrary. In case $i<N$, assume that $\left(\mathrm{H}_{i}\right)$ holds. Let $\mathscr{K}_{\alpha}$ be given by

$$
\mathscr{K}_{\alpha}=\left(64 \omega_{3}\left(A\left(x_{i}\right) \Lambda_{i}-\frac{1}{6} S_{g}\left(x_{i}\right) \Lambda_{i}\right)+o(1)\right) \mu_{i, \alpha}^{2} \ln \frac{r_{i, \alpha}}{\mu_{i, \alpha}}+o\left(-\mu_{i, \alpha}^{2} \ln \mu_{i, \alpha}\right)
$$

in case $n=4$, and

$$
\mathscr{K}_{\alpha}=\left(\left(A\left(x_{i}\right) \Lambda_{i}-\frac{n-2}{4(n-1)} S_{g}\left(x_{i}\right) \Lambda_{i}\right) \int_{\mathbb{R}^{n}} u_{0}^{2} d x+o(1)\right) \mu_{i, \alpha}^{2}
$$


in case $n \geq 5$. Then, up to passing to a subsequence, we have

$$
\mathscr{K}_{\alpha}=\left(n^{n-2}(n-2)^{n-1} \omega_{n-1}\left(\mathscr{H}_{i}(0)+\frac{n-4}{2}\left\langle\mathscr{H}_{i}(0), \Lambda_{i}\right\rangle \Lambda_{i}\right)+o(1)\right) \mu_{i, \alpha}^{n-2} r_{i, \alpha}^{2-n},
$$

if $\sqrt{\mu_{\alpha}} r_{i, \alpha}=o\left(\sqrt{\mu_{i, \alpha}}\right)$, and $\mathcal{K}_{\alpha}=O\left(\mu_{i, \alpha}^{(n-2) / 2} \mu_{\alpha}^{(n-2) / 2}\right)$ otherwise, where $\mathscr{H}_{i}$ is as in Lemma 9.1, the $r_{i, \alpha}$ 's are as in (8-3), and $u_{0}$ is as in (4-3).

Proof of Step 2. We multiply the line $k$ of the system (1-1) by $u_{l, \alpha}$ and integrate over $\Omega_{i, \alpha}$. This leads to

$$
\int_{\Omega_{i, \alpha}} u_{l, \alpha} \Delta_{g} u_{k, \alpha} d v_{g}+\sum_{m=1}^{p} \int_{\Omega_{i, \alpha}} A_{k m}^{\alpha} u_{l, \alpha} u_{m, \alpha} d v_{g}=\int_{\Omega_{i, \alpha}}\left|u_{\alpha}\right|^{2^{\star}-2} u_{k_{\alpha}} u_{l, \alpha} d v_{g} .
$$

Let the $\Lambda_{i, k}$ 's, $k=1, \ldots, p$, be the components of $\Lambda_{i}$, and the $\mathscr{H}_{i, k}$ 's be the components of $\mathscr{H}_{i}$. We define $S_{k, l}^{\alpha}$ by

$$
S_{k, l}^{\alpha}=\left(n^{n-2}(n-2)^{n-1} \omega_{n-1}\left(\Lambda_{i, k} \mathscr{H}_{i, l}(0)-\Lambda_{i, l} \mathscr{H}_{i, k}(0)\right)+o(1)\right) \mu_{i, \alpha}^{n-2} r_{i, \alpha}^{2-n},
$$

if $r_{i, \alpha}=o\left(\sqrt{\mu_{i, \alpha} / \mu_{\alpha}}\right)$, and $S_{k, l}^{\alpha}=O\left(\mu_{i, \alpha}^{(n-2) / 2} \mu_{\alpha}^{(n-2) / 2}\right)$ otherwise. We also define $T_{k, l}^{\alpha}$ by

$$
T_{k, l}^{\alpha}= \begin{cases}\left(64 \omega_{3} W_{k, l}+o(1)\right) \mu_{i, \alpha}^{2} \ln \left(r_{i, \alpha} / \mu_{i, \alpha}\right)+o\left(-\mu_{i, \alpha}^{2} \ln \mu_{i, \alpha}\right) & \text { if } n=4, \\ \left(W_{k, l} \int_{\mathbb{R}^{n}} u_{0}^{2} d x+o(1)\right) \mu_{i, \alpha}^{2} & \text { if } n \geq 5,\end{cases}
$$

where

$$
W_{k, l}=\sum_{m=1}^{p}\left(A\left(x_{i}\right)_{l m} \Lambda_{i, k} \Lambda_{i, m}-A\left(x_{i}\right)_{k m} \Lambda_{i, l} \Lambda_{i, m}\right),
$$

and $u_{0}$ is given by (4-3). Integrating by parts, thanks to Lemma 8.2 and Lemma 9.1, we have

$$
\begin{aligned}
\int_{\Omega_{i, \alpha}} u_{l, \alpha} \Delta_{g} u_{k, \alpha} d v_{g} & =\int_{\Omega_{i, \alpha}} u_{k, \alpha} \Delta_{g} u_{l, \alpha} d v_{g}+\int_{\partial \Omega_{i, \alpha}}\left(u_{k, \alpha} \partial_{\nu} u_{l, \alpha}-u_{l, \alpha} \partial_{\nu} u_{k, \alpha}\right) d \sigma_{g} \\
& =\int_{\Omega_{i, \alpha}}\left|u_{\alpha}\right|^{2^{\star}-2} u_{k_{\alpha}} u_{l, \alpha} d v_{g}-\sum_{m=1}^{p} \int_{\Omega_{i, \alpha}} A_{l m}^{\alpha} u_{k, \alpha} u_{m, \alpha} d v_{g}+O\left(\sum_{j \in \mathscr{D}_{i}} \mu_{j, \alpha}^{n-2} s_{j, i, \alpha}^{2-n}\right)+S_{k, l}^{\alpha} .
\end{aligned}
$$

Now we write $A_{\alpha}(x)=A_{\alpha}\left(x_{i, \alpha}\right)+O\left(d_{g}\left(x_{i, \alpha}, x\right)\right)$. With similar estimates as in the proof of Step 1, thanks to (9-21), we get that

$$
\sum_{m=1}^{p} \int_{\Omega_{i, \alpha}} A_{l m}^{\alpha} u_{k, \alpha} u_{m, \alpha} d v_{g}-\sum_{m=1}^{p} \int_{\Omega_{i, \alpha}} A_{k m}^{\alpha} u_{l, \alpha} u_{m, \alpha} d v_{g}=o\left(\mu_{i, \alpha}^{n-2} r_{i, \alpha}^{2-n}\right)+T_{k, l}^{\alpha} .
$$

Coming back to (9-26) with all these estimates, thanks to (9-7), we obtain that $S_{k, l}^{\alpha}=T_{k, l}^{\alpha}$. In particular, $\sum_{k} S_{k, l}^{\alpha} \Lambda_{i, k}=\sum_{k} T_{k, l}^{\alpha} \Lambda_{i, k}$ and Step 2 follows from Step 1. This ends the proof of Step 2.

Conclusion of the proof of Proposition 9.2. Equations (9-4) and (9-5) follow from Step 2. It remains to prove that $\left\langle\Lambda_{i}, \nabla \mathscr{H}_{i}(0)\right\rangle_{\mathbb{R}^{p}} \equiv 0$. We assume here that $\sqrt{\mu_{\alpha}} r_{i, \alpha}=o\left(\sqrt{\mu_{i, \alpha}}\right)$. In particular, $r_{i, \alpha} \rightarrow 0$ as $\alpha \rightarrow+\infty$. Let $Y$ be an arbitrary 1 -form in $\mathbb{R}^{n}$. We apply once more the Pohozaev identity (7-2) to $u_{\alpha}$ in $\Omega_{i, \alpha}$. However, here we choose $X=X^{\alpha}$ to be given in the exponential chart at $x_{i, \alpha}$ by

$$
X_{\kappa}^{\alpha}=Y_{\kappa}-\frac{2}{3} R_{\kappa j k l}\left(x_{i, \alpha}\right) x^{j} x^{k} Y^{l},
$$


where $Y^{l}=Y_{l}$ for all $l$ and the $R_{\kappa j k l}$ are the components of the Riemann tensor $R m_{g}$ at $x_{i, \alpha}$ in the exponential chart. As is easily checked, still in geodesic normal coordinates at $x_{i, \alpha}$,

$$
\left(\nabla X^{\alpha}\right)_{\kappa j}=-\mathscr{R}_{\kappa j k l}\left(x_{i, \alpha}\right) x^{k} Y^{l}+O\left(|x|^{2}\right),
$$

so that $\operatorname{div}_{g}\left(X^{\alpha}\right)=O\left(|x|^{2}\right)$. Then, thanks to the symmetries of the Riemann tensor, we obtain with the Pohozaev identity that

$$
\begin{aligned}
& \int_{\partial \Omega_{i, \alpha}}\left(\frac{1}{2} X^{\alpha}(v)\left|\nabla u_{\alpha}\right|^{2}-\left\langle X^{\alpha}\left(\nabla u_{\alpha}\right), \partial_{\nu} u_{\alpha}\right\rangle_{\mathbb{R}^{p}}\right) d \sigma_{g}+\int_{\Omega_{i, \alpha}}\left\langle A_{\alpha} u_{\alpha}, X^{\alpha}\left(\nabla u_{\alpha}\right)\right\rangle_{\mathbb{R}^{p}} d v_{g} \\
& =O\left(\int_{\Omega_{i, \alpha}}\left|u_{\alpha}\right|^{2} d v_{g}\right)+O\left(\int_{\Omega_{i, \alpha}} d_{g}\left(x_{i, \alpha}, x\right)^{2}\left|\nabla u_{\alpha}\right|^{2} d v_{g}\right)+O\left(\int_{\partial \Omega_{i, \alpha}}\left|u_{\alpha}\right|^{2^{\star}} d \sigma_{g}\right) \\
& +O\left(\int_{\partial \Omega_{i, \alpha}}\left|u_{\alpha}\right|^{2} d \sigma_{g}\right)+O\left(\int_{\partial \Omega_{i, \alpha}}\left|\partial_{\nu} u_{\alpha}\right|\left|u_{\alpha}\right| d \sigma_{g}\right),
\end{aligned}
$$

Estimating the right-hand side of (9-27) via (9-7) and using Lemma 8.1 and Lemma 8.2, we get

$$
\int_{\partial \Omega_{i, \alpha}}\left(\frac{1}{2} X^{\alpha}(v)\left|\nabla u_{\alpha}\right|^{2}-\left\langle X^{\alpha}\left(\nabla u_{\alpha}\right), \partial_{\nu} u_{\alpha}\right\rangle_{\mathbb{R}^{p}}\right) d \sigma_{g}+\int_{\Omega_{i, \alpha}}\left\langle A_{\alpha} u_{\alpha}, X^{\alpha}\left(\nabla u_{\alpha}\right)\right\rangle_{\mathbb{R}^{p}} d v_{g}=\hat{R}_{t}(\alpha),
$$

where $\hat{R}_{t}(\alpha)$ is such that

$$
\hat{R}_{t}(\alpha)= \begin{cases}O\left(-\mu_{i, \alpha}^{2} \ln \mu_{i, \alpha}\right)+O\left(\mu_{i, \alpha}^{n-2} r_{i, \alpha}^{2-n}\right) & \text { if } n=4, \\ O\left(\mu_{i, \alpha}^{2}\right)+O\left(\mu_{i, \alpha}^{n-2} r_{i, \alpha}^{2-n}\right) & \text { if } n \geq 5 .\end{cases}
$$

Now we can write

$$
\int_{\Omega_{i, \alpha}}\left\langle A_{\alpha} \cup_{\alpha}, X^{\alpha}\left(\nabla u_{\alpha}\right)\right\rangle_{\mathbb{R}^{p}} d v_{g}=\sum_{k, l=1}^{p} A_{k l}^{\alpha}\left(x_{i, \alpha}\right) \int_{\Omega_{i, \alpha}} u_{k, \alpha} X^{\alpha}\left(\nabla u_{\alpha}\right)_{l} d v_{g}+O\left(T_{\alpha}\right),
$$

where

$$
T_{\alpha}=\int_{\Omega_{i, \alpha}} d_{g}\left(x_{i, \alpha}, x\right)\left|\nabla u_{\alpha}\right|\left|u_{\alpha}\right| d v_{g},
$$

obtaining

$$
\begin{aligned}
& \int_{\Omega_{i, \alpha}}\left\langle A_{\alpha} u_{\alpha}, X^{\alpha}\left(\nabla u_{\alpha}\right)\right\rangle_{\mathbb{R}^{p}} d v_{g} \\
& \quad=\frac{1}{2} \sum_{k, l=1}^{p} A_{k l}^{\alpha}\left(x_{i, \alpha}\right) \int_{\partial \Omega_{i, \alpha}} u_{k, \alpha} u_{l, \alpha} X^{\alpha}(v) d \sigma_{g}-\frac{1}{2} \sum_{k, l=1}^{p} A_{k l}^{\alpha}\left(x_{i, \alpha}\right) \int_{\Omega_{i, \alpha}} u_{k, \alpha} u_{l, \alpha} \operatorname{div}_{g}\left(X^{\alpha}\right) d v_{g}+O\left(T_{\alpha}\right) .
\end{aligned}
$$

As above, estimating the various terms in this equation, it follows that

$$
\int_{\Omega_{i, \alpha}}\left\langle A_{\alpha} u_{\alpha},\left.X^{\alpha}\left(\nabla u_{\alpha}\right)\right|_{\mathbb{R}^{p}} d v_{g}=\hat{R}_{t}(\alpha)\right.
$$

where $\hat{R}_{t}(\alpha)$ is as in (9-29). As a consequence, coming back to (9-28), thanks to (9-30), we get

$$
\int_{\partial \Omega_{i, \alpha}}\left(\frac{1}{2} X^{\alpha}(\nu)\left|\nabla \mathcal{U}_{\alpha}\right|^{2}-\left\langle X^{\alpha}\left(\nabla \mathcal{U}_{\alpha}\right), \partial_{\nu} \cup_{\alpha}\right\rangle_{\mathbb{R}^{p}}\right) d \sigma_{g}=\hat{R}_{t}(\alpha),
$$


where $\hat{R}_{t}(\alpha)$ is as in (9-29). By Lemmas 8.2 and 9.1, together with (9-7), we have

$$
\begin{aligned}
\int_{\partial \Omega_{i, \alpha}}\left(\frac{1}{2} X^{\alpha}(v)\left|\nabla u_{\alpha}\right|^{2}-\right. & \left.\left\langle X^{\alpha}\left(\nabla u_{\alpha}\right), \partial_{\nu} u_{\alpha}\right\rangle_{\mathbb{R}^{p}}\right) d \sigma_{g} \\
= & \left(n^{n-2}(n-2)^{n-1} \omega_{n-1}\left\langle\Lambda_{i},\left(Y\left(\nabla \mathcal{H}_{i}\right)\right)_{0}\right\rangle_{\mathbb{R}^{p}}+o(1)\right) \mu_{i, \alpha}^{n-2} r_{i, \alpha}^{1-n}+\hat{W}_{\alpha},
\end{aligned}
$$

where $\left(Y\left(\nabla \mathscr{H}_{i}\right)\right)_{0} \in \mathbb{R}^{p}$ is such that $\left(Y\left(\nabla \mathscr{H}_{i}\right)\right)_{0}^{l}=\sum_{\kappa=1}^{n} Y_{\kappa}\left(\nabla_{\kappa} \mathscr{H}_{i, l}\right)(0)$ for all $l=1, \ldots, p$, and

$$
\hat{W}_{\alpha}= \begin{cases}o\left(\mu_{i, \alpha}^{2}\left(-\ln \mu_{i, \alpha}\right)^{3 / 2}\right) & \text { if } n=4 \\ o\left(\mu_{i, \alpha}^{2}\right) & \text { if } n \geq 5\end{cases}
$$

As a consequence of Step 2 we have

$$
r_{i, \alpha}= \begin{cases}O\left(\left(-\ln \mu_{i, \alpha}\right)^{-1 / 2}\right) & \text { if } n=4 \\ O\left(\mu_{i, \alpha}^{(n-4) /(n-2)}\right) & \text { if } n \geq 5\end{cases}
$$

Coming back to (9-31)-(9-32), it follows that $\left\langle\Lambda_{i},\left(Y\left(\nabla \mathscr{H}_{i}\right)\right)_{0}\right\rangle_{\mathbb{R}^{p}}=0$, and since $Y$ is arbitrary, we get $\left\langle\Lambda_{i}, \nabla \mathscr{H}_{i}(0)\right\rangle_{\mathbb{R}^{p}} \equiv 0$.

\section{Proof of Theorem 0.1}

We prove Theorem 0.1 using Proposition 9.2. We let $\left(A_{\alpha}\right)_{\alpha}$ be a sequence of $C^{1}$ maps from $M$ to $M_{p}^{s}(\mathbb{R})$ such that $A_{\alpha} \rightarrow A$ in $C^{1}(M)$ as $\alpha \rightarrow+\infty$ for some $C^{1}$ map $A$ from $M$ to $M_{p}^{s}(\mathbb{R})$ satisfying $(\mathrm{H})$ and $\left(\mathrm{H}^{\prime}\right)$. We also let $\left(\mho_{\alpha}\right)_{\alpha}$ be an arbitrary bounded sequence in $H^{1}(M)$ of nonnegative solutions of (1-1) and we assume by contradiction that $\left\|u_{\alpha}\right\|_{\infty} \rightarrow+\infty$ as $\alpha \rightarrow+\infty$. We order the blow-up points of the $u_{\alpha}$ 's in such a way that

$$
\mu_{\alpha}=\mu_{1, \alpha} \geq \cdots \geq \mu_{N, \alpha},
$$

where the $\mu_{i, \alpha}$ 's are the weights of the vector bubble $\left(\mathscr{B}_{\alpha}^{i}\right)_{\alpha}$ in Proposition 4.2, and we let $\mathscr{A}_{i}$ be as in (8-4). We consider $\mathscr{A}_{1}$. By $\left(\mathrm{H}^{\prime}\right), \operatorname{Ker} A_{n}(x) \cap \operatorname{Vect}_{+}\left(\mathbb{R}^{p}\right)=\{0\}$ for all $x \in M$, where $A_{n}$ is as in $(0-2)$. In particular, if the $r_{i, \alpha}$ 's are as in (8-3), it follows from Step 2 in Section 9 that $r_{1, \alpha} \rightarrow 0$ as $\alpha \rightarrow+\infty$. As a direct consequence, $\mathscr{A}_{1} \neq \varnothing$. Let $i \in \mathscr{A}_{1}$. Still by Step 2 in Section 9, we have $r_{i, \alpha} \rightarrow 0$ as $\alpha \rightarrow+\infty$. By Proposition 9.2, since $\operatorname{Ker} A_{n}(x) \cap \operatorname{Vect}_{+}\left(\mathbb{R}^{p}\right)=\{0\}$ for all $x \in M$, for any $i \in \mathscr{A}_{1} \cup\{1\}$, there exists $C_{i}>0$ such that

$$
r_{i, \alpha}^{2} \ln \frac{1}{\mu_{i, \alpha}} \rightarrow C_{i} \text { if } n=4 \quad \text { and } \quad r_{i, \alpha}^{n-2} \mu_{i, \alpha}^{4-n} \rightarrow C_{i} \text { if } n \geq 5
$$

as $\alpha \rightarrow+\infty$. By (10-1), $\mu_{i, \alpha}=o\left(r_{i, \alpha}\right)$ for all $i \in \mathscr{A}_{1} \cup\{1\}$. We also get from (10-1) that for any $i \in \mathscr{A}_{1} \cup\{1\}$,

$$
\mu_{i, \alpha}=o\left(r_{i, \alpha}^{2}\right) \text { if } n=4,5 \quad \text { and } \quad r_{i, \alpha}^{2}=o\left(\mu_{i, \alpha}\right) \text { if } n \geq 7 .
$$

As a remark, it follows from (10-2) that $u_{\infty} \equiv 0$ when $n=4,5$ since, if not the case, $r_{i, \alpha}^{2} \leq \mu_{i, \alpha}$. It also follows from (10-2) that for any $i \in \mathscr{A}_{1} \cup\{1\}, \mathscr{A}_{i} \cap \mathscr{B}_{i} \neq \varnothing$, where the $\mathscr{B}_{i}$ 's are as in (8-8). By (9-3), we get with (10-2) that

$$
\mathscr{H}_{i}(z)=\sum_{j \in \mathscr{A}_{i} \cap \mathscr{B}_{i}} \frac{\lambda_{i, j} \Lambda_{j}}{\left|z-z_{i, j}\right|^{n-2}}
$$


where $\mathscr{H}_{i}$ is as in Lemma 9.1. In particular, the $\Lambda_{i}$ 's are the $S^{p-1}$ projections of the bubbles $\left(\mathscr{P}_{\alpha}^{i}\right)_{\alpha}$. Let $\mathscr{E}_{1}=\left(\mathscr{A}_{1} \cap \mathscr{B}_{1}\right) \cup\{1\}$. For any $i \in \mathscr{A}_{1} \cap \mathscr{B}_{1}$, we have $\mathscr{A}_{i} \cap \mathscr{B}_{i}=\mathscr{E}_{1} \backslash\{i\}$. We pick up some $i \in \mathscr{E}_{1}$ such that $d_{g}\left(x_{1, \alpha}, x_{i, \alpha}\right) \geq d_{g}\left(x_{1, \alpha}, x_{j, \alpha}\right)$ for all $j \in \mathscr{E}_{1}$. By Proposition 9.2 we have $\left\langle\Lambda_{i}, \nabla \mathscr{H}_{i}(0)\right\rangle_{\mathbb{R}^{p}}=0$. Together with (10-3), this implies that $\left\langle\Lambda_{i}, \Lambda_{j}\right\rangle_{\mathbb{R}^{p}}=0$ for all $j \in \mathscr{E}_{1} \backslash\{i\}$. Repeating the operation with $\mathscr{E}_{1} \backslash\{i\}$, and so on up to exhaust all the indices in $\mathscr{E}_{1}$, we obtain that $\left\langle\Lambda_{i}, \Lambda_{j}\right\rangle_{\mathbb{R}^{p}}=\delta_{i j}$ for all $i, j \in \mathscr{E}_{1}$. Moreover, it follows from (9-4) and (9-5) in Proposition 9.2 that $V=\operatorname{Vect}\left\{\Lambda_{i}, i \in \mathscr{E}_{1}\right\}$ is a stable vector space of $A_{n}\left(x_{1}\right)$. Noting that $\left\langle\Lambda_{i}, \mathscr{H}_{i}(0)\right\rangle_{\mathbb{R}^{p}}=0$ for all $i \in \mathscr{E}_{1}$, we also get with (9-4) and (9-5) in Proposition 9.2 that the $\Lambda_{i}$ 's are isotropic vectors for $A_{n}\left(x_{1}\right)$ for all $i \in \mathscr{E}_{1}$. In particular, we get a contradiction with $\left(\mathrm{H}^{\prime}\right)$. This proves Theorem 0.1 when $n \neq 6$. When $n=6$, thanks to Proposition 2.1 , it remains to prove that our systems are weakly stable, and thus that we necessarily have $u_{\infty} \not \equiv 0$ if we assume $\left(\mathrm{H}^{\prime}\right)$. When $n=6$, it follows from (10-1) that $r_{i, \alpha}^{2} \sim \mu_{i, \alpha}$. Then, by (9-3),

$$
\mathscr{H}_{i}(z)=\sum_{j \in \mathscr{A}_{i} \cap \Re_{i}} \frac{\lambda_{i, j} \Lambda_{j}}{\left|z-z_{i, j}\right|^{n-2}}+C \boldsymbol{u}_{\infty}\left(x_{1}\right),
$$

where $r_{i, \alpha}^{-4} \mu_{i, \alpha}^{2} \rightarrow C$ as $\alpha \rightarrow+\infty$. As above, $\left\langle\Lambda_{i}, \Lambda_{j}\right\rangle_{\mathbb{R}^{p}}=\delta_{i j}$ for all $i, j \in \mathscr{E}_{1}$, but we may have $\mathscr{E}_{1}=\{1\}$. By Proposition 9.2, $V=\operatorname{Vect}\left\{\Lambda_{i}, i \in \mathscr{E}_{1}\right\}$ is a stable vector space of $A_{6}\left(x_{1}\right)$ and the $\Lambda_{i}$ 's are isotropic vectors for $A_{6}\left(x_{1}\right)$ for all $i \in \mathscr{E}_{1}$ if $\mho_{\infty}\left(x_{1}\right)=0$. In particular, we do get a contradiction with $\left(\mathrm{H}^{\prime}\right)$ if $u_{\infty}\left(x_{1}\right)=0$. This proves Theorem 0.1 when $n=6$.

As a remark, if $n=6$ and $A_{6}<0$ in $M$ in the sense of bilinear forms, where $A_{6}$ is as in (0-2), then we also get a contradiction by (9-5) in Proposition 9.2 since $r_{i, \alpha}^{2} \sim \mu_{i, \alpha}$ and $\mathscr{H}_{i}(0),\left\langle\Lambda_{i}, \mathscr{H}_{i}(0)\right\rangle_{\mathbb{R}^{p}} \Lambda_{i} \in$ $\operatorname{Vect}_{+}\left(\mathbb{R}^{p}\right)$. In particular, we recover analytic stability for our systems if we assume that $A_{6}<0$ in $M$ in the sense of bilinear forms. More precisely, letting $(M, g)$ be a smooth compact six-dimensional Riemannian manifold, $p \geq 1$ be an integer, and $A: M \rightarrow M_{p}^{s}(\mathbb{R})$ be a $C^{1}$-map such that $A$ satisfies $(\mathrm{H})$, the system (0-1) associated with $A$ is analytically stable if $A_{6}(x)<0$ in the sense of bilinear forms for all $x$.

As another remark, it is easily seen from (9-4) and (9-5) in Proposition 9.2 that for any $n \geq 4$, and any $i \in \mathscr{A}_{1} \cup\{1\}, A_{n}\left(x_{1}\right) \Lambda_{i} \in \operatorname{Vect}_{+}\left(\mathbb{R}^{p}\right)$. In particular, we can replace $\left(\mathrm{H}^{\prime}\right)$ in Theorem 0.1 by the slightly more general condition that for any $x \in M$, and any $k \in\{1, \ldots, p\}$, there does not exist an orthonormal family $\left(e_{1}, \ldots, e_{k}\right)$ of vectors in $\operatorname{Is}_{A_{n}(x)} \cap \operatorname{Vect}_{+}\left(\mathbb{R}^{p}\right)$ such that $A_{n}(x) V \subset V$ and $A_{n}(x) e_{i} \in \operatorname{Vect}_{+}\left(\mathbb{R}^{p}\right)$ for all $i$, where $V$ is the $k$-dimensional subspace of $\mathbb{R}^{p}$ with basis $\left(e_{1}, \ldots, e_{k}\right)$.

As a final remark we mention that Theorem 0.1 still holds true, and can be proved with only slight modifications in the arguments of Section 9 , if the $C^{1}$ convergence of the $A_{\alpha}$ 's is replaced by a $C^{0, \theta_{-}}$ convergence of the $A_{\alpha}$ 's with $\theta=1$ when $n=4$, and $\theta>2 /(n-2)$ when $n \geq 5$.

\section{References}

[Ablowitz et al. 2004] M. J. Ablowitz, B. Prinari, and A. D. Trubatch, Discrete and continuous nonlinear Schrödinger systems, London Mathematical Society Lecture Note Series 302, Cambridge University Press, 2004. MR 2005c:37117 Zbl 1057.35058

[Akhmediev and Ankiewicz 1998] N. Akhmediev and A. Ankiewicz, "Partially coherent solitons on a finite background", Phys. Rev. Lett. 82 (1998), 2661-2664.

[Aubin 1976] T. Aubin, "Équations différentielles non linéaires et problème de Yamabe concernant la courbure scalaire", $J$. Math. Pures Appl. (9) 55:3 (1976), 269-296. MR 55 \#4288 Zbl 0336.53033 
[Brendle 2008a] S. Brendle, "Blow-up phenomena for the Yamabe equation", J. Amer. Math. Soc. 21:4 (2008), 951-979. MR 2425176

[Brendle 2008b] S. Brendle, "On the conformal scalar curvature equation and related problems", pp. 1-19 in Surveys in differential geometry, XII: Geometric flows, edited by H.-D. Cao and S.-T. Yau, Int. Press, Somerville, MA, 2008. MR 2488950 Zbl 05504035

[Brendle and Marques 2009] S. Brendle and F. C. Marques, "Blow-up phenomena for the Yamabe equation. II", J. Differential Geom. 81:2 (2009), 225-250. MR 2472174 Zbl 1166.53025

[Burke et al. 1997] J. Burke, J. Bohn, B. Esry, and C. Greene, "Hartree-Fock theory for double condensates", Phys. Rev. Lett. 78 (1997), 3594-3597.

[Caffarelli et al. 1989] L. A. Caffarelli, B. Gidas, and J. Spruck, "Asymptotic symmetry and local behavior of semilinear elliptic equations with critical Sobolev growth", Comm. Pure Appl. Math. 42:3 (1989), 271-297. MR 90c:35075 Zbl 0702.35085

[Christodoulides et al. 1997] D. Christodoulides, T. Coskun, M. Mitchell, and M. Segev, "Theory of incoherent self-focusing in biased photorefractive media", Phys. Rev. Lett. 78 (1997), 646-649.

[Druet 2003] O. Druet, "From one bubble to several bubbles: the low-dimensional case", J. Differential Geom. 63:3 (2003), 399-473. MR 2004h:53051 Zbl 1070.53017

[Druet 2004] O. Druet, "Compactness for Yamabe metrics in low dimensions", Int. Math. Res. Not. 23 (2004), 1143-1191. MR 2005b:53056 Zbl 1085.53029

[Druet and Hebey 2005a] O. Druet and E. Hebey, "Blow-up examples for second order elliptic PDEs of critical Sobolev growth”, Trans. Amer. Math. Soc. 357:5 (2005), 1915-1929. MR 2005i:58023 Zbl 1061.58017

[Druet and Hebey 2005b] O. Druet and E. Hebey, "Elliptic equations of Yamabe type", IMRS Int. Math. Res. Surv. 1 (2005), 1-113. MR 2006b:53046 Zbl 1081.53034

[Druet et al. 2004] O. Druet, E. Hebey, and F. Robert, Blow-up theory for elliptic PDEs in Riemannian geometry, Mathematical Notes 45, Princeton University Press, 2004. MR 2005g:53058 Zbl 1059.58017

[Druet et al. 2009] O. Druet, E. Hebey, and J. Vétois, "Bounded stability for strongly coupled critical elliptic systems below the geometric threshold of the conformal laplacian", 2009. To appear in J. Funct. Anal.

[Gidas and Spruck 1981] B. Gidas and J. Spruck, "A priori bounds for positive solutions of nonlinear elliptic equations", Comm. Partial Differential Equations 6:8 (1981), 883-901. MR 82h:35033 Zbl 0462.35041

[Hebey and Vaugon 2001] E. Hebey and M. Vaugon, "From best constants to critical functions", Math. Z. 237:4 (2001), 737767. MR 2002h:58061 Zbl 0992.58016

[Hioe 1999] F. Hioe, "Solitary waves for $N$ coupled nonlinear Schrödinger equations", Phys. Rev. Lett. 82 (1999), 1152-1155.

[Hioe and Salter 2002] F. T. Hioe and T. S. Salter, "Special set and solutions of coupled nonlinear Schrödinger equations", $J$. Phys. A 35:42 (2002), 8913-8928. MR 2003k:35230 Zbl 1040.35115

[Kanna and Lakshmanan 2001] T. Kanna and M. Lakshmanan, "Exact soliton solutions, shape changing collisions, and partially coherent solitons in coupled nonlinear Schrödinger equations", Phys. Rev. Lett. 86 (2001), 5043-5046.

[Khuri et al. 2009] M. A. Khuri, F. C. Marques, and R. M. Schoen, "A compactness theorem for the Yamabe problem", J. Differential Geom. 81:1 (2009), 143-196. MR 2477893 Zbl 1162.53029

[Li and Zhang 2004] Y. Y. Li and L. Zhang, "A Harnack type inequality for the Yamabe equation in low dimensions”, Calc. Var. Partial Differential Equations 20:2 (2004), 133-151. MR 2005f:53053 Zbl 1113.35070

[Li and Zhang 2005] Y. Y. Li and L. Zhang, "Compactness of solutions to the Yamabe problem, II", Calc. Var. Partial Differential Equations 24:2 (2005), 185-237. MR 2006f:53049 Zbl 02220860

[Li and Zhu 1999] Y. Li and M. Zhu, "Yamabe type equations on three-dimensional Riemannian manifolds", Commun. Contemp. Math. 1:1 (1999), 1-50. MR 2000m:53051 Zbl 0973.53029

[Marques 2005] F. C. Marques, "A priori estimates for the Yamabe problem in the non-locally conformally flat case", $J$. Differential Geom. 71:2 (2005), 315-346. MR 2006i:53046 Zbl 1101.53019

[Schoen 1989] R. M. Schoen, "Variational theory for the total scalar curvature functional for Riemannian metrics and related topics", pp. 120-154 in Topics in calculus of variations (Montecatini Terme, 1987), edited by M. Giaquinta, Lecture Notes in Math. 1365, Springer, Berlin, 1989. MR 90g:58023 Zbl 0702.49038 
[Schoen 1991] R. M. Schoen, "On the number of constant scalar curvature metrics in a conformal class", pp. 311-320 in Differential geometry, edited by B. Lawson and K. Tenenblat, Pitman Monogr. Surveys Pure Appl. Math. 52, Longman Sci. Tech., Harlow, 1991. MR 94e:53035 Zbl 0733.53021

[Struwe 1984] M. Struwe, "A global compactness result for elliptic boundary value problems involving limiting nonlinearities", Math. Z. 187:4 (1984), 511-517. MR 86k:35046 Zbl 0535.35025

[Struwe 1990] M. Struwe, Variational methods, Springer, Berlin, 1990. MR 92b:49002 Zbl 0746.49010

[Trudinger 1968] N. S. Trudinger, "Remarks concerning the conformal deformation of Riemannian structures on compact manifolds”, Ann. Scuola Norm. Sup. Pisa (3) 22 (1968), 265-274. MR 39 \#2093 Zbl 0159.23801

Received 29 Jan 2009. Revised 22 Jun 2009. Accepted 21 Jul 2009.

OLIVIER DRUET: Olivier.Druet@umpa.ens-lyon.fr

Ecole normale supérieure de Lyon, Département de Mathématiques - UMPA, 46 allée d'Italie, 69364 Lyon Cedex 07, France

EMmANUEL HeBeY: hebey@u-cergy .fr

Université de Cergy-Pontoise, Département de Mathématiques, Site de Saint Martin, 2 avenue Adolphe Chauvin, 95302 Cergy-Pontoise Cedex, France 


\title{
Analysis \& PDE
}

pjm.math.berkeley.edu/apde

EDITORS

\author{
EDITOR-IN-CHIEF \\ Maciej Zworski \\ University of California \\ Berkeley, USA
}

BOARD OF EDITORS

\begin{tabular}{|c|c|c|c|}
\hline Michael Aizenman & $\begin{array}{l}\text { Princeton University, USA } \\
\text { aizenman@math.princeton.edu }\end{array}$ & Nicolas Burq & $\begin{array}{l}\text { Université Paris-Sud 11, France } \\
\text { nicolas.burq @math.u-psud.fr }\end{array}$ \\
\hline Luis A. Caffarelli & $\begin{array}{l}\text { University of Texas, USA } \\
\text { caffarel@math.utexas.edu }\end{array}$ & un-Yung Alice Chang & $\begin{array}{l}\text { Princeton University, USA } \\
\text { chang@math.princeton.edu }\end{array}$ \\
\hline Michael Christ & $\begin{array}{l}\text { University of California, Berkeley, USA } \\
\text { mchrist@math.berkeley.edu }\end{array}$ & Charles Fefferman & $\begin{array}{l}\text { Princeton University, USA } \\
\text { cf@math.princeton.edu }\end{array}$ \\
\hline Ursula Hamenstaedt & $\begin{array}{l}\text { Universität Bonn, Germany } \\
\text { ursula@math.uni-bonn.de }\end{array}$ & Nigel Higson & $\begin{array}{l}\text { Pennsylvania State Univesity, USA } \\
\text { higson@ math.psu.edu }\end{array}$ \\
\hline Vaughan Jones & $\begin{array}{l}\text { University of California, Berkeley, USA } \\
\text { vfr@math.berkeley.edu }\end{array}$ & Herbert Koch & $\begin{array}{l}\text { Universität Bonn, Germany } \\
\text { koch@math.uni-bonn.de }\end{array}$ \\
\hline Izabella Laba & $\begin{array}{l}\text { University of British Columbia, Canada } \\
\text { ilaba@math.ubc.ca }\end{array}$ & Gilles Lebeau & $\begin{array}{l}\text { Université de Nice Sophia Antipolis, France } \\
\text { lebeau@unice.fr }\end{array}$ \\
\hline László Lempert & $\begin{array}{l}\text { Purdue University, USA } \\
\text { lempert@math.purdue.edu }\end{array}$ & Richard B. Melrose & $\begin{array}{l}\text { Massachussets Institute of Technology, USA } \\
\text { rbm@math.mit.edu }\end{array}$ \\
\hline Frank Merle & $\begin{array}{l}\text { Université de Cergy-Pontoise, France } \\
\text { Frank.Merle@u-cergy.fr }\end{array}$ & William Minicozzi II & $\begin{array}{l}\text { Johns Hopkins University, USA } \\
\text { minicozz@ math.jhu.edu }\end{array}$ \\
\hline Werner Müller & $\begin{array}{l}\text { Universität Bonn, Germany } \\
\text { mueller@math.uni-bonn.de }\end{array}$ & Yuval Peres & $\begin{array}{l}\text { University of California, Berkeley, USA } \\
\text { peres@stat.berkeley.edu }\end{array}$ \\
\hline Gilles Pisier & $\begin{array}{l}\text { Texas A\&M University, and Paris } 6 \\
\text { pisier@math.tamu.edu }\end{array}$ & Tristan Rivière & $\begin{array}{l}\text { ETH, Switzerland } \\
\text { riviere@math.ethz.ch }\end{array}$ \\
\hline Igor Rodnianski & $\begin{array}{l}\text { Princeton University, USA } \\
\text { irod@ math.princeton.edu }\end{array}$ & Wilhelm Schlag & $\begin{array}{l}\text { University of Chicago, USA } \\
\text { schlag@math.uchicago.edu }\end{array}$ \\
\hline Sylvia Serfaty & $\begin{array}{l}\text { New York University, USA } \\
\text { serfaty@ cims.nyu.edu }\end{array}$ & Yum-Tong Siu & $\begin{array}{l}\text { Harvard University, USA } \\
\text { siu@math.harvard.edu }\end{array}$ \\
\hline Terence Tao & $\begin{array}{l}\text { University of California, Los Angeles, USA } \\
\text { tao@math.ucla.edu }\end{array}$ & A Michael E. Taylor & $\begin{array}{l}\text { Univ. of North Carolina, Chapel Hill, USA } \\
\text { met@math.unc.edu }\end{array}$ \\
\hline Gunther Uhlmann & $\begin{array}{l}\text { University of Washington, USA } \\
\text { gunther@math.washington.edu }\end{array}$ & András Vasy & $\begin{array}{l}\text { Stanford University, USA } \\
\text { andras@math.stanford.edu }\end{array}$ \\
\hline Virgil Voiculescu & $\begin{array}{l}\text { University of California, Berkeley, USA } \\
\text { dvv@math.berkeley.edu }\end{array}$ & Steven Zelditch & $\begin{array}{l}\text { Johns Hopkins University, USA } \\
\text { szelditch@math.jhu.edu }\end{array}$ \\
\hline
\end{tabular}

\section{PRODUCTION}

apde@mathscipub.org

Paulo Ney de Souza, Production Manager Sheila Newbery, Production Edito

Silvio Levy, Senior Production Editor

See inside back cover or pjm.math.berkeley.edu/apde for submission instructions.

The subscription price for 2009 is US \$120/year for the electronic version, and \$180/year for print and electronic. Subscriptions, requests for back issues from the last three years and changes of subscribers address should be sent to Mathematical Sciences Publishers, Department of Mathematics, University of California, Berkeley, CA 94720-3840, USA.

Analysis \& PDE, at Mathematical Sciences Publishers, Department of Mathematics, University of California, Berkeley, CA 94720-3840 is published continuously online. Periodical rate postage paid at Berkeley, CA 94704, and additional mailing offices.

APDE peer-review and production is managed by EditFLOW ${ }^{\mathrm{TM}}$ from Mathematical Sciences Publishers.

PUBLISHED BY

mathematical sciences publishers

http://www.mathscipub.org

A NON-PROFIT CORPORATION

Typeset in IATEX

Copyright (C2009 by Mathematical Sciences Publishers 


\section{ANALYSIS \& PDE}

\section{Volume $2 \quad$ No. $3 \quad 2009$}

Global existence of smooth solutions of a 3D log-log energy-supercritical wave equation

TRISTAN ROY

Periodic stochastic Korteweg-de Vries equation with additive space-time white noise

TADAHIRO OH

Stability for strongly coupled critical elliptic systems in a fully inhomogeneous medium

305

OLIVIER DRUET and EMMANUEL HEBEY

Global regularity for a logarithmically supercritical hyperdissipative Navier-Stokes equation 361

TERENCE TAO 